\title{
Analytic families of quantum hyperbolic invariants
}

\author{
STÉPHANE BASEILHAC \\ RICCARDO BENEDETTI
}

\begin{abstract}
We organize the quantum hyperbolic invariants (QHI) of 3-manifolds into sequences of rational functions indexed by the odd integers $N \geq 3$ and defined on moduli spaces of geometric structures refining the character varieties. In the case of onecusped hyperbolic 3-manifolds $M$ we generalize the $\mathrm{QHI}$ and get rational functions $\mathcal{H}_{N}^{h_{f}, h_{c}, k_{c}}$ depending on a finite set of cohomological data $\left(h_{f}, h_{c}, k_{c}\right)$ called weights. These functions are regular on a determined Abelian covering of degree $N^{2}$ of a Zariski open subset, canonically associated to $M$, of the geometric component of the variety of augmented $\operatorname{PSL}(2, \mathbb{C})$-characters of $M$. New combinatorial ingredients are a weak version of branchings which exists on every triangulation, and state sums over weakly branched triangulations, including a sign correction which eventually fixes the sign ambiguity of the QHI. We describe in detail the invariants of three cusped manifolds, and present the results of numerical computations showing that the functions $\mathcal{H}_{N}^{h_{f}, h_{c}, k_{c}}$ depend on the weights as $N \rightarrow \infty$, and recover the volume for some specific choices of the weights.
\end{abstract}

57M27, 57Q15; 57R56

\section{Introduction}

In the series of papers $[3 ; 4 ; 5]$, we defined a family of complex-valued quantum invariants $\mathcal{H}_{N}(\mathcal{P})$ of patterns $\mathcal{P}$ of geometric nature, called quantum hyperbolic invariants $(\mathrm{QHI})$ and indexed by the odd integers $N \geq 3$. Roughly, a pattern consists of a compact oriented 3-manifold equipped with a representation of the fundamental group in $\operatorname{PSL}(2, \mathbb{C})$, plus some compatible cohomological data (see the next subsections for a complete definition). The QHI generalize the Kashaev invariants of links in $S^{3}$ [6], subject to the celebrated volume conjecture. They define a $(2+1)$-dimensional quantum field theory and, when applied to mapping cylinders of surface diffeomorphisms, they coincide with the invariants derived from the local version of finite-dimensional quantum Teichmüller theory (see Bai [1], Bai, Bonahon and Liu [2], and Baseilhac and Benedetti [5]). 
In $[3 ; 4 ; 5]$, we prove the invariance of the QHI only up to sign and multiplication by $N^{\text {th }}$ roots of unity. The eventual existence and the meaning of such a phase anomaly, as well as the determination of the asymptotic behavior of $\mathcal{H}_{N}(\mathcal{P})$ as $N$ approaches $+\infty$, are two main open issues of the theory. In order to tackle them, it seemed necessary to develop a functional approach that would clarify the intrinsic nature of the various combinatorial and geometric ingredients involved in the definition of the QHI. To this end, we achieve the following goals in this paper.

QHI of cusped manifolds We extend the QHI of any one-cusped hyperbolic 3manifold $M$ to invariants defined on the geometric component $X_{0}(M)$ of the variety of augmented PSL $(2, \mathbb{C})$-characters of $M$. In [4; 5], the QHI of cusped hyperbolic manifolds were defined only at the hyperbolic holonomy.

State sums over weakly branched triangulations To achieve the previous goal it is necessary to introduce state sums over triangulations that do not support any branching, replaced by relaxed structures called weak branchings. These state sums give rise to QHI generalizing all the previously defined ones.

Analytic families of QHI We recast all the QHI into sequences $\left\{\mathcal{A}_{N}(Y)\right\}$ of families of complex analytic spaces and maps, indexed by the odd integers $N \geq 3$. Each family $\mathcal{A}_{N}(Y)$ is associated to a topological support $Y$, and provides concrete models of geometric structures over $Y$ called patterns. Patterns over cusped manifolds have an intrinsic meaning in terms of the $\operatorname{PSL}(2, \mathbb{C})$-version of the $A$-polynomial, and natural relationships with Chern-Simons theory.

Fixing the sign ambiguity We include a sign correction in the state sum formulas of the QHI, which removes their sign ambiguity under a mild assumption on the bulk $c$-weight; see below. The sign correction depends on the combinatorics of the weak branching and is a byproduct of Bendetti and Petronio [9]. It becomes trivial when dealing (when possible) with branched triangulations, so that the QHI of $[3 ; 4 ; 5]$ are eventually defined up to multiplication by $N^{\text {th }}$ roots of unity.

In a sequel to this paper (in collaboration with C Frohman), we will develop our approach concerning the asymptotic behavior of the QHI. Basically, given a topological support $Y$ and a sequence $\left\{\mathcal{P}_{N}\right\}$ of patterns over $Y$, we study the limit of the family $\left\{\mathcal{A}_{N}(Y)\right\}$ as $N$ approaches $+\infty$, instead of $\left\{\mathcal{H}_{N}\left(\mathcal{P}_{N}\right)\right\}$ for a single sequence $\left\{\mathcal{P}_{N}\right\}$. Then, assuming that

$$
\mathcal{H}_{\infty}\left(\left\{\mathcal{P}_{N}\right\}\right):=\limsup _{N \rightarrow \infty}\left(\log \left|\mathcal{H}_{N}\left(\mathcal{P}_{N}\right)\right| / N\right)
$$

is finite (this is the case for all natural sequences $\left\{\mathcal{P}_{N}\right\}$ ), we consider the following problems: 
(1) Determine the nature (regularity) of $\mathcal{H}_{\infty}\left(\left\{\mathcal{P}_{N}\right\}\right)$ as a function of the patterns over $Y$.

(2) Describe the asymptotic behavior of $\left\{\mathcal{H}_{N}\left(\mathcal{P}_{N}\right)\right\}$ as $N$ approaches $+\infty$ in terms of classical geometric invariants of $Y$ : Chern-Simons invariants, torsions, twisted cohomology etc.

The Kashaev-Murakami-Murakami volume conjecture is a particular case of (2), for constant sequences of patterns associated to links in $S^{3}$ (see Theorem 1.2 below).

In the rest of this introduction we describe with more details the content of the paper.

\section{A QHI of cusped manifold patterns}

In this paper we define a cusped manifold to be an oriented, connected, noncompact complete hyperbolic 3-manifold of finite volume with exactly one cusp. Hence a cusped manifold $M$ is diffeomorphic to the interior of a compact 3-manifold denoted by $V$, with one torus boundary component.

A pattern $\mathcal{P}=\left(Y_{\mathcal{P}}, \rho,(h, k)\right)$ over $M$ consists of a topological support $Y_{\mathcal{P}}$ together with additional geometric structures determined by the couple $(\rho,(h, k))$. The topological support takes the form $Y_{\mathcal{P}}=\left(V,\left(h_{c}, k_{c}\right)\right)$, where

$$
\left(h_{c}, k_{c}\right) \in H^{1}(V ; \mathbb{Z} / 2 \mathbb{Z}) \times H^{1}(\partial V ; \mathbb{Z})
$$

is a so-called $c$-weight, defined by a bulk $c$-weight $h_{c}$ and a boundary $c$-weight $k_{c}$ satisfying

$$
r\left(k_{c}\right)=\iota^{*}\left(h_{c}\right),
$$

where $r: H^{1}(\partial V ; \mathbb{Z}) \rightarrow H^{1}(\partial V ; \mathbb{Z} / 2 \mathbb{Z})$ is the reduction $\bmod 2$, and the function $\iota^{*}: H^{1}(V ; \mathbb{Z} / 2 \mathbb{Z}) \rightarrow H^{1}(\partial V ; \mathbb{Z} / 2 \mathbb{Z})$ is induced by the inclusion map $\iota: \partial V \rightarrow V$. The pattern $\mathcal{P}$ is obtained by completing $Y_{\mathcal{P}}$ with a couple $\left(\rho,\left(h_{f}, k_{f}\right)\right)$, where $\rho$ is a $\operatorname{PSL}(2, \mathbb{C})$-character of $V$, ie a conjugacy class of representations of $\pi_{1}(V)$ in $\operatorname{PSL}(2, \mathbb{C})$, and $\left(h_{f}, k_{f}\right) \in H^{1}(V ; \mathbb{Z} / 2 \mathbb{Z}) \times H^{1}(\partial V ; \mathbb{C})$ is a so-called $f$-weight (relative to $\rho$ ), defined by a bulk $f$-weight $h_{f}$ and a boundary $f$-weight $k_{f}$ satisfying the following constraint. Up to conjugacy the restriction of $\rho$ to the torus $\partial V$ is valued in the group of complex affine transformations of the plane; the linear part of this restriction defines a class in $H^{1}\left(\partial V ; \mathbb{C}^{*}\right)$. Let $d \in H^{1}(\partial V ; \mathbb{C} / 2 i \pi \mathbb{Z})$ be the log of this class, with imaginary part in $]-\pi, \pi]$. One requires that for all $a \in H_{1}(\partial V ; \mathbb{Z})$,

$$
k_{f}(a)=d(a) \quad \bmod i \pi \quad \text { and } \quad\left(k_{f}(a)-d(a)\right) / i \pi=\imath^{*}\left(h_{f}\right)(a) \quad \bmod 2 .
$$

Collecting the bulk and boundary weights we will often write $\mathcal{P}$ as $(V, \rho,(h, k))$, where

$$
(h, k)=\left(\left(h_{f}, h_{c}\right),\left(k_{f}, k_{c}\right)\right) .
$$


Notation For every $n \in \mathbb{N}$, we write $a \equiv_{n} b$ to mean that $a$ and $b$ are equal up to multiplication by a power of $\exp (2 i \pi / n)$. If $n$ is odd, then $a \equiv_{2 n} b$ if and only if $a \equiv_{n} \pm b$. We denote $\mu_{n}$ the group of $n^{\text {th }}$ roots of unity, acting on $\mathbb{C}$ by multiplication.

In [4; 5], for every cusped manifold $M$ and odd $N \geq 3$, we defined quantum hyperbolic invariants

$$
\mathcal{H}_{N}(M):=\mathcal{H}_{N}\left(V, \rho_{\text {hyp }},(0,0)\right) \in \mathbb{C} / \mu_{2 N},
$$

that is, for the pattern $\mathcal{P}=\left(V, \rho_{\text {hyp }},(0,0)\right)$, where $\rho_{\text {hyp }}$ is the hyperbolic holonomy of $M$ and all weights vanish. The following theorem summarizes our new results for patterns over $M$ (all terms are defined in Section 4).

Theorem 1.1 Let $M$ be an arbitrary cusped manifold, $X(M)$ the variety of augmented PSL $(2, \mathbb{C})$-characters of $M$, and $X_{0}(M) \subset X(M)$ the irreducible component of $\rho_{\text {hyp }}$. There is a canonical nonempty Zariski open subset $\Omega(M)$ of $X_{0}(M)$ containing $\rho_{\text {hyp }}$ such that:

(1) For every odd integer $N \geq 3$ and every pattern $(V, \rho,(h, k))$ such that $\rho \in \Omega(M)$, there is a quantum hyperbolic invariant $\mathcal{H}_{N}(V, \rho,(h, k)) \in \mathbb{C} / \mu_{2 N}$ satisfying $\mathcal{H}_{N}\left(V, \rho_{\text {hyp }},(0,0)\right)=\mathcal{H}_{N}(M)$.

(2) (Analytic families) Fix a topological support $\left(V,\left(h_{c}, k_{c}\right)\right)$ and an element $h_{f}$ in $H^{1}(V ; \mathbb{Z} / 2 \mathbb{Z})$. For every odd integer $N \geq 3$, the invariants $\mathcal{H}_{N}(V, \rho,(h, k))$

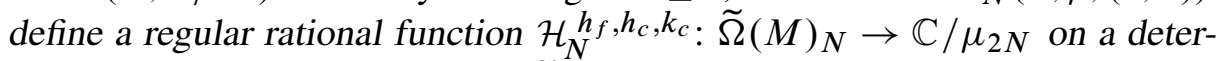
mined $(\mathbb{Z} / N \mathbb{Z})^{2}$-covering space $\widetilde{\Omega}(M)_{N}$ of $\Omega(M)$.

(3) (Resolution of the sign ambiguity) If $N \equiv 1 \bmod 4$, or $N \equiv 3 \bmod 4$ and $h_{c}=0$, then (1) and (2) above hold true when $\mu_{2 N}$ is replaced by $\mu_{N}$.

Comments (a) $X(M)$ is a complex algebraic variety and $\rho_{\text {hyp }}$ a regular point of $X(M)$. Hence there is a unique irreducible component $X_{0}(M)$ of $X(M)$ containing $\rho_{\text {hyp }}$. As $M$ has only one cusp, $X_{0}(M)$ is an algebraic curve and $\Omega(M)$ is the complement of a finite set of points.

(b) Theorem 1.1(2) is a rough qualitative formulation of the $N^{\text {th }}$ analytic family $\mathcal{A}_{N}(Y)$ associated to the topological support $Y=\left(V,\left(h_{c}, k_{c}\right)\right)$ of $M$.

(c) The arguments of Theorem 1.1(1)-(2) apply verbatim to prove the invariance of simplicial formulas defining the $\operatorname{PSL}(2, \mathbb{C})$-Chern-Simons section, realized as an analytic equivariant function $\mathfrak{h}^{*} \mathcal{H}_{1}^{\mathcal{F}_{\mathrm{EP}}, h_{f}}: \widetilde{\Omega}(M)_{\infty} \rightarrow \mathbb{C}$ on a $\mathbb{Z}^{2}$-covering $\widetilde{\Omega}(M)_{\infty}$ of $\Omega(M)$; see Section 4D. 


\section{B QHI of other patterns}

In order to explain the nature of the QHI in Theorem 1.1(1), and why this result is not straightforward, it is useful to recall a few general facts from $[3 ; 4 ; 5]$.

QHFT partition functions The QHI of quantum hyperbolic field-theoretic (QHFT) patterns have been defined in [5]. The topological supports of QHFT patterns have the form $\left(V, L,\left(h_{c}, k_{c}\right)\right)$, where:

- $\quad V$ is a compact oriented connected 3-manifold with (possibly empty) boundary $\partial V$ made by torus components; if $\partial V=\varnothing$ we will write $W$ instead of $V$.

- $L$ is a nonempty link in the interior of $V$.

- The bulk and boundary $c$-weights $\left(h_{c}, k_{c}\right) \in H^{1}(V ; \mathbb{Z} / 2 \mathbb{Z}) \times H^{1}(\partial V ; \mathbb{Z})$ satisfy (1).

The QHFT patterns are obtained by completing $\left(V, L,\left(h_{c}, k_{c}\right)\right)$ with a pair $\left(\rho,\left(h_{f}, k_{f}\right)\right)$, where $\rho$ is any $\operatorname{PSL}(2, \mathbb{C})$-character of $V$ and $\left(h_{f}, k_{f}\right)$ are bulk and boundary $f-$ weights satisfying (2) with respect to $\rho$.

When $V=W$ is a closed 3-manifold, $\left(k_{c}, k_{f}\right)$ disappears so that $h=\left(h_{c}, h_{f}\right) \in$ $H^{1}(W ; \mathbb{Z} / 2 \mathbb{Z})^{2}$. In $[3 ; 4]$ we defined the QHI $\mathcal{H}_{N}(W, L, \rho, h)$ in that situation for every character $\rho$ and weight $h$. A specialization is $H_{N}\left(S^{3}, L\right):=\mathcal{H}_{N}\left(S^{3}, L, \rho_{\text {triv }}, 0\right)$, where $\rho_{\text {triv }}$ is the trivial character of $S^{3}$. In [6] we obtained the following result, which establishes a connection with Jones invariants.

Theorem 1.2 For every link $L$ in $S^{3}$ and every odd integer $N \geq 3$,

$$
H_{N}\left(S^{3}, L\right) \equiv_{N} L L{ }_{N}=J_{N}(L)\left(e^{2 i \pi / N}\right),
$$

where $\angle L \triangle_{N}$ is the link invariant defined by the enhanced Yang-Baxter operator extending the Kashaev $R$-matrix, and $J_{N}(L)(q) \in \mathbb{Z}\left[q^{ \pm 1}\right]$ is the colored Jones polynomial, normalized so that $J_{N}\left(K_{U}\right)(q)=1$ on the unknot $K_{U}$.

Remark 1.3 The second equality is due to Murakami and Murakami [30]. In [3; 4; 5] we quoted occasionally the first one as a motivating fact. Later we realized that we were unable to derive a complete proof from the existing literature (in particular Kashaev [23;24]), so we provided an independent one in [6], under the ambiguity $\equiv_{2 N}$. The above statement with $\equiv_{N}$ follows from the state sum sign correction introduced in the present paper (see also Remarks 2.11 and 5.5).

When $\partial V \neq \varnothing$ the QHFT partition functions are more sophisticated; see [5]. In particular the link $L$ contains an essential simple closed curve on each boundary component which actually encodes a Dehn filling instruction. Anyway, also in this case the invariants $\mathcal{H}_{N}(V, L, \rho,(h, k))$ are defined for arbitrary characters and weights. 
Relation with the QHI of cusped manifolds The patterns over cusped manifolds and the QHFT patterns are complementary in the sense that the link $L$ is empty in the former. In $[4 ; 5]$ the proof of invariance of $\mathcal{H}_{N}(M)=\mathcal{H}_{N}\left(V, \rho_{\text {hyp }},(0,0)\right)$ differs to many extents from the one for the QHFT partition functions. It uses the volume rigidity for cusped manifolds (see Francaviglia [19], for example), Thurston's hyperbolic Dehn filling theorem, a construction of certain auxiliary invariants $\mathcal{H}_{N}\left(V, \rho_{\text {hyp }},(0,0), a\right)$ that depend a priori on an additional datum $a$, and finally a surgery formula. Set $H_{N}(W, L, \rho):=\mathcal{H}_{N}(W, L, \rho, 0)$, where $W$ is closed (see our notations above). By combining all these results we proved the following.

Theorem 1.4 [5, Section 6.2] Let $W_{n}$ be a sequence of closed hyperbolic Dehn fillings of $M$ whose holonomies $\rho_{n}$ (considered as $\operatorname{PSL}(2, \mathbb{C})$-characters on $M$ ) converge to $\rho_{\text {hyp }}$ in $X(M)$. Denote by $L_{n}$ the geodesic core of the solid torus that fills $V$ to produce $W_{n}$. For every odd $N \geq 3$ and every additional datum $a$ we have

$$
\lim _{n \rightarrow \infty} H_{N}\left(W_{n}, L_{n}, \rho_{n}\right) \equiv_{2 N} \mathcal{H}_{N}\left(V, \rho_{\text {hyp }},(0,0), a\right) .
$$

Hence $a$ is eventually immaterial, and the limit defines $\mathcal{H}_{N}(M) \in \mathbb{C} / \mu_{2 N}$.

The normalization $(h, k)=(0,0)$ on the right side of (4) is a byproduct of the proof. Under some additional assumptions on $M$ (for instance if $M$ is very gentle according to $[4 ; 5]$; then $a=\varnothing)$, we can avoid the delicate surgery argument and define the invariants $\mathcal{H}_{N}\left(V, \rho_{\text {hyp }}, h, k\right)$ for arbitrary weights relative to the hyperbolic holonomy.

\section{C State sums over weakly branched triangulations}

One complication with the construction of the QHI of cusped manifolds in [4; 5] depends on a technical difficulty that we overcome in the present paper.

For every topological support $Y_{\mathcal{P}}$, denote by $\widehat{V}$ the compact space obtained by filling each boundary component of $\partial V$ with the cone over it. If $\partial V=\varnothing$ then $\widehat{V}=W$; $\widehat{V}$ has a finite set of nonmanifold points, the vertices of the filling cones. For every pattern $\mathcal{P}$ supported by $Y_{\mathcal{P}}, \mathcal{H}_{N}(\mathcal{P})$ is computed by state sums over certain decorated triangulations $T$ of $\hat{V}$, depending on the choice of a point $z_{\rho}$ in the associated gluing variety $G(T)$ such that $z_{\rho}$ represents the character $\rho$ (see Section 2), and on a suitable encoding of the weights $(h, k)$. Moreover, $T$ is equipped with a branching; equivalently, $T$ carries a structure of $\Delta$-complex in the sense of Hatcher [21].

In the case of QHFT partition functions this is not so demanding: $T$ can be a quasiregular triangulation (every edge has distinct endpoints), a branching $b$ can be induced, for example, by a total ordering of the vertices, and $z_{\rho}$ can be realized by means of a so-called idealization of PSL $(2, \mathbb{C})$-valued 1-cocycles on $(T, b)$. 
On the other hand, for a cusped manifold $M$ we use ideal triangulations $T$ of $\widehat{V}$ such that the gluing variety $G(T)$ contains a point $z_{h}$ representing the hyperbolic holonomy $\rho_{\text {hyp }}$, and having coordinates with nonnegative imaginary part (we say that $z_{h}$ is nonnegative). Such triangulations exist for every $M$, for instance any maximal subdivision of the canonical Epstein-Penner cell decomposition has this property. However we do not know if every cusped manifold has such a triangulation $T$ admitting a branching $b$. For instance, the canonical Epstein-Penner decomposition of the figureeight knot sister (m003 in SnapPea's census) is made of two regular hyperbolic ideal tetrahedra and does not carry any branching. The very gentle manifolds $M$ mentioned after Theorem 1.4 admit by definition a branched triangulation $(T, b)$ with a nonnegative point $z_{h}$ in $G(T)$.

In order to get Theorem 1.1(1) we relax branchings to weak branchings, which exist on every triangulation, and this leads us to include 2-face tensors in the state sum formulas. In this setup, as well as to cover arbitrary characters of $M$ in $\Omega(M)$, the proof of the state sum invariance requires additional arguments with respect to $[4 ; 5]$.

\section{$1 D$ Plan of the paper}

Let $V$ be as in Section $1 \mathrm{~B}$ and $\widehat{V}$ as in Section 1C.

In Section 2 we recall a few general facts about triangulations endowed with prebranchings, weak branchings or branchings, and the associated gluing varieties of $M$.

In Section 3 we construct the analytic configuration $\mathcal{A}_{N}(T, \tilde{b}, c)$ for every odd integer $N \geq 3$, weakly branched triangulation $(T, \tilde{b})$ of $\hat{V}$, and rough charge $c$ on $T$ (suitably specialized global charges will eventually encode the $c$-weights below). In particular $\mathcal{A}_{N}(T, \tilde{b}, c)$ contains an infinite Abelian covering of the gluing variety, $p_{\infty}: G(T, \tilde{b})_{\infty} \rightarrow G(T)$, and an analytic function $\mathcal{H}_{N}(T, \tilde{b}, c): G(T, \widetilde{b})_{\infty} \rightarrow \mathbb{C}$. In the case of QHFT patterns it is described qualitatively by the following proposition.

Proposition 1.5 For every topological support $\left(V, L,\left(h_{c}, k_{c}\right)\right)$ there is a weakly branched triangulation $(T, \widetilde{b})$ of $\widehat{V}$ and a global charge $c$ on $T$ such that:

(1) $c$ encodes the $c$-weight $\left(h_{c}, k_{c}\right)$.

(2) For any QHFT pattern $(V, L, \rho,(h, k))$ with topological support $\left(V, L,\left(h_{c}, k_{c}\right)\right)$, there is a point $u \in G(T, \tilde{b})_{\infty}$ such that $p_{\infty}(u)$ represents the character $\rho$ and for every odd $N \geq 3$ the scalar $\tilde{\mathcal{H}}_{N}(V, L, \rho,(h, k)) \equiv_{2 N} \mathcal{H}_{N}(T, \tilde{b}, c)(u)$ does not depend on the choice of $(T, \tilde{b}, c)$ and $u$.

In the case of patterns over a cusped manifold $M$, the following holds. 
Proposition 1.6 For every topological support $Y=\left(V,\left(h_{c}, k_{c}\right)\right)$ over $M$, there is a determined Zariski open subset $\Omega(M)$ of $X_{0}(M)$ containing the hyperbolic holonomy $\rho_{\text {hyp }}$, and there is a weakly branched ideal triangulation $(T, \widetilde{b})$ of $\widehat{V}$, and a global charge $c$ on $T$ such that:

(1) $c$ encodes the $c$-weight $\left(h_{c}, k_{c}\right)$.

(2) The gluing variety $G(T)$ contains a nonnegative point $z_{h}$ representing $\rho_{\text {hyp }}$.

(3) There is an irreducible component $Z$ of $G(T)$, a Zariski open subset $\Omega_{Z}$ of $Z$ containing $z_{h}$, and a homeomorphism $\rho: \Omega_{Z} \rightarrow \Omega(M)$ extending a regular rational isomorphism between Zariski open subsets, such that $\rho(z)$ is the holonomy of $V$ represented by $z$.

(4) For every pattern $(V, \rho,(h, k))$ over $M$ with topological support $Y$ and for every $\rho \in \Omega(M)$, there is a point $u \in Z_{\infty}:=p_{\infty}^{-1}(Z)$ such that $\rho=\rho\left(p_{\infty}(u)\right)$, and for every odd $N \geq 3$ the scalar $\mathcal{H}_{N}(V, \rho,(h, k)) \equiv_{2 N} \mathcal{H}_{N}(T, \tilde{b}, c)(u)$ does not depend on the choice of $(T, \tilde{b}, c)$ and $u$.

We will use concrete models of the finite coverings and regular rational maps in Theorem 1.1(2) by considering a suitable factorization of $\mathcal{H}_{N}(T, \widetilde{b}, c): Z_{\infty} \rightarrow \mathbb{C}$.

In Section 4 we develop the content of Proposition 1.6 and prove Theorem 1.1(1)-(2) and the analogous result when $N=1$ (see comment (c) and Corollary 4.17). In Section 5 we indicate briefly how to deal with QHFT partition functions. In Section 6 we collect a few facts about a diagrammatic calculus for weakly branched triangulations. This calculus is used in Section 7, which contains the invariance proof of the state sums defined over such triangulations.

In Section 8 we prove Theorem 1.1(3) and the analogous result for QHFT partition functions. As a byproduct we show that global compensations of the local sign ambiguities imply that the QHI $\mathcal{H}_{N}(\mathcal{P})$ defined by means of branched triangulations have no sign ambiguity. This applies to QHFT partition functions and to very gentle cusped manifolds.

In Section 9 we describe the quantum hyperbolic invariants of three cusped manifolds: the figure-eight knot complement, its sister, and the complement of the knot $5_{2}$. We present the results of numerical computations showing that the functions $\mathcal{H}_{N}^{h_{f}, h_{c}, k_{c}}$ depend on the weights as $N$ approaches $+\infty$, and recover the volume for some specific choices of the weights.

Notation In general we denote by $N$ the odd integers $\geq 3$, including the case $N=1$ when specified, and we put $m:=(N-1) / 2$ and $\zeta:=\exp (2 \pi i / N)$. The set $\mathcal{I}_{N}=$ $\{0, \ldots, N-1\}$ is identified with the group $\mathbb{Z} / N \mathbb{Z}$ and $[n]_{N} \in \mathcal{I}_{N}$ denotes the remainder modulo $N$ of $n \in \mathbb{N}$. We let $\delta_{N}(n):=1$ (resp. 0$)$ if $[n]_{N}=0\left(\right.$ resp. $\left.[n]_{N} \neq 0\right)$. 
Acknowledgments The first author's work was supported by the ANR projects Géométrie et Topologie Quantiques (ANR-08-JCJC-0114-01) and Extensions des théories de Teichmüller-Thurston (ANR-09-BLAN-0116-01). Many thanks are due to Columbia University, Fukuoka University, the MSC at Tsinghua University and the University of Utah at Moab, where parts of this work was presented. The second author's work is supported by the Italian FIRB project Geometry and topology of low-dimensional manifolds. The numerical computations presented in Section 9 were obtained using the Maple software.

\section{Structured triangulations and gluing varieties}

Triangulations Let $V$ be as in Section 1B, that is, a compact oriented connected 3-manifold with (possibly empty) boundary $\partial V$ made by toric components. Denote by $\hat{V}$ the space obtained by taking the cone over each boundary component of $\partial V$. A triangulation $T$ of $\widehat{V}$ is a collection of oriented tetrahedra $\Delta_{1}, \ldots, \Delta_{s}$ together with a complete system $\sim$ of pairings of their 2 -faces via orientation reversing affine isomorphisms, such that the oriented quotient space

$$
T:=\coprod_{i=1}^{s} \Delta_{i} / \sim
$$

is homeomorphic to $\hat{V}$, preserving the orientations. We will distinguish between the 2-faces, edges and vertices of the disjoint union $\bigsqcup_{i=1}^{S} \Delta_{i}$, and the ones of $T$ after the 2-face pairings. In particular we denote by $E\left(\left\{\Delta_{i}\right\}\right)$ and $E(T)$ the set of edges of $\coprod_{i=1}^{S} \Delta_{i}$ and $T$ respectively, and we write $E \rightarrow e$ to mean that an edge $E \in E\left(\left\{\Delta_{i}\right\}\right)$ is identified to $e \in E(T)$ under the 2 -face pairings. The 2 -faces of each tetrahedron $\Delta_{i}$ have the boundary orientation defined by the rule: first the outgoing normal. When $\partial V \neq \varnothing$, the nonmanifold points of $\hat{V}$ are necessarily vertices of $T$. A triangulation $T$ of $\hat{V}$ is called ideal if the set of vertices of $T$ coincides with the set of nonmanifold points of $\widehat{V}$.

Gluing varieties Let $T$ be as above. For every tetrahedron $\Delta_{j}$ choose a vertex $v^{j}$. Order the edges of the opposite 2 -face $F^{j}$ so that the induced cyclic ordering is the opposite of the boundary orientation. Denote these edges by $E_{0}^{j}, E_{1}^{j}, E_{2}^{j}$. Give a label $u_{r}^{j} \in \mathbb{C}_{*}$ to $E_{r}^{j}$ and the opposite edge, where $\mathbb{C}_{*}:=\mathbb{C} \backslash\{0,1\}$ and $r \in \mathbb{Z} / 3 \mathbb{Z}$. The gluing variety of $T$ is the algebraic subset of $\mathbb{C}_{*}^{3 s}$ with coordinates $\left(u_{0}^{1}, u_{1}^{1}, u_{2}^{1}, \ldots, u_{0}^{s}, u_{1}^{s}, u_{2}^{s}\right)$ and defining equations, for all $j \in\{1, \ldots, s\}, r \in \mathbb{Z} / 3 \mathbb{Z}$ and $e \in E(T)$,

$$
u_{r+1}^{j}\left(1-u_{r}^{j}\right)=1, \quad \prod_{E \rightarrow e} u(E)=1 .
$$


By the first set of $s$ tetrahedral relations, we see that the gluing variety is the graph of an explicit regular rational map defined on an algebraic subset of $\mathbb{C}_{*}^{S}$ defined by the second set of edge relations. The auxiliary choices of ordered edges $E_{r}^{j}$ being immaterial, these algebraic varieties are canonically isomorphic. We denote them by $G(T)$.

Every point $u \in G(T)$ represents a $\operatorname{PSL}(2, \mathbb{C})$-character $\rho(u)$ of $V$. Its components $u_{0}^{j}, u_{1}^{j}, u_{2}^{j}$ can be interpreted as the cross ratio parameters of an isometry class of oriented hyperbolic ideal tetrahedra associated to $\Delta_{j}$, that we denote by $\left(\Delta_{j}, u^{j}\right)$. Their imaginary parts have a same sign $\epsilon^{j} \in\{-1,0,1\}$ (by convention $\epsilon^{j}=0$ if the imaginary parts are zero). By a classical result of Schläffli, the algebraic volume of $\left(\Delta_{j}, u^{j}\right)$ is given by

$$
\operatorname{Vol}_{\text {alg }}\left(\Delta_{j}, u^{j}\right):=\epsilon\left(u^{j}\right) \operatorname{Vol}\left(\Delta_{j}, u^{j}\right)=\mathrm{D}_{2}\left(u_{0}^{j}\right),
$$

where Vol is the geometric positive volume and $\mathrm{D}_{2}$ the Bloch-Wigner dilogarithm. When the components $u_{r}^{j}$ are real, $\left(\Delta_{j}, u^{j}\right)$ is degenerate and both sides of (5) vanish. By summing the algebraic volumes of the $\left(\Delta_{j}, u^{j}\right) \mathrm{s}$ we get a volume function

$$
\text { Vol: } G(T) \rightarrow \mathbb{R} \text {. }
$$

If $G(T)$ is nonempty, for every point $u \in G(T), \operatorname{Vol}(u)$ coincides with the (intrinsically defined) volume $\operatorname{Vol}(\rho(u))$ of the character $\rho(u)$. In general $G(T)$ might be empty, but $\hat{V}$ always has triangulations $T$ such that $G(T)$ is nontrivial. A first general result concerns its dimension.

Theorem $2.1 \quad[31 ; 33 ; 7]$ Assume that $V$ has one torus boundary component. Let $T$ be an ideal triangulation of $\hat{V}$. If the gluing variety $G(T)$ is nonempty, then it is a complex algebraic set of dimension $\geq 1$.

This result depends on the combinatorial properties of $T$. If the interior of $V$ is a cusped manifold $M$, then it has the canonical Epstein-Penner (EP) cell decomposition by embedded convex hyperbolic ideal polyhedra; see for instance [7].

Proposition 2.2 The maximal subdivisions of the EP cell decomposition of $M$ define a finite set $\mathcal{T}_{\mathrm{EP}}(M)$ of ideal triangulations of $\widehat{V}$, such that for every $T \in \mathcal{T}_{\mathrm{EP}}(M)$ the gluing variety $G(T)$ contains a nonnegative point $u_{h} \in G(T)$ such that $\rho\left(u_{h}\right)=\rho_{\text {hyp }}$ and $\operatorname{Vol}\left(u_{h}\right)=\operatorname{Vol}(M)$.

Every nondegenerate hyperbolic ideal tetrahedron of $\left(T, u_{h}\right)$ has strictly positive (geometric) volume, but in general one cannot avoid some degenerate tetrahedra.

For other manifolds the nature of $G(T)$ is not so well known. For instance consider the case of a closed 3-manifold $W$. A triangulation $T$ of $W$ is called quasiregular if 
every edge of $T$ has distinct vertices in $T$. It is clear that every triangulation has a quasiregular subdivision. Take one, and fix a total ordering of the vertices. For every edge $e$ with endpoints $v$ and $v^{\prime}$, orient $e$ from $v$ to $v^{\prime}$ if $v<v^{\prime}$. Every simplicial $\operatorname{PSL}(2, \mathbb{C})$-valued 1 -cocycle $z$ on $T$, defined by using this edge orientation, represents a character $\rho(z)$ of $W$. Then to any sufficiently generic cocycle $z$ one can associate a point $u \in G(T)$ such that $\rho(z)=\rho(u)$, which leads to the following.

Proposition 2.3 [3] Let $T$ be a quasiregular triangulation of $W$. For every character $\rho$ of $W$ there is a point $u \in G(T)$ such that $\rho=\rho(u)$.

Clearly, the point $u$ is far from unique. For instance, if $W=S^{3}$ every point of $G(T)$ represents the trivial character. A similar, slightly more elaborated result holds for all other topological supports of QHFT patterns; it uses triangulations of $\widehat{V}$ obtained from quasiregular relative triangulations $(T, \partial T)$ of $(V, \partial V)$ by adding a cone over each component of $\partial T$.

The method used to prove Proposition 2.3 is reminiscent of Thurston's spinning construction, and is strictly related to it when $W$ is hyperbolic. In that case, the following result, which is proved by using the spinning construction, agrees with Proposition 2.3 in the case of quasiregular triangulations.

Proposition 2.4 [28] Let $T$ be a triangulation of a closed oriented hyperbolic 3 manifold $W$ such that no edge is a null-homotopic loop in $W$. Then there exists $u \in$ $G(T)$ such that $\operatorname{Vol}(u)=\operatorname{Vol}(W)$, and moreover its holonomy $\rho(u)$ is the hyperbolic holonomy.

Variations on branched triangulations Define a prebranched tetrahedron $(\Delta, \sigma)$ as an oriented tetrahedron $\Delta$ with a choice $\sigma$ of coorientations of the 2-faces, such that two coorientations are ingoing and two are outgoing. As every 2 -face has the boundary orientation, by duality $\sigma$ can be interpreted as a system of 2 -face orientations.

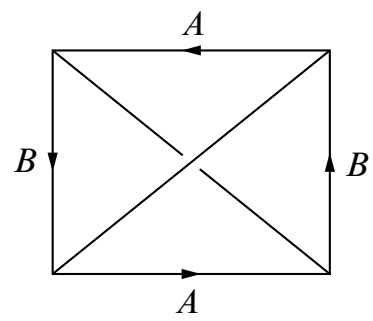

Figure 1: Prebranched tetrahedron

Figure 1 shows a prebranched tetrahedron $(\Delta, \sigma)$ embedded in $\mathbb{R}^{3}$, with coordinates $\left(x_{1}, x_{2}, x_{3}\right)$ such that the plane of the picture is $\left\{x_{3}=0\right\}$. We put on $\Delta$ the orientation 
induced from $\mathbb{R}^{3}$, and assume that the two 2 -faces above (resp. below) the plane are those with outgoing (resp. ingoing) coorientations. This specifies two diagonal edges and four square edges. Every square edge is oriented as the common boundary edge of two 2-faces with opposite coorientations. So the square edges form an oriented quadrilateral. Using the orientation of $\Delta$, one can also distinguish among the square edges two pairs of opposite edges, called $A$-edges and $B$-edges. The orientation of the diagonal edges is not determined.

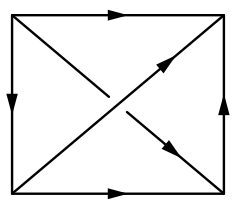

$A$

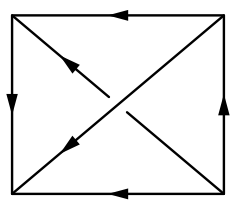

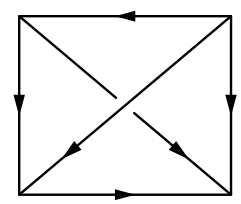

$B$

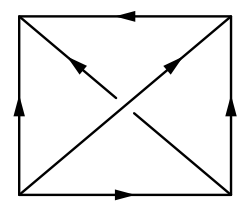

Figure 2: Branched tetrahedra inducing the same prebranched tetrahedron

An oriented tetrahedron $\Delta$ becomes a 3 -simplex by ordering its vertices. This is equivalent to a system $b$ of orientations of the edges, called (local) branching, such that the vertex $v_{j}$ has $j$ incoming edges, $j \in\{0, \ldots, 3\}$. The 2 -faces of $(\Delta, b)$ are ordered as the opposite vertices, and $b$ induces a branching $b_{F}$ on each 2-face $F$. The branchings $b$ and $b_{F}$ define orientations on $\Delta$ and $F$, respectively: the $b$-and $b_{F}$-orientations. The $b$-orientation may coincide or not with the orientation of $\Delta$. We encode this by a sign, $*_{b} \in\{-1,+1\}$. The boundary orientation and the $b_{F}$-orientation agree on two 2-faces. Hence $b$ defines a prebranching $\sigma_{b}$. On the other hand, given a prebranching $\sigma$ on $\Delta$ there are exactly four branchings such that $\sigma_{b}=\sigma$. They can be obtained by choosing an $A$ (resp. $B$ ) edge, reversing its orientation, and completing the resulting square edge orientations to define a branching $b$ (this can be done in a single way; see Figure 2). Note that $*_{b}=1$ (resp. $*_{b}=-1$ ) if and only if we have chosen an $A$ (resp. $B$ ) square edge, and the square edge is $\left[v_{0}, v_{3}\right]$. The diagonal edges are $\left[v_{0}, v_{2}\right]$ and $\left[v_{1}, v_{3}\right]$.

One can extend these notions to triangulations $T$ of $\hat{V}$ :

- A prebranched triangulation $(T, \sigma)$ is formed by prebranched tetrahedra $\left(\Delta_{j}, \sigma_{j}\right)$ such that the 2-face coorientations match under the 2 -face pairings. 
- A weakly branched triangulation $(T, \tilde{b})$ is formed by branched tetrahedra $\left(\Delta_{j}, b_{j}\right)$ such that the induced prebranched tetrahedra $\left(\Delta_{j}, \sigma_{b_{j}}\right)$ form a prebranched triangulation $(T, \sigma)$.

- A branched triangulation $(T, b)$ is formed by branched tetrahedra $\left(\Delta_{j}, b_{j}\right)$ such that the branchings (ie the edge orientations) match under the 2 -face pairings.

Remark 2.5 Branched triangulations of $\widehat{V}$ and $\Delta$-complexes over $\widehat{V}$ [21] are equivalent notions. In particular the simplicial 3 -chain $\sum_{j} *_{b_{j}}\left(\Delta_{j}, b_{j}\right)$ represents the fundamental class in $H_{3}(\widehat{V} ; \mathbb{Z})$.

Let $T$ be a triangulation of $\hat{V}$. Denote by $\bar{V}$ the compact 3-manifold with boundary obtained by removing a small open 3-ball around every vertex of $T$ which is a manifold point. Clearly, $V=\bar{V}$ if and only if $T$ is an ideal triangulation. A prebranched triangulation $(T, \sigma)$ of $\widehat{V}$ can be described in a very concrete way in terms of the standard spine $P$ of $\bar{V}$ dual to $T$.

Lemma 2.6 There is a one-to-one duality correspondence between the sets of prebranchings of $T$ and those of $P$, where a prebranching of $P$ is defined as an orientation of the singular locus $\operatorname{Sing}(P)$ such that every vertex has two outgoing and two ingoing edges.

The proof is evident, as every edge of $\operatorname{Sing}(P)$ is dual to a 2 -face of $T$. The notion of (weakly) branched triangulation $(T, \tilde{b})$ has a natural dual counterpart as (weakly) branched spine $(P, \bar{b})$.

The branched boundary of a prebranched triangulation The triangulation $T$ of $\widehat{V}$ yields a decomposition of $\bar{V}$ into truncated tetrahedra. Their triangular 2-faces form a triangulation $\partial T$ of $\partial \bar{V}$. If $(T, \sigma)$ is a prebranched triangulation, then $\partial T$ has a branching $b_{\partial \sigma}$ defined on the 2 -faces of $\partial T$ as in Figure 3.

The corners of every 2 -simplex of $\left(\partial T, b_{\partial \sigma}\right)$ have a label in $\{A, B, \varnothing\}$, and a sign \pm 1 obtained by comparing the boundary and the $b_{\partial \sigma}$-orientations. Such a $\{A, B, \varnothing\}-$ labeling may be defined more generally for any branched triangulation of a closed oriented surface.

Lemma 2.7 Each vertex of a branched triangulation of a closed oriented surface has an even number of adjacent corners with the empty label $\varnothing$. Hence every edge $e$ of a prebranched triangulation $(T, \sigma)$ of $\hat{V}$ has an even number of diagonal edges $E \in E\left(\left\{\Delta_{i}\right\}\right)$ such that $E \rightarrow e$. 

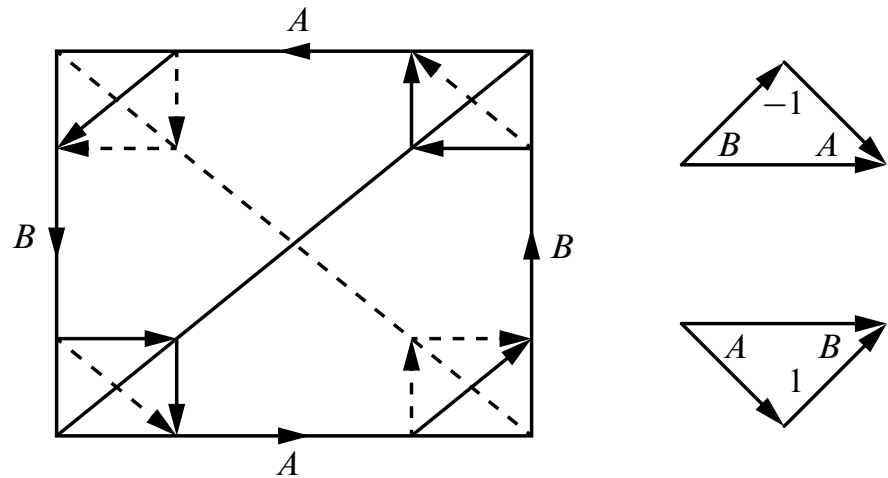

Figure 3: Branched boundary of prebranched triangulations

Proof For each 2-face the edges adjacent to the corner with label $\varnothing$ have opposite $b$-orientations, while they agree elsewhere. The existence of an orientation on any transverse loop implies the first claim. The second follows by considering the triangulation $\partial T$.

Remark 2.8 Give the labels $1 \in \mathbb{Z} / 2 \mathbb{Z}$ to the diagonal edges and $0 \in \mathbb{Z} / 2 \mathbb{Z}$ to the square edges of every prebranched tetrahedron $(\Delta, \sigma)$ of $(T, \sigma)$. Then Lemma 2.7 and Lemma 2.10 below imply that $\mathbb{Z}_{2}$-valued taut structures on $T$ always exist (a notion borrowed from F Luo's work).

Networks and $\mathcal{N}$-graphs Consider an oriented graph $(\operatorname{Sing}(P), \bar{\sigma})$ as in Lemma 2.6. Put around every vertex $v$ the dual branched tetrahedron $\left(\Delta_{v}, b_{v}\right)$ so that its 2 -faces intersect transversely the edges of $\operatorname{Sing}(P)$. Thus each edge connects a 2-face $F^{i}$ of the initial branched tetrahedron $\left(\Delta_{v_{i}}, b_{v_{i}}\right)$ with a 2 -face $F^{f}$ of the final one $\left(\Delta_{v_{f}}, b_{v_{f}}\right)$, identified in $T$. The gluing map $\phi_{e}: F^{i} \rightarrow F^{f}$ is determined by a color $r(e) \in \mathbb{Z} / 3 \mathbb{Z}$ defined as follows. Set $J_{3}=\{0,1,2\}$. Denote by $S\left(J_{3}\right)$ the symmetric group on $J_{3}$, by $A\left(J_{3}\right)$ the subgroup of even permutations, and by $u_{j}^{i}$ and $u_{j}^{f}$ the vertices of $F^{i}$ and $F^{f}$, with $j \in J_{3}$. The map $\phi_{e}$ is determined by the permutation $\tau_{e} \in A\left(J_{3}\right)$ such that $\phi_{e}\left(u_{j}^{i}\right)=u_{\tau_{e}(j)}^{f}$. Then we set

$$
r(e):=\alpha^{-1}\left(\tau_{e}\right) \in \mathbb{Z} / 3 \mathbb{Z},
$$

where $\alpha: \mathbb{Z} / 3 \mathbb{Z} \rightarrow A\left(J_{3}\right)$ is the isomorphism given by $\alpha(j)=(012)^{j}$. We define $\mathcal{N}(T, \tilde{b})$ as the oriented graph $(\operatorname{Sing}(P), \bar{\sigma})$ endowed with the $\mathbb{Z} / 3 \mathbb{Z}$-edge colors $r(e)$, and with the correspondence, for every vertex $v$, between the 2 -faces of $\left(\Delta_{v}, b_{v}\right)$ and the germs of edges adjacent to $v$. Clearly we have the following.

Lemma 2.9 A weakly branched triangulation $(T, \tilde{b})$ is branched if and only if all the $\mathbb{Z} / 3 \mathbb{Z}$-edge colors $r(e)$ are equal to 0 . 
Lemma 2.10 Any triangulation $T$ of $\widehat{V}$ admits prebranchings and hence compatible weak-branchings.

We stress that this is no longer true for genuine branchings; see an example in Figure 7. If $T$ is quasiregular, then every total ordering of the vertices induces a branching of $T$.

It is useful to represent $\mathcal{N}(T, \tilde{b})$ by an $\mathcal{N}$-graph $\Gamma$, defined as follows. Basically $\Gamma$ is a planar immersion of $(\operatorname{Sing}(P), \bar{\sigma})$ with normal crossings. It has two kinds of vertices: essential crossings, represented by a solid dot, corresponding to the vertices of $P$ and connected by the oriented edges of $\Gamma$, and nonessential ("immaterial") crossings due to the immersion. An essential crossing encodes a branched tetrahedron $\left(\Delta_{v}, b_{v}\right)$ as shown in Figure 4; the labels on the arc endpoints correspond to the $b_{v}$-ordering of the dual 2-faces of $\left(\Delta_{v}, b_{v}\right)$. A full decoding is provided by Figure 5 in the case $*_{b}=1$, showing a branched tetrahedron, the dual "butterfly" with cooriented (hence oriented) wings, and its conversion into a portion of an oriented branched surface. An arc of $\Gamma$ corresponding to an edge $e$ of $(\operatorname{Sing}(P), \bar{\sigma})$ inherits the $\mathbb{Z} / 3 \mathbb{Z}$-color $r(e)$. If $r(e)=0$ we omit it.
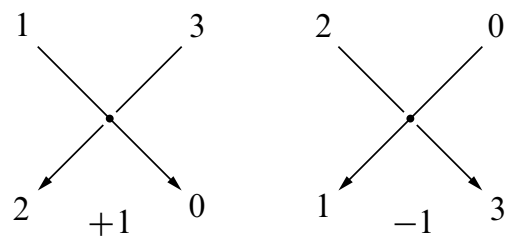

Figure 4: $\mathcal{N}$-graph crossings for $*_{b}= \pm 1$
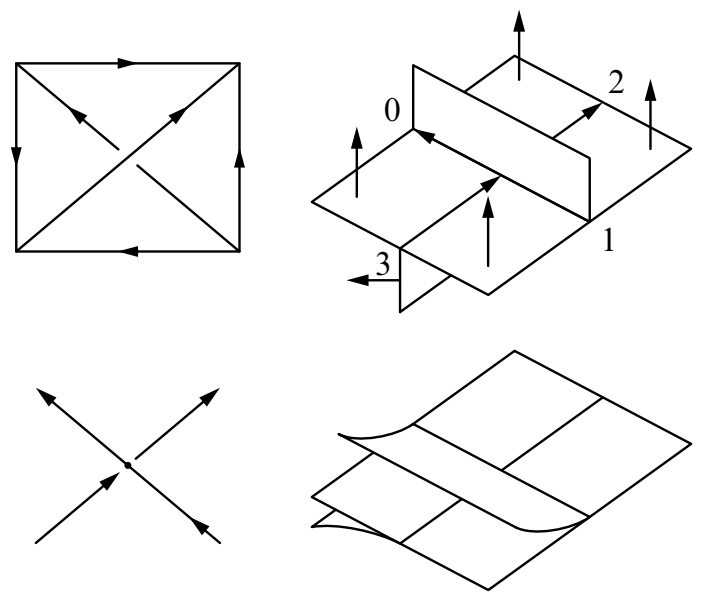

Figure 5: Decoding of an $\mathcal{N}$-graph crossing $\left(*_{b}=1\right)$ 
Given $\Gamma$, an easy decoding procedure (extending the one of Figure 5) produces an embedding in $\mathbb{R}^{3}$ of a closed regular neighborhood $N(P)$ of $\operatorname{Sing}(P)$ in the standard spine $P$. We believe that it is enough to show this in the case of the simplest cusped manifolds: $M_{0}$, the figure-eight-knot complement in $S^{3}$, and its sister $M_{1}$, which is the complement of a knot in the lens space $L_{5,1}$; see [29]. In both cases the EpsteinPenner decomposition is an ideal triangulation made by two regular hyperbolic ideal tetrahedra. Denote by $T_{0}$ and $T_{1}$ the corresponding triangulations of $\widehat{V}_{0}$ and $\widehat{V}_{1}$. Figure 6 (resp. Figure 7) shows an $\mathcal{N}$-graph and its decoding for a branching $b$ of $T_{0}$ (resp. weak branching $\widetilde{b}$ of $T_{1}$ ). It is not hard to verify that $T_{1}$ does not carry any branching.
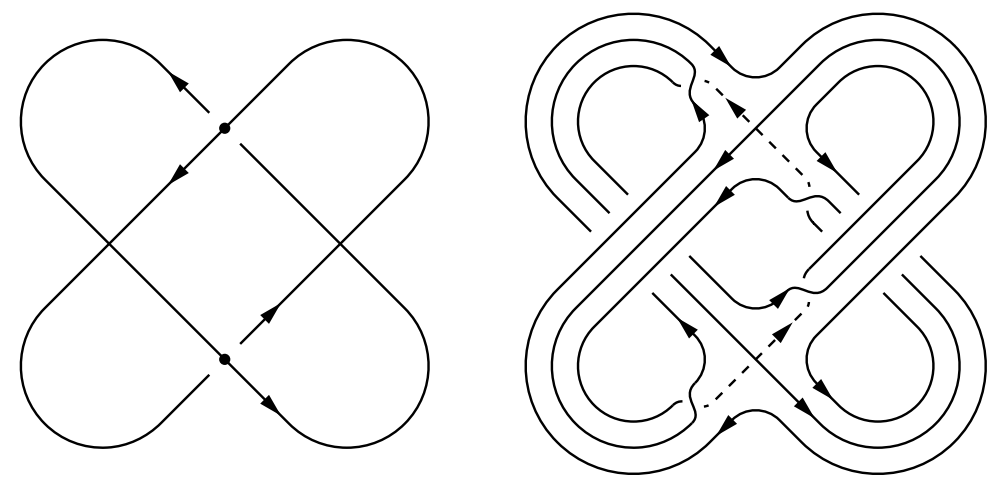

Figure 6: A $\mathcal{N}$-graph of $\left(T_{0}, b\right)$ and its decoding
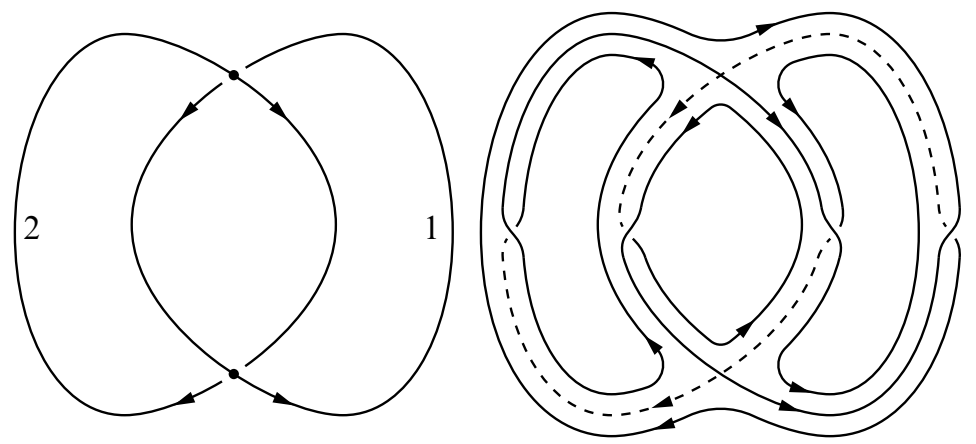

Figure 7: A $\mathcal{N}$-graph of $\left(T_{1}, \widetilde{b}\right)$ and its decoding

Edge decorations We will use several labelings of the edges $E \in E\left(\left\{\Delta_{i}\right\}\right)$ of a weakly branched triangulation, called decorations, such that for every tetrahedron, opposite edges have the same label. An example is the decoration of the square 

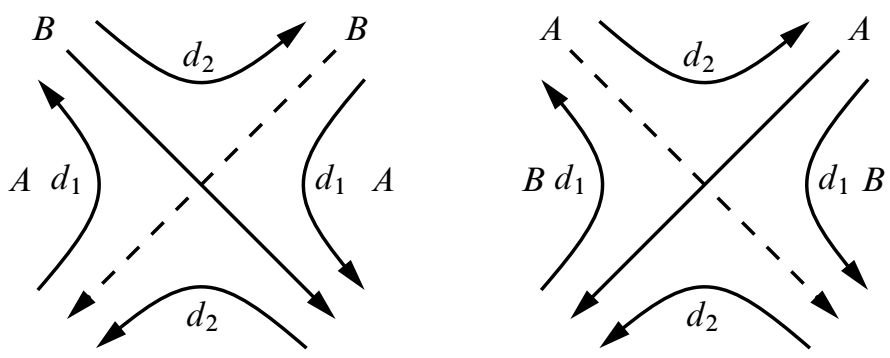

Figure 8: Edge decorations on $\mathcal{N}$-graphs

edges with $A$ or $B$ according to the prebranching, and of the diagonal edges with $\varnothing$. Every decoration $d$ is determined on each tetrahedron $\Delta_{j}$ by the triple $\left(d_{0}, d_{1}, d_{2}\right):=$ $\left(d\left(E_{0}^{j}\right), d\left(E_{1}^{j}\right), d\left(E_{2}^{j}\right)\right)$. In terms of decoded $\mathcal{N}$-graphs, they are placed as in Figure 8, where we understand that the over/under crossing arcs are labeled by $d_{0}$, and we show also $A, B, \varnothing$.

Remark 2.11 In the case of genuine branchings, $\mathcal{N}$-graphs were used in [6] under the name of normal o-graphs, with the opposite convention for the sign $*_{b}$ (in accordance with the usual crossing signs of link diagrams). This choice gives the equality $H_{N}\left(S^{3}, L\right) \equiv{ }_{N} J_{N}(\bar{L})$, where $\bar{L}$ is the mirror image of the link $L$. The present convention yields the statement of Theorem 1.2.

The model $\boldsymbol{G}(\boldsymbol{T}, \tilde{\boldsymbol{b}})$ of the gluing variety If $(T, \tilde{b})$ is a weakly branched triangulation, we can use the weak branching $\tilde{b}$ to fix the auxiliary choices used in the definition of the gluing variety $G(T)$. On every branched tetrahedron $\left(\Delta_{j}, b_{j}\right)$ let $v^{j}=v_{3}^{j}$, and order the edges of $F_{3}^{j}$ as $E_{0}^{j}=\left[v_{0}^{j}, v_{1}^{j}\right], E_{1}^{j}=\left[v_{1}^{j}, v_{2}^{j}\right]$, and $E_{2}^{j}=\left[v_{0}^{j}, v_{2}^{j}\right]$. If $*_{b_{j}}=+1$, this ordering is compatible with the cyclic ordering induced by the opposite of the boundary orientation of $F_{3}^{j}$. If $*_{b_{j}}=-1$ this is no longer true, and since the coordinates $u_{r}^{j}, r \in \mathbb{Z} / 3 \mathbb{Z}$, of $G(T)$ are the cross ratio moduli of an isometry class of oriented hyperbolic tetrahedron associated to $\Delta_{j}, u_{r}^{j}$ should be replaced by $\left(u_{r}^{j}\right)^{-1}$ in order to compensate the choice of opposite orientation. Hence we define a new system of coordinates $\left(w_{r}^{j}\right) \in \mathbb{C}_{*}^{3 s}$ of $G(T)$ by labeling $E_{r}^{j}$ with $w_{r}^{j}$, setting

$$
\left(w_{0}^{j}, w_{1}^{j}, w_{2}^{j}\right)= \begin{cases}\left(u_{0}^{j}, u_{1}^{j}, u_{2}^{j}\right) & \text { if } *_{b_{j}}=+1 \\ \left(1 / u_{2}^{j}, 1 / u_{1}^{j}, 1 / u_{0}^{j}\right) & \text { if } *_{b_{j}}=-1\end{cases}
$$

This gives the model of $G(T)$ we are going to use. We denote it by $G(T, \tilde{b})$. The defining equations of $G(T, \widetilde{b})$ are the edge relations, for all edges $e$ of $T$, given by

$$
\prod_{E \rightarrow e} w(E)^{* E}=1
$$


where if $E$ is an edge of $\left(\Delta_{j}, b_{j}\right)$ we have $w(E)=w_{i}^{j}$ if and only if $E$ is $E_{i}^{j}$ or the opposite edge, and $*_{E}:=*_{b_{j}}$. The volume function on $G(T, \widetilde{b})$ takes now the form $\operatorname{Vol}(w)=\sum_{j} *_{b_{j}} \mathrm{D}_{2}\left(w_{0}^{j}\right)$. Clearly $\operatorname{Vol}(u)=\operatorname{Vol}(w(u))$.

Example 2.12 It is easy to recover the edge equations of $G(T, \tilde{b})$ from a decoded $\mathcal{N}$-graph representing it: at each essential crossing one places the cross ratio variables $\left(w_{0}, w_{1}, w_{2}\right)$ like the decorations $\left(d_{0}, d_{1}, d_{2}\right)$ in Figure 8, and take the products of cross ratio variables along the boundary lines of $N(P)$. For example, consider the cusped manifold $M_{1}$ and $\left(T_{1}, \tilde{b}\right)$. Assign $\left(w_{0}, w_{1}, w_{2}\right)$ to the top crossing of Figure 7 , and $\left(W_{0}, W_{1}, W_{2}\right)$ to the bottom one. Note that $*_{b}=+1$ for both of them. Then we get the equations

$$
w_{0} w_{1}^{2} W_{0} W_{1}^{2}=1, \quad w_{0} w_{2}^{2} W_{0} W_{2}^{2}=1 .
$$

Using the relation $w_{j+1}=1 /\left(1-w_{j}\right)$ and the similar one for $W_{j},(9)$ reduces to the unique quadratic equation

$$
w_{1}\left(w_{1}-1\right) W_{1}\left(W_{1}-1\right)=1 .
$$

The parameter space of positive solutions is the half plane $\operatorname{Im} w_{1}>0$ with the ray $0.5+s i$ removed, where $\sqrt{15} / 2 \leq s<+\infty$ [29]. The complete hyperbolic structure is realized at $w_{1}=W_{1}=\exp (i \pi / 3)$ (two regular ideal tetrahedra). Similarly, using $\left(T_{0}, b\right)$ one recovers Thurston's celebrated treatment of the figure-eight knot complement $M_{0}$.

\section{Analytic configurations}

\section{A Local analytic configurations}

Take an oriented 3 -simplex $(\Delta, b)$. As in the previous section the $2-$ face $F_{3}$ is opposite to the vertex $v_{3}$, and the edges of $F_{3}$ are ordered as

$$
E_{0}=\left[v_{0}, v_{1}\right], \quad E_{1}=\left[v_{1}, v_{2}\right], \quad E_{2}=\left[v_{0}, v_{2}\right] .
$$

3A1 Quantum hyperbolic 3-simplices Recall that the edge decorations of $(\Delta, b)$ are equal on opposite edges, and hence specified by triples $d=\left(d_{0}, d_{1}, d_{2}\right)$, where $d_{r}=d\left(E_{r}\right)(r \in \mathbb{Z} / 3 \mathbb{Z})$. A quantum hyperbolic 3 -simplex is a tuple $(\Delta, b, w, f, c)$, where:

- $w=\left(w_{0}, w_{1}, w_{2}\right)$ is the system of cross ratios (7).

- $f=\left(f_{0}, f_{1}, f_{2}\right) \in \mathbb{Z}^{3}$ is such that $l_{k}=\log \left(w_{k}\right)+i f_{k} \pi, k \in \mathbb{Z} / 3 \mathbb{Z}$, satisfy $l_{0}+l_{1}+l_{2}=0$.

- $c=\left(c_{0}, c_{1}, c_{2}\right) \in \mathbb{Z}^{3}$ satisfies $c_{0}+c_{1}+c_{2}=1$. 
Here, $\log$ is the branch of logarithm with imaginary part in $(-\pi, \pi]$. For every $k \in \mathbb{Z} / 3 \mathbb{Z}$ set

$$
l_{k, N, *_{b}, c}=\frac{1}{N}\left(\log \left(w_{k}\right)+i \pi(N+1)\left(f_{k}-*_{b} c_{k}\right)\right) .
$$

We call $f$ a flattening, $c$ a charge, $l_{k}$ a classical $\log$ branch, and $l_{k, N, *_{b}, c}$ a quantum $\log$ branch. If $\operatorname{Im}(w)>0$ (resp. $\operatorname{Im}(w)<0)$, then $c$ is a charge if and only if $-c$ (resp. $c$ ) is a flattening.

We organize the edge decorations $w, f, c$ as follows. Denote by $p_{\infty}: \mathcal{W}_{\infty} \rightarrow \mathbb{C}_{*}$ the maximal abelian covering map. We realize $\mathcal{W}_{\infty}$ as the quotient space $\mathcal{W}_{\infty}=$ $\left(\mathcal{D} \times \mathbb{Z}^{2}\right) / \sim$, where $\mathcal{D}$ is the result of gluing two copies of $(-\infty, 0)$ and $(1,+\infty)$ to the boundary of $\mathbb{C} \backslash((-\infty, 0) \cup(1,+\infty))$, and $\sim$ is the equivalence relation

$$
\begin{array}{ll}
(x+i 0 ; p, q) \sim(x-i 0 ; p+2, q) & \text { if } x \in(-\infty, 0), \\
(x+i 0 ; p, q) \sim(x-i 0 ; p, q+2) & \text { if } x \in(1,+\infty) .
\end{array}
$$

Setting $l(y ; m):=\log (y)+i m \pi, m \in \mathbb{Z}$, the bijective map

$$
\begin{aligned}
\left(x ; \frac{l(x ; p)-\log (x)}{i \pi}, \frac{l\left((1-x)^{-1} ; q\right)-\log \left((1-x)^{-1}\right)}{i \pi}\right) & \mapsto\left(l(x ; p), l\left((1-x)^{-1} ; q\right)\right)
\end{aligned}
$$

identifies $\mathcal{W}_{\infty}$ with the Riemann surface of the maps

$$
\phi_{\epsilon, \epsilon^{\prime}}: x \mapsto\left(\log (x)+i \epsilon \pi, \log \left((1-x)^{-1}\right)+i \epsilon^{\prime} \pi\right), \quad \epsilon, \epsilon^{\prime} \in\{0,1\} .
$$

For every $\operatorname{sign} *= \pm 1$ and couple $c=\left(c_{0}, c_{1}\right) \in \mathbb{Z}^{2}$ define the analytic map $l_{N, *, c}: \mathcal{W}_{\infty} \rightarrow\left(\mathbb{C}^{*}\right)^{2}$ by

$$
\begin{aligned}
& l_{N, *, c}([x ; p, q]) \\
& \quad=\left(\frac{1}{N}\left(\log (x)+i \pi(N+1)\left(p-* c_{0}\right)\right), \frac{1}{N}\left(\log \left((1-x)^{-1}\right)+i \pi(N+1)\left(q-* c_{1}\right)\right)\right) .
\end{aligned}
$$

Then:

- $\quad \mathbb{C}_{*}\left(\right.$ resp. $\left.\mathcal{W}_{\infty}\right)$ with coordinate $w_{0}$ (resp. $\left.\left[w_{0} ; f_{0}, f_{1}\right]\right)$ is a parametrization of the set of edge decorations $w$ (resp. $(w, f))$ of $(\Delta, b)$.

- Setting $c_{2}=1-c_{0}-c_{1}$, the components of $l_{N, *_{b}, c}\left(\left[w_{0} ; f_{0}, f_{1}\right]\right)$ determine the three quantum log branches $l_{k, N, *_{b}, c}$ of $(\Delta, b, w, f, c)$.

- $\operatorname{Put} \operatorname{EXP}\left(z_{1}, \ldots, z_{2 s}\right)=\left(\exp \left(z_{1}\right), \ldots, \exp \left(z_{2 s}\right)\right), s \geq 1$. The map $\operatorname{EXP} \circ l_{N, *, c}$ : $\mathcal{W}_{\infty} \rightarrow\left(\mathbb{C}^{*}\right)^{2}$ provides a distinguished system of $N^{\text {th }}$ roots of the cross ratios $w_{k}$ :

$$
w_{k}^{\prime}:=\exp \left(l_{k, N, *_{b}, c}\left(\left[w_{0} ; f_{0}, f_{1}\right]\right)\right)
$$


The proof of this lemma is straightforward; see [4] and [5, Remarks 2.1 and 2.18].

Lemma 3.1 (1) The $N^{\text {th }}$ roots $\left(w_{0}^{\prime}, w_{1}^{\prime}, w_{2}^{\prime}\right)$ depend only on the remainders mod $N$ of the flattening $f$ and the charge $c$, and satisfy the relation

$$
w_{0}^{\prime} w_{1}^{\prime} w_{2}^{\prime}=-\zeta^{*} b^{m}
$$

where as usual $N:=2 m+1$ and $\zeta:=\exp (2 i \pi / N)$.

(2) For any charge $c$ and $N^{\text {th }}$ roots $u_{k}$ of the cross ratios $w_{k}$ satisfying the relation (15), there is a flattening $f$ such that $u_{k}=w_{k}^{\prime}$ as in (14).

(3) The image $\mathcal{W}_{N}$ of $\mathcal{W}_{\infty}$ via the map $\operatorname{EXP} \circ l_{N, *_{b}, c}$ is the curve in $\left(\mathbb{C}^{*}\right)^{2}$ with defining equation

$$
u_{0}^{N}+\left(u_{1}^{-1}\right)^{N}=1 .
$$

(4) There is a natural $N^{2}$-to-one rational regular map

$$
p_{N}: \mathcal{W}_{N} \rightarrow \mathbb{C}_{*}, \quad p_{N}\left(u_{0}, u_{1}\right):=u_{0}^{N} .
$$

3A2 Tetrahedral tensors Given a branched tetrahedron $(\Delta, b)$ and an operator $A \in \operatorname{End}\left(\mathbb{C}^{N} \otimes \mathbb{C}^{N}\right)$, associate a copy $V_{j}$ of $\mathbb{C}^{N}$ to the 2 -face $F_{j}$, and consider $A$ as a map

$$
A= \begin{cases}\left(A_{k, l}^{i, j}\right): V_{3} \otimes V_{1} \rightarrow V_{2} \otimes V_{0} & \text { if } *_{b}=+1, \\ \left(A_{i, j}^{k, l}\right): V_{2} \otimes V_{0} \rightarrow V_{3} \otimes V_{1} & \text { if } *_{b}=-1 .\end{cases}
$$

Note that the pair of indices $(i, j)$ (resp. $(k, l))$ corresponds to the space $V_{2} \otimes V_{0}$ (resp. $V_{3} \otimes V_{1}$ ), and to the outgoing (resp. ingoing) 2-face coorientations induced by $b$ if $*_{b}=1$, and the converse if $*_{b}=-1$. The entries $A_{k, l}^{i, j}$ and $A_{i, j}^{k, l}$ are taken in the standard basis of $\mathbb{C}^{N} \otimes \mathbb{C}^{N}$. Graphically this is encoded in Figure 9 (compare with Figure 4).
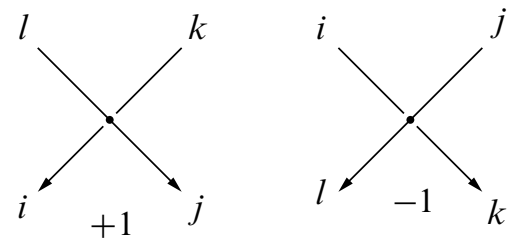

Figure 9: Position of tensor indices

Using (16) we define the tetrahedral tensors $\mathrm{R}_{N}(\Delta, b, w, f, c)$ of a quantum hyperbolic 3-simplex by

$$
\begin{aligned}
\mathcal{R}_{1}(\Delta, b, w, f):=\mathcal{R}_{\left(1, *_{b}\right)}\left(\left[w_{0} ; f_{0}, f_{1}\right]\right) & & \text { if } N=1, \\
\mathcal{R}_{N}(\Delta, b, w, f, c):=\mathcal{R}_{\left(N, *_{b}, c\right)} \circ \operatorname{EXP} \circ l_{N, *_{b}, c}\left(\left[w_{0} ; f_{0}, f_{1}\right]\right) & & \text { if } N \geq 3 \text { is odd, }
\end{aligned}
$$


where $\mathcal{R}_{(1, *)}: \mathcal{W}_{\infty} \rightarrow \mathrm{GL}(\mathbb{C} \otimes \mathbb{C}) \cong \mathbb{C}^{*}$ and $\mathcal{R}_{(N, *, c)}: \mathcal{W}_{N} \rightarrow \mathrm{GL}\left(\mathbb{C}^{N} \otimes \mathbb{C}^{N}\right)$ are defined as follows. The map $\mathcal{R}_{(1, *)}$ is analytic. It is given by

$$
\mathcal{R}_{(1, *)}([x ; p, q]):=\exp \left(* \frac{2}{i \pi}\left(\mathrm{L}(x)+\frac{i \pi}{2}(p \log (1-x)+q \log (x))\right)\right),
$$

where $\mathrm{L}$ is the Rogers dilogarithm

$$
\mathrm{L}(x)=-\frac{\pi^{2}}{6}-\frac{1}{2} \int_{0}^{x} \frac{\log (t)}{1-t}+\frac{\log (1-t)}{t} d t .
$$

The maps $\mathcal{R}_{(N, *, c)}$ are regular rational maps called matrix dilogarithms. They verify noncommutative versions of the five term relations satisfied by L. For every $x \in \mathbb{C}^{*}$, put $x^{1 / N}:=\exp (\log (x) / N)$, extended to $0^{1 / N}:=0$ by continuity. Set

$$
[x]:=N^{-1} \frac{1-x^{N}}{1-x}, \quad g(x):=\prod_{j=1}^{N-1}\left(1-x \zeta^{-j}\right)^{j / N}, \quad h(x):=g(x) / g(1) .
$$

Let $\mathcal{F}_{N}=\left\{\left(u^{\prime}, v^{\prime}\right) \in \mathbb{C}^{2} \mid\left(u^{\prime}\right)^{N}+\left(v^{\prime}\right)^{N}=1\right\}$, and $\omega: \mathcal{F}_{N} \times \mathbb{Z} / N \mathbb{Z} \rightarrow \mathbb{C}$ be the regular rational map given by $\omega\left(u^{\prime}, v^{\prime} \mid 0\right):=1$ and $\omega\left(u^{\prime}, v^{\prime} \mid n\right):=\prod_{j=1}^{[n]_{N}} v^{\prime}\left(1-u^{\prime} \zeta^{j}\right)^{-1}$. Define $\mathcal{L}_{N}: \mathcal{F}_{N} \rightarrow \operatorname{Aut}\left(\mathbb{C}^{N} \otimes \mathbb{C}^{N}\right)$ by (recall that $\left.m=(N-1) / 2\right)$

$$
\mathcal{L}_{N}\left(u^{\prime}, v^{\prime}\right)_{k, l}^{i, j}=h\left(u^{\prime}\right) \zeta^{k j+(m+1) k^{2}} \omega\left(u^{\prime}, v^{\prime} \mid i-k\right) \delta_{N}(i+j-l) .
$$

We have

$$
\left(\mathcal{L}_{N}\left(u^{\prime}, v^{\prime}\right)^{-1}\right)_{i, j}^{k, l}=\frac{\left[u^{\prime}\right]}{h\left(u^{\prime}\right)} \zeta^{-k j-(m+1) k^{2}} \frac{\delta_{N}(i+j-l)}{\omega\left(u^{\prime} / \zeta, v^{\prime} \mid i-k\right)}
$$

Define

$$
\begin{aligned}
& \mathcal{R}_{(N,+1, c)}\left(u_{0}, u_{1}\right)_{k, l}^{i, j}:=\left(u_{0}^{-c_{1}} u_{1}^{c_{0}}\right)^{(N-1) / 2} \mathcal{L}_{N}\left(u_{0}, u_{1}^{-1}\right)_{k, l}^{i, j}, \\
& \mathcal{R}_{(N,-1, c)}\left(u_{0}, u_{1}\right)_{i, j}^{k, l}:=\left(u_{0}^{-c_{1}} u_{1}^{c_{0}}\right)^{(N-1) / 2}\left(\mathcal{L}_{N}\left(u_{0}, u_{1}^{-1}\right)^{-1}\right)_{i, j}^{k, l} .
\end{aligned}
$$

Remark 3.2 The map $\mathcal{R}_{(1, *)}$ does not depend on the charge $c$ and $\mathcal{R}_{(N, *, c)}$ depends on $c$ only by the factor $\left(u_{0}^{-c_{1}} u_{1}^{c_{0}}\right)^{(N-1) / 2}$, but $\mathcal{R}_{N}$ depends on $c$ also via the $\operatorname{map} l_{N, *_{b}, c}$.

Definition 3.3 The local analytic configurations over $(\Delta, b, c)$ are the family of spaces and maps indexed by the odd integers $N \geq 3$, given by

$$
\mathcal{A}_{N}(\Delta, b, c):=\left\{\mathcal{W}_{\infty}, \mathcal{W}_{N}, p_{\infty}, p_{N}, l_{N, *_{b}, c}, \mathcal{R}_{\left(1, *_{b}\right)}, \mathcal{R}_{\left(N, *_{b}, c\right)}\right\}
$$




\section{B Globalization}

In addition to the tetrahedral tensors $\mathrm{R}_{N}$, when dealing with arbitrary triangulations we need also $f$ ace tensors $\mathcal{Q}_{N}$, defined as follows. For every odd $N \geq 3$, consider the symmetric $N \times N$ matrices $S$ and $T$ with entries

$$
S_{j}^{i}=N^{-1 / 2} \zeta^{i j}, \quad T_{j}^{i}=\zeta^{i^{2}(m+1)} \delta_{N}(i+j) .
$$

We show in Lemma 7.3 that $S$ and $T$ generate an $N$-dimensional projective representation of $\operatorname{SL}(2, \mathbb{Z})$. We put $\mathcal{Q}_{N}:=S \cdot T^{-1}$; see Section 7A for the $\cdot$ notation. This $N \times N$ matrix has entries

$$
\left(\mathcal{Q}_{N}\right)_{j}^{i}=\sum_{k=0}^{N-1} S_{j}^{k}\left(T^{-1}\right)_{k}^{i}=N^{-1 / 2} \zeta^{-i j-(m+1) j^{2}},
$$

and is projectively of order 3 , namely $\mathcal{Q}_{N}^{3}=\phi_{N}^{-1} \mathrm{I}_{N}$ with $\phi_{N}=\left(\frac{m+1}{N}\right)$ if $N \equiv 1$ $\bmod 4$, and $\phi_{N}=i\left(\frac{m+1}{N}\right)$ if $N \equiv 3 \bmod 4$.

Let $(T, \tilde{b})$ be a weakly branched triangulation of $\widehat{V}$ made of $s$ branched tetrahedra $\left(\Delta_{j}, b_{j}\right)$. For every collection of charges $c^{j}$ on the $\Delta_{j}$ and every point

$$
[w ; f]:=\left(\left[w_{0}^{1} ; f_{0}^{1}, f_{1}^{1}\right], \ldots,\left[w_{0}^{s} ; f_{0}^{s}, f_{1}^{s}\right]\right) \in \mathcal{W}_{\infty}^{s},
$$

we call $c$ a rough global charge and call $(T, \tilde{b}, w, f, c)$ a rough $Q H$ triangulation. Working dually, consider the oriented graph $\mathcal{N}$ associated to $(T, \widetilde{b})$ as before Lemma 2.9. For every odd $N \geq 1$ define a $Q H$ tensor network over $\mathcal{N}$ as follows:

- Associate $\mathrm{R}_{N}\left(\Delta_{j}, b_{j}, w^{j}, f^{j}, c^{j}\right)$ to the structured vertex $v_{j}$ of $\mathcal{N}$ dual to $\left(\Delta_{j}, b_{j}\right)$.

- If $N \geq 3$, associate $\mathcal{Q}_{N}^{r(e)}$ to each edge $e$ of $\mathcal{N}$ with $\mathbb{Z} / 3 \mathbb{Z}$-color $r(e)$, so the domain and target of the linear map associated to $\mathcal{Q}_{N}^{r(e)}$ correspond respectively to the initial and final endpoints of $e$; if $N=1$, replace $\mathcal{Q}_{N}^{r(e)}$ by the scalar $1 \in \mathbb{C}^{*}$.

Next we define a normalization factor $a_{N}(T, \tilde{b})$. Formally put $a_{1}(T, \tilde{b})=1$. Denote by $q(T, \tilde{b})$ the number of edges $e$ of $\operatorname{Sing}(P)$ such that $r(e)=2 \in \mathbb{Z} / 3 \mathbb{Z}$. Fix an auxiliary orientation on every edge $e$ of $T$. Let $n_{+}(e)$ (resp. $\left.n_{-}(e)\right)$ be the number of abstract diagonal edges $E \rightarrow e$ such that the $\widetilde{b}$-orientation of $E$ agrees (resp. does not agree) with the orientation of $e$. By Lemma $2.7, n_{+}(e)-n_{-}(e)$ is an even integer. Set $\epsilon_{N}:=(-1)^{(N-1) / 2}$. For every odd $N \geq 3$ define

$$
a_{N}(T, \tilde{b}):=N^{-v} \phi_{N}^{-q(T, \tilde{b})} c_{N}(T, \tilde{b}), \quad c_{N}(T, \tilde{b}):=\epsilon_{N}^{v+l-\frac{1}{2} \sum_{e}\left(n_{+}(e)-n_{-}(e)\right)},
$$


where $\phi_{N}$ is as above, and $l, v$ are respectively the number of edges and the number of vertices of $T$ which are manifold points.

Remark 3.4 Clearly $c_{N}(T, \tilde{b})$ does not depend on the choice of the auxiliary edge orientations, and $c_{N}(T, \widetilde{b})=1$ if $N-1 \equiv 0 \bmod 4$. If $\tilde{b}$ is a branching $b$, using the edge $b$-orientation we see that $l-\frac{1}{2} \sum_{e}\left(n_{+}(e)-n_{-}(e)\right)=l-t=\chi(\bar{V})=\chi(P)=-v$, where $\chi$ denotes the Euler characteristic and $t$ the number of tetrahedra of $T$. Hence in this case $c_{N}(T, b)=1$, and $a_{N}(T, b)=N^{-v}$.

A $N$-state of $\mathcal{N}$ is an assignment of a label in $\{0, \ldots, N-1\}$ to each edge; every $N-$ state $\sigma$ determines a matrix element, denoted by $\mathrm{R}_{N}\left(\Delta_{j}, b_{j}, w^{j}, f^{j}, c^{j}\right)_{\sigma}$ or $\mathcal{Q}_{N, \sigma}^{r(e)}$, of each tensor of the $\mathrm{QH}$ tensor network.

Definition 3.5 The $Q H$ state sum function $\mathcal{H}_{N}(T, \tilde{b}, c): \mathcal{W}_{\infty}^{s} \rightarrow \mathbb{C}$ is the total contraction of the $\mathrm{QH}$ tensor network over $\mathcal{N}$, normalized by $a_{N}(T, \tilde{b})$ :

$$
\mathcal{H}_{N}(T, \tilde{b}, c)([w ; f]):=a_{N}(T, \tilde{b}) \sum_{\sigma} \prod_{j} \mathrm{R}_{N}\left(\Delta_{j}, b_{j}, w^{j}, f^{j}, c^{j}\right)_{\sigma} \prod_{e} \mathcal{Q}_{N, \sigma}^{r(e)}
$$

Sometimes we denote $\mathcal{H}_{N}(T, \tilde{b}, c)([w ; f])$ by $\mathcal{H}_{N}(\mathcal{T})$, where $\mathcal{T}:=(T, \tilde{b}, w, f, c)$, and when what is meant is clear from context, $\mathcal{H}_{N}(T, \tilde{b}, c)$ by $\mathcal{H}_{N}$. Note that $\mathcal{H}_{1}$ is a product of analytic scalar functions and does not depend on $c$. When $N \geq 3$, $\mathcal{H}_{N}(T, \tilde{b}, c)=\mathcal{H}_{N}^{\prime}(T, \tilde{b}, c) \circ\left(\operatorname{EXP} \circ l_{N, *_{b}, c}\right)$, where $\mathcal{H}_{N}^{\prime}(T, \tilde{b}, c)$ is a rational regular function defined on the algebraic variety $\mathcal{W}_{N}^{s}$.

In order to make $(21)$ an actual invariant state sum, we need to restrict $[w ; f] \in \mathcal{W}_{\infty}^{s}$ to a suitable subspace, which we denote by $G_{0}(T, \widetilde{b})_{\infty}$ below (later we will do similarly for charges, see Definition 4.7 and 5.1). Consider the model $G(T, \tilde{b})$ of the gluing variety and the products of the maps of Section 3 A over the $\Delta_{j}$ (keeping, with slight abuse, the same notations):

$$
\begin{aligned}
p_{\infty}: \mathcal{W}_{\infty}^{s} \rightarrow \mathbb{C}_{*}^{s}, & p_{N}: \mathcal{W}_{N}^{s} \rightarrow \mathbb{C}_{*}^{s}, \\
l_{N, *, c}: \mathcal{W}_{\infty}^{s} \rightarrow\left(\mathbb{C}^{*}\right)^{2 s}, & \operatorname{EXP} \circ l_{N, *, c}: \mathcal{W}_{\infty}^{s} \rightarrow \mathcal{W}_{N}^{s} \subset\left(\mathbb{C}^{*}\right)^{2 s} .
\end{aligned}
$$

Set

$$
G(T, \tilde{b})_{\infty}:=p_{\infty}^{-1}(G(T, \tilde{b})) \quad \text { and } \quad G(T, \tilde{b}, c)_{N}:=\operatorname{EXP}_{\infty} l_{N, *_{b}, c}\left(G(T, \tilde{b})_{\infty}\right)
$$

The restrictions of $p_{\infty}$ and $p_{N}$ to these spaces are covering maps of $G(T, \tilde{b})$, that make a commutative diagram with $\operatorname{EXP} \circ l_{N, *_{b}, c} ; G(T, \tilde{b})_{\infty}$ is a closed analytic subset of $\mathcal{W}_{\infty}^{s}, G(T, \tilde{b}, c)_{N}$ an algebraic subset of $\mathcal{W}_{N}^{s}$, and $\operatorname{Sing}\left(G(T, \tilde{b})_{*}\right)=$ $p_{*}^{-1}(\operatorname{Sing}(G(T, \tilde{b}))$. 
Define the total cross ratio of an edge $e$ of $T$ by (see the notations at the end of Section 2)

$$
W(e):=\prod_{E \rightarrow e} w(E)^{*} E
$$

so that $[w ; f] \in G(T, \tilde{b})_{\infty}$ if and only if $W(e)=1$ for all $e$. Similarly, define the total log branch, total $N^{\text {th }}$ root modulus, and total charge at $e$ by

$$
L(e):=\sum_{E \rightarrow e} *_{E} l(E), \quad W_{N}^{\prime}(e):=\prod_{E \rightarrow e} w^{\prime}(E)^{*}, \quad C(e):=\sum_{E \rightarrow e} c(E),
$$

where $l(E)=\log \left(w_{k}^{j}\right)+i f_{k}^{j} \pi, w(E)=w_{k}^{j}$ and $c(E)=c_{k}^{j}$ if and only if $E$ is an edge of $\left(\Delta_{j}, b_{j}, w^{j}\right), E$ is either $E_{k}^{j}$ or the opposite edge, and $*_{E}=*_{b_{j}}$. Fix an auxiliary ordering of the, say $n$, edges of $(T, \tilde{b})$. We have analytic maps

$$
\begin{aligned}
& t_{L}: \mathcal{W}_{\infty}^{s} \rightarrow \mathbb{C}^{n}, t_{L}([w ; f]):=\left(L\left(e_{1}\right), \ldots L\left(e_{n}\right)\right), \\
& t_{N, W^{\prime}, c}: \mathcal{W}_{\infty}^{s} \rightarrow \mathbb{C}^{n}, \quad t_{N, W^{\prime}, c}([w ; f]):=\left(W_{N}^{\prime}\left(e_{1}\right), \ldots W_{N}^{\prime}\left(e_{s}\right)\right),
\end{aligned}
$$

the dependence of $L\left(e_{k}\right)$ (resp. $\left.W_{N}^{\prime}\left(e_{k}\right)\right)$ on $[w ; f]$ (resp. $[w ; f]$ and $c$ ) being understood. The following lemma is evident.

Lemma 3.6 The restriction of $t_{L}$ to $G(T, \tilde{b})_{\infty}$ takes values in $(2 i \pi \mathbb{Z})^{n}$ and the restriction of $t_{N, W^{\prime}, c}$ takes values in the set $\mu_{N}$ of $N^{\text {th }}$ roots of unity. Both maps are discrete-valued, hence constant on the closure of each connected component of the set of nonsingular points of $G(T, \widetilde{b})_{\infty}$. Moreover, the restriction of $t_{N, W^{\prime}, c}$ factorizes as

$$
t_{N, W^{\prime}, c}=t_{N, W}^{\prime} \circ\left(\mathrm{EXP} \circ l_{N, *_{b}, c}\right)
$$

where $t_{N, W}^{\prime}$ is a rational regular map defined on $G(T, \tilde{b}, c)_{N}$ and constant on irreducible components.

Finally define the analytic subset

$$
G_{0}(T, \tilde{b})_{\infty}=t_{L}^{-1}(0, \ldots, 0) \subset G(T, \tilde{b})_{\infty}
$$

and, for every $N$, the algebraic subvariety

$$
G_{0}(T, \tilde{b}, c)_{N}=\operatorname{EXP} \circ l_{N, *_{b}, c}\left(G_{0}(T, \tilde{b})_{\infty}\right) \subset G(T, \tilde{b}, c)_{N}
$$

As usual, keep the same notation for the restriction of any map already defined.

Definition 3.7 The $N^{\text {th }}$ analytic configuration over $(T, \tilde{b}, c)$ is the family of spaces and maps

$$
\mathcal{A}_{N}(T, \tilde{b}, c):=\left\{G_{0}(T, \tilde{b})_{\infty}, G_{0}(T, \tilde{b}, c)_{N}, p_{\infty}, p_{N}, l_{N, *_{b}, c}, \mathcal{H}_{1}, \mathcal{H}_{N}\right\}
$$


We stress that every map $t_{N, W^{\prime}, c}$ is constant on $G_{0}(T, \tilde{b})_{\infty}$, and its value depends on the fixed rough charge $c$, precisely on the total edge charges $\left(C\left(e_{1}\right), \ldots, C\left(e_{n}\right)\right)$.

\section{Cusped manifolds}

Let $M$ be a cusped manifold, and $V$ be as in Section 1A, that is, a compact oriented connected 3-manifold with one torus boundary component such that $M$ is diffeomorphic to the interior of $V$. We use the notation $\widehat{V}$ as in Section 2.

\section{A The augmented PSL $(2, \mathbb{C})$-character variety and $A$-polynomial}

Fix a geometric basis $(l, m)$ of the fundamental group $\pi_{1}(\partial V) \cong \mathbb{Z} \times \mathbb{Z}$. It is given by a couple of oriented simple closed curves on $\partial V$ which meet at one point, transversely and positively. If $M$ is the complement of a hyperbolic knot $K$ in $S^{3}$ we can take a canonical longitude $l$ and a meridian $m$ of $K$.

There is a conjugacy class $[\sigma]$ of representations of $\pi_{1}(\partial V)$ in $\operatorname{PSL}(2, \mathbb{C})$, each one with images isomorphic to $\mathbb{Z} / 2 \mathbb{Z} \times \mathbb{Z} / 2 \mathbb{Z}$, acting on the Riemann sphere $\mathbb{C P}^{1}=\partial \mathbb{H}^{3}$ without a common fixed point. The class $[\sigma]$ is obtained as follows: pick two geodesic lines in $\mathbb{H}^{3}$, say $\gamma_{l}$ and $\gamma_{m}$, that meet at one point forming a right angle; then define $\sigma$ by $\sigma(x)=r_{x}$, where $x \in\{l, m\}$ and $r_{x}$ is the rotation by $\pi$ around $\gamma_{x}$.

By definition, the $\operatorname{PSL}(2, \mathbb{C})$-character variety of $M$ is the algebro-geometric quotient

$$
X^{\prime}(M):=\operatorname{Hom}^{\prime}\left(\pi_{1}(M), \operatorname{PSL}(2, \mathbb{C})\right) / / \operatorname{PSL}(2, \mathbb{C}),
$$

where $\operatorname{PSL}(2, \mathbb{C})$ acts by conjugation, and

$$
\operatorname{Hom}^{\prime}\left(\pi_{1}(M), \operatorname{PSL}(2, \mathbb{C})\right) \subset \operatorname{Hom}\left(\pi_{1}(M), \operatorname{PSL}(2, \mathbb{C})\right)
$$

is the subset of representations that do not restrict to an element of $[\sigma]$ on $\pi_{1}(\partial V)$. Given a peripheral subgroup $\pi_{1}(\partial V)$ of $\pi_{1}(M) \cong \pi_{1}(V)$, denote by

$$
R(M) \subset \operatorname{Hom}\left(\pi_{1}(V), \operatorname{PSL}(2, \mathbb{C})\right) \times \mathbb{C P}^{1}
$$

the set of couples $(r, z)$ such that $z$ is fixed by $r\left(\pi_{1}(\partial V)\right)$. In particular, couples such that $[r]$ restricts to $[\sigma]$ on $\partial V$ are excluded. The augmented $\operatorname{PSL}(2, \mathbb{C})$-character variety of $M$ is the algebro-geometric quotient

$$
X(M):=R(M) / / \operatorname{PSL}(2, \mathbb{C}),
$$

where PSL $(2, \mathbb{C})$ acts by conjugation on $R(M)$ and by Möbius transformations on $\mathbb{C P}^{1}$. Hence, any augmented character of $M, \rho=[(\rho, z)] \in X(M)$, is a character 
of representations $r: \pi_{1}(M) \rightarrow \operatorname{PSL}(2, \mathbb{C})$ together with a choice of fixed points of the peripheral subgroups, invariant by conjugation. The hyperbolic holonomy of $M$ defines a point $\rho_{\text {hyp }} \in X(M)$. In a similar way we can define the $\operatorname{PSL}(2, \mathbb{C})$-character variety $X^{\prime}(\partial V)$ of $\partial V$ and its augmented version $X(\partial V)$. The inclusion $\iota: \partial V \rightarrow V$ induces regular maps $\iota^{*}: X^{\prime}(M) \rightarrow X^{\prime}(\partial V)$ and $\iota^{*}: X(M) \rightarrow X(\partial V)$.

Theorem 4.1 (1) ([10; 16]; see also [35]) Both $X^{\prime}(M)$ and $X(M)$ are complex algebraic affine varieties, and the natural projection $q: X(M) \rightarrow X^{\prime}(M)$ is a regular map.

(2) [22] $\rho_{\text {hyp }}\left(\right.$ resp. $q\left(\rho_{\text {hyp }}\right)$ ) is a regular point of $X(M)$ (resp. $\left.X^{\prime}(M)\right)$. Hence it belongs to a unique irreducible component $X_{0}(M)$ of $X(M)$ (resp. $X_{0}^{\prime}(M)$ of $\left.X^{\prime}(M)\right)$, which is a complex algebraic curve.

(3) The restriction of $q$ to $X_{0}(M), q_{0}: X_{0}(M) \rightarrow X_{0}^{\prime}(M)$, is generically $2: 1$.

(4) [18] The restricted map $\iota^{*}: X_{0}^{\prime}(M) \rightarrow X_{0}^{\prime}(\partial V)\left(\operatorname{resp} . \iota^{*}: X_{0}(M) \rightarrow X_{0}(\partial V)\right)$ is generically one-to-one. Hence it is a birational isomorphism onto its image.

For any $\rho \in X(\partial V)$ which is nontrivial, let $\bar{\rho}$ be a representative of $\rho$ such that $\bar{\rho}\left(\pi_{1}(\partial V)\right)$ fixes $z=\infty \in \mathbb{C P}^{1}$. For any nonzero class $\gamma \in \pi_{1}(\partial V), \bar{\rho}(\gamma)$ acts on $\mathbb{C}$ as $w \mapsto \gamma_{\rho} w+b$, where $\gamma_{\rho} \in \mathbb{C}^{*}$ and $b \in \mathbb{C}$. In general $\gamma_{\rho}$ is a squared eigenvalue of $\bar{\rho}(\gamma) \in \operatorname{PSL}(2, \mathbb{C})$; if $\bar{\rho}(\gamma)$ is loxodromic, then $\gamma_{\rho} \neq 1$, and the two reciprocally inverse eigenvalues are distinguished by the augmentation, which selects an endpoint, whence an orientation, of $\gamma$. Consider the so called holonomy map

$$
\operatorname{hol}_{\gamma}: X(\partial V) \rightarrow \mathbb{C}^{*}, \quad \rho \mapsto \gamma_{\rho} .
$$

By using the above cusp basis $(l, m)$ we get an algebraic isomorphism

$$
\operatorname{hol}_{m} \times \operatorname{hol}_{l}: X(\partial V) \rightarrow \mathbb{C}^{*} \times \mathbb{C}^{*} \text {. }
$$

Define the rational map

$$
\mathfrak{h}: X(M) \rightarrow \mathbb{C}^{*} \times \mathbb{C}^{*}, \quad \mathfrak{h}=\left(\operatorname{hol}_{m} \times \mathrm{hol}_{l}\right) \circ \iota^{*}
$$

Following [12; 14] and Dunfield's appendix in [17], consider the plane curve $A(M)$ defined as the closure of the 1-dimensional part of the image $\mathfrak{h}(X(M))$. The (suitably normalized) polynomial generating the ideal of $A(M)$ is by definition the PSL $(2, \mathbb{C})$ A-polynomial of $M$. We call the closure of $\mathfrak{h}\left(X_{0}(M)\right)$ the geometric component of $A(M)$, and denote it by $A_{0}(M)$. Then Theorem 4.1(4) admits a corollary.

Corollary 4.2 The restricted map $\mathfrak{h}: X_{0}(M) \rightarrow A_{0}(M)$ is a birational isomorphism. 


\section{B Rich components of $G(T)$}

Let $T$ be any ideal triangulation of $\widehat{V}$ such that the gluing variety $G(T)$ is nonempty. There is a natural regular map (see [12] or the appendix of [17])

$$
\rho: G(T) \rightarrow X(M) .
$$

As $G(T)$ is a complex algebraic curve, $\mathfrak{h}(\rho(G(T))$ is a union of irreducible components of the plane curve $A(M)$. Assume that $G(T)$ contains a nonnegative point $z_{h}$ such that $\rho\left(z_{h}\right)=\rho_{\text {hyp }}$. Recall that nonnegative means that for every tetrahedron $\Delta_{j}$ of $T$ the cross ratios $z_{h}^{j}$ have nonnegative imaginary parts. The point $z_{h}$ is not necessarily a regular point of the gluing variety, hence in general it could be contained in several irreducible components of $G(T)$.

Definition 4.3 An irreducible component of $G(T)$ is rich if it contains $z_{h}$ and an infinite sequence of points $z_{n}$ that converge to $z_{h}$ (in the strong topology of $G(T)$ as an analytic space), and correspond to compact closed hyperbolic Dehn fillings of $M$ that converge geometrically to $M$.

Proposition 4.4 For every gluing variety $G(T)$ containing a nonnegative point $z_{h}$ with $\rho\left(z_{h}\right)=\rho_{\text {hyp }}$, the set of rich components of $G(T)$ is nonempty and finite.

Proof This follows from the proof of the hyperbolic Dehn filling theorem given in [34], and the fact that the algebraic curve $G(T)$ has a finite number of irreducible components.

Remark 4.5 In [33], the hyperbolic Dehn filling theorem was proved assuming that $M$ (allowing several cusps) has an ideal triangulation $T$ such that $G(T)$ contains a strictly positive point $z_{h}$ representing the hyperbolic holonomy. This proof also uses that $z_{h}$ is a regular point of $G(T)$. By elaborating on the analysis of [33], the paper [13] has provided a proof of this regularity result, extended to every strictly positive point of $G(T)$. In [7], the proof from [33] was presented with some mild modifications avoiding the assumption that $z_{h}$ is regular. Unfortunately, it did not yet give a complete proof of the hyperbolic Dehn filling theorem based on gluing varieties, since it is not known if every $M$ has strictly positive geometric ideal triangulations. The paper [34] filled this gap; it uses any gluing variety $G(T)$ with a nonnegative and possibly singular point $z_{h}$, which always exists. The proof also uses the arguments of [7]. In [27] it is proved that strictly positive geometric ideal triangulations exist virtually, that is, every cusped $M$ has a finite covering $\tilde{M}$ with such an ideal triangulation. On the other hand, passing to $\tilde{M}$ increases the number of cusps. 
Proposition 4.6 Let $Z$ be any rich component of $G(T)$. Then the restricted maps $\rho: Z \rightarrow X_{0}(M)$ and $\mathfrak{h} \circ \rho: Z \rightarrow A_{0}(M)$ are generically one-to-one and hence are both birational isomorphisms. More precisely, there are maximal nonempty Zariski open subsets $\Omega_{Z}^{0}$ and $\Omega_{Z}$ of $Z$, with $\Omega_{Z}^{0} \subset \Omega_{Z}$, such that:

(1) $\rho\left(\Omega_{Z}\right)$ and $\rho\left(\Omega_{Z}^{0}\right)$ are Zariski open subsets of $X_{0}(M)$ (resp. $\mathfrak{h} \circ \rho\left(\Omega_{Z}\right)$ and $\mathfrak{h} \circ \rho\left(\Omega_{Z}^{0}\right)$ are Zariski open subsets of $\left.A_{0}(M)\right)$.

(2) The restriction of $\rho$ and $\mathfrak{h} \circ \rho$ to $\Omega_{Z}$ is one-to-one onto its image, and $z_{h} \in \Omega_{Z}$. The restriction of $\rho$ and $\mathfrak{h} \circ \rho$ to $\Omega_{Z}^{0}$ is a regular rational isomorphism onto its image.

Proof This goes step by step as the proof of [18, Theorem 3.1]. The key ingredients are the Gromov-Thurston volume rigidity for closed hyperbolic manifolds, the fact that the volume of representations yields a well defined function on the normalization of the smooth projective model of $A_{0}(M)$, and the existence on $X_{0}(M)$ of infinite sequences of points corresponding to the holonomies of compact closed hyperbolic Dehn fillings of $M$ that converge geometrically to $M$. In the present situation this last fact is ensured by the definition of a rich component, and the volume of representations lifts to the function (6) on $G(T)$.

If $T$ has a weak branching $\tilde{b}$, the above discussion can be rephrased in terms of the gluing variety $G(T, \tilde{b})$ of Section 2 . Recall that a system of cross ratios $w^{j}$ on the branched tetrahedra $\left(\Delta_{j}, b_{j}\right)$ of $(T, \tilde{b})$ is nonnegative if $z^{j}=\left(w^{j}\right)^{*_{j}}$ is nonnegative.

\section{C Refined analytic configurations for one-cusped manifolds}

Let $(T, \widetilde{b})$ be a weakly branched ideal triangulation of $\widehat{V}$.

Definition 4.7 A global charge $c$ on $T$ is a rough global charge satisfying the additional global constraint that for every edge $e$ of $T$, the total edge charge $C(e)$ is constant and equal to 2 .

For every global charge $c$ the variety $G_{0}(T, b, c)_{N}$ has at every edge $e$ of $T$ the defining equation

$$
W^{\prime}(e)=\zeta^{-1},
$$

where $\zeta:=\exp (2 \pi i / N)$. Note that $\zeta^{-1}=\zeta^{2 m}$. We have: 
Proposition 4.8 (1) Every global charge $c$ on $(T, \tilde{b})$ determines a $c$-weight $\left(h_{c}, k_{c}\right)$ of $V$.

(2) For every $c$-weight $\left(h_{c}, k_{c}\right)$ of $V$ and every ideal triangulation $(T, \tilde{b})$ of $\hat{V}$, there exists a global charge $c$ on $(T, \tilde{b})$ with $c$-weight equal to $\left(h_{c}, k_{c}\right)$.

Proposition 4.9 Assume the gluing variety $G(T, \tilde{b})$ is nonempty, and let $\rho: G(T, \tilde{b}) \rightarrow$ $X(M)$ be as in (24).

(1) Every point $[w ; f] \in G_{0}(T, \tilde{b})_{\infty}$ determines an $f$-weight $\left(h_{f}, k_{f}\right)$ of $(V, \rho(w))$.

(2) For every point $\rho \in \rho(G(T, \tilde{b}))$ and every $f$-weight $\left(h_{f}, k_{f}\right)$ of $(V, \rho)$, there exists a point $[w ; f]$ of $G_{0}(T, \widetilde{b})_{\infty}$ with $f$-weight equal to $\left(h_{f}, k_{f}\right)$.

Propositions 4.8 and 4.9 are reformulations of results of Neumann [31; 32]. We recall below the constructions underlying the statements (1), which claim the existence of the pairs $\left(\gamma(c), \gamma_{2}^{\prime}(c)\right)$ and $\left(L([w ; f]), \gamma_{2}^{\prime}(f)\right)$ in Definition 4.10. The statements in (2) are much more subtle. For the sake of completeness, we recall the proofs at the end of the section (part of the material will be used in Section 4D).

Denote by $T_{0}$ the cell complex obtained from $T$ by removing an open cone neighborhood of its vertex. Hence $T_{0}$ is the result of gluing tetrahedra with truncated vertices, and the polyhedron underlying $T_{0}$ is homeomorphic to $V$. Denote by $\partial T_{0}$ the induced triangulation of $\partial V$. Represent any nonzero class in $H_{1}(\partial V ; \mathbb{Z})$ by normal loops, that is, a disjoint union of oriented essential simple closed curves in $\partial V$, transverse to the edges of $\partial T_{0}$ and such that no curve enters and exits a $2-$ simplex by a same edge. The intersection of a normal loop, say $C$, with a 2-simplex $F$ consists of a disjoint union of arcs, each of which turns around a vertex of $F$; if $F$ is a cusp section of the tetrahedron $\Delta$ of $T$, for every vertex $v$ of $F$ we denote by $E_{v}$ the edge of $\Delta$ containing $v$, and by $*_{v}$ the branching sign of $\Delta$. We write $C \rightarrow E_{v}$ to mean that some subarcs of $C$ turn around $v$. We count them algebraically, by using the orientation of $C$ : if there are $s_{+}$(resp. $s_{-}$) such subarcs whose orientation is compatible with (resp. opposite to) the orientation of $\partial V$ as viewed from $v$, then we set $\operatorname{ind}(C, v):=s_{+}-s_{-}$. For every point $[w ; f] \in G_{0}(T, \tilde{b})_{\infty}$ and every global charge $c$ on $(T, \tilde{b})$, one defines cohomology classes $L([w ; f]) \in H^{1}(\partial V ; \mathbb{C})$ and $\gamma(c) \in H^{1}(\partial V ; \mathbb{Z})$ by $(C$ is a normal loop on $\partial V)$

$$
\begin{aligned}
L([w ; f])([C]) & :=\sum_{C \rightarrow E_{v}} *_{v} \operatorname{ind}(C, v) l\left(E_{v}\right) \\
& =\sum_{C \rightarrow E_{v}} *_{v} \operatorname{ind}(C, v)\left(\log \left(w\left(E_{v}\right)\right)+\pi i f\left(E_{v}\right)\right), \\
\gamma(c)([C]) & :=\sum_{C \rightarrow E_{v}} \operatorname{ind}(C, v) c\left(E_{v}\right) .
\end{aligned}
$$


Additional classes $\gamma_{2}^{\prime}(c), \gamma_{2}^{\prime}(f) \in H^{1}(V ; \mathbb{Z} / 2 \mathbb{Z})$ are defined similarly, by using normal loops in $T$ and taking the sum mod 2 of the charges or flattenings, respectively, of the edges we face along the loops. The reduction mod 2 of $\gamma(c)$ coincides with the image of $\gamma_{2}^{\prime}(c)$ under the map induced on cohomology by the inclusion $\iota: \partial V \rightarrow V$. Hence $r(\gamma(c))=\iota^{*}\left(\gamma_{2}^{\prime}(c)\right)$. Also, denoting by $d_{w} \in H^{1}(\partial V ; \mathbb{C} / 2 i \pi \mathbb{Z})$ the log of the linear part of the restriction of $\rho(w)$ to $\pi_{1}(\partial V)$, for all $a \in H_{1}(\partial V ; \mathbb{Z})$ we have

$$
\begin{aligned}
L([w ; f])(a) & =d_{w}(a) \bmod i \pi, \\
\left(L([w ; f])(a)-d_{w}(a)\right) / i \pi & =\iota^{*}\left(\gamma_{2}^{\prime}(f)\right)(a) \bmod 2 .
\end{aligned}
$$

This proves that the following definition agrees with the defining constraints of weights (see (1) and (2) in the introduction).

Definition 4.10 The $f$-weight $\left(k_{f}, h_{f}\right)$ of a point $[w ; f] \in G_{0}(T, \tilde{b})_{\infty}$, and the $c-$ weight $\left(k_{c}, h_{c}\right)$ of a global charge $c$ on $(T, \tilde{b})$, are defined respectively as the pairs of cohomology classes

$$
\begin{array}{r}
\left(L([w ; f]), \gamma_{2}^{\prime}(f)\right) \in H^{1}(\partial V ; \mathbb{C}) \times H^{1}(V ; \mathbb{Z} / 2 \mathbb{Z}), \\
\left(\gamma(c), \gamma_{2}^{\prime}(c)\right) \in H^{1}(\partial V ; \mathbb{Z}) \times H^{1}(V ; \mathbb{Z} / 2 \mathbb{Z}) .
\end{array}
$$

Example 4.11 (Example 2.12 continued) Every point $w$ of the gluing variety $G\left(T_{1}, \tilde{b}\right)$ gives rise to a class $\exp \left(d_{w}\right) \in H^{1}\left(\partial V ; \mathbb{C}^{*}\right)$. For a suitable choice of geometric basis $(\lambda, \mu)$ of $H_{1}(\partial V ; \mathbb{Z})$ we have

$$
\exp \left(d_{w}\right)(\lambda)=w_{0} w_{1} W_{0}^{-1} W_{1}^{-1} \quad \text { and } \quad \exp \left(d_{w}\right)(\mu)=w_{1}^{-2} W_{2}^{2} .
$$

Denote by $f_{j}$ and $l_{j}:=\log \left(w_{j}\right)+i \pi f_{j}$ (resp. $F_{j}$ and $L_{j}:=\log \left(W_{j}\right)+i \pi F_{j}$ ) the flattening and $\log$ branch variables at the top (resp. bottom) crossing of Figure 7 . The equations for the $\log$ branch variables are (see (9))

$$
\begin{aligned}
l_{0}+l_{1}+l_{2}=L_{0}+L_{1}+L_{2} & =0, \\
l_{0}+2 l_{1}+L_{0}+2 L_{1} & =0, \\
l_{0}+2 l_{2}+L_{0}+2 L_{2} & =0 .
\end{aligned}
$$

For simplicity let us deal with the positive points $w$ of the (unique) rich component of $G\left(T_{1}, \widetilde{b}\right)$ which contains the hyperbolic solution, $w_{1}=W_{1}=\exp (i \pi / 3)$. For every such a positive point the equations for the flattening variables are

$$
\begin{array}{r}
f_{0}+f_{1}+f_{2}=F_{0}+F_{1}+F_{2}=-1, \\
f_{0}+2 f_{1}+F_{0}+2 F_{1}=-2, \\
f_{0}+2 f_{2}+F_{0}+2 F_{2}=-2 .
\end{array}
$$


Indeed, under positivity of $w$ the pairs of first two equations in the systems (30) and (29) are clearly equivalent to each other. Although a bit subtler, this is true also for the remaining pairs of equations, because the consistency relations (9) imply that the sum of the arguments of the cross ratios around edges is exactly equal to $2 \pi$; see [7, Lemma E.6.1].

The equations for the charge variables are formally obtained from those for the flattening ones by replacing in (30) each $f_{j}$ with $-c_{j}$ and each $F_{j}$ with $-C_{j}$. Solving the systems, we get

$$
\begin{array}{lll}
f_{0}=-\left(2 f_{1}+F_{1}-F_{2}+1\right), & F_{0}=-\left(F_{1}+F_{2}+1\right), & f_{2}=f_{1}+F_{1}-F_{2}, \\
c_{0}=-\left(2 c_{1}+C_{1}-C_{2}-1\right), & C_{0}=-\left(C_{1}+C_{2}-1\right), & c_{2}=c_{1}+C_{1}-C_{2}, \\
l_{0}=-\left(2 l_{1}+L_{1}-L_{2}\right), & L_{0}=-\left(L_{1}+L_{2}\right), & l_{2}=l_{1}+L_{1}-L_{2} .
\end{array}
$$

Let us turn to the boundary $c$-weight $k_{c} \in H^{1}(\partial V ; \mathbb{Z})$ and, for every positive $w$ as above, the boundary $f$-weight $k_{f}=k_{f}(w) \in H^{1}(\partial V ; \mathbb{C})$. They are given by

$$
\begin{array}{ll}
k_{c}(\lambda)=c_{0}+c_{1}-C_{0}-C_{1}, & k_{c}(\mu)=2 C_{2}-2 c_{1}, \\
k_{f}(\lambda)=l_{0}+l_{1}-L_{0}-L_{1}, & k_{f}(\mu)=2 L_{2}-2 l_{1} .
\end{array}
$$

At the complete solution we have

$$
k_{f}(\lambda) / i \pi=f_{0}+f_{1}-F_{0}-F_{1}, \quad k_{f}(\mu) / i \pi=2 F_{2}-2 f_{1} .
$$

Hence $k_{f} / i \pi \in H^{1}(\partial V ; \mathbb{Z})$, and $k_{f}(\mu) / i \pi=0 \bmod 2$. Moreover

$$
\begin{array}{ll}
f_{0}=k_{f}(\lambda) / i \pi-k_{f}(\mu) / 2 i \pi-2 f_{1}-1, & F_{0}=k_{f}(\lambda) / i \pi-3 k_{f}(\mu) / 2 i \pi-2 f_{1}-1, \\
& F_{1}=k_{f}(\mu) / i \pi-k_{f}(\lambda) / i \pi+f_{1}, \\
f_{2}=f_{1}+k_{f}(\mu) / 2 i \pi-k_{f}(\lambda) / i \pi, & F_{2}=f_{1}+k_{f}(\mu) / 2 i \pi .
\end{array}
$$

Hence for any given boundary $f$-weight $k_{f}$, there is a one-parameter family of flattenings that realize $k_{f}$, depending on the free parameter $f_{1}$. Similarly for the charges we have

$$
\begin{array}{ll}
c_{0}=k_{c}(\lambda)-k_{c}(\mu) / 2-2 c_{1}+1, & C_{0}=k_{c}(\lambda)-3 k_{c}(\mu) / 2-2 c_{1}+1, \\
& C_{1}=k_{c}(\mu)-k_{c}(\lambda)+c_{1}, \\
c_{2}=c_{1}+k_{c}(\mu) / 2-k_{c}(\lambda), & C_{2}=c_{1}+k_{c}(\mu) / 2,
\end{array}
$$


where the free parameter is now $c_{1}$. By taking $f_{1}=c_{1}=0$, the relevant pairs of parameters that enter the formulas of the tensors $\mathcal{R}_{N}$ are

$$
\begin{aligned}
\left(f_{0}, f_{1}\right) & =\left(k_{f}(\lambda) / i \pi-1-k_{f}(\mu) / 2 i \pi, 0\right), \\
\left(F_{0}, F_{1}\right) & =\left(-1+k_{f}(\lambda) / i \pi-3 k_{f}(\mu) / 2 i \pi, k_{f}(\mu) / i \pi-k_{f}(\lambda) / i \pi\right), \\
\left(c_{0}, c_{1}\right) & =\left(k_{c}(\lambda)+1-k_{c}(\mu) / 2,0\right), \\
\left(C_{0}, C_{1}\right) & =\left(1+k_{c}(\lambda)-3 k_{c}(\mu) / 2, k_{c}(\mu)-k_{c}(\lambda)\right),
\end{aligned}
$$

stressing in this way a pure dependence on the weights. Finally, the state sums are $\mathcal{H}_{N}\left(T_{1}, \tilde{b}, w, W, f, F, c, C\right)$

$$
=\sum_{i, j, l, k, I, J=0}^{N-1} \mathcal{R}_{N}(1, w, f, c)_{k, l}^{i, j} \mathcal{R}_{N}(1, W, F, C)_{j, i}^{I, J}\left(\mathcal{Q}^{2}\right)_{I}^{l}(\mathcal{Q})_{J}^{k},
$$

where we quoted that $*_{b}=1$ for both tetrahedra.

Now let $Z$ be a rich component of $G(T, \tilde{b})$. Denote $Z_{\infty}:=p_{\infty}^{-1}(Z)$ (an analytic subspace of $\left.G_{0}(T, \tilde{b})_{\infty}\right)$, and let $Z_{\infty, 0}$ be the union of connected components of $Z_{\infty}$ made of points $[w ; f]$ such that

$$
\iota^{*}\left(\gamma_{2}^{\prime}(f)\right)=0 \in H^{1}(\partial V ; \mathbb{Z} / 2 \mathbb{Z}) .
$$

Recall the basis $(l, m)$ of $\pi_{1}(\partial V)$. Because of (28), for every point $[w ; f] \in Z_{\infty, 0}$,

$$
\left(e^{L([w ; f])(l)}, e^{L([w ; f])(m)}\right)=\mathfrak{h} \circ \rho(w),
$$

and for every global charge $c$ on $(T, \tilde{b})$, the formula

$$
\begin{aligned}
& L_{N, c}([w ; f])(C) \\
& \quad:=\sum_{C \rightarrow E_{v}} *_{v} \operatorname{ind}(C, v) l_{N, *_{v}, c}\left(E_{v}\right) \\
& =\sum_{C \rightarrow E_{v}} \frac{*_{v}}{N} \operatorname{ind}(C, v)\left(\log \left(w\left(E_{v}\right)\right)+\pi i(N+1)\left(f\left(E_{v}\right)-*_{v} c\left(E_{v}\right)\right)\right) \\
& =\frac{1}{N}(L([w ; f])([C])-\pi i(N+1) \gamma(c)([C]))+\pi i \underbrace{\sum_{C \rightarrow E_{v}} *_{v} \operatorname{ind}(C, v) f\left(E_{v}\right)}_{\in 2 \mathbb{Z}}
\end{aligned}
$$

yields a cohomology class $L_{N, c}([w ; f]) \in H^{1}(\partial V ; \mathbb{C} / 2 \pi i \mathbb{Z})$ such that

$$
\left(\left(e^{L_{N, c}([w ; f])(l)}\right)^{N},\left(e^{L_{N, c}([w ; f])(m)}\right)^{N}\right)=\mathfrak{h} \circ \rho(w) .
$$


If we allow [w;f] to live in $Z_{\infty} \backslash Z_{\infty, 0}$, the sum in (33) can be odd, and the equalities (32) and (34) hold true only up to signs in each component. More precisely, for all $[w ; f] \in Z_{\infty}$ the sum in (33) is an integer expression of the residue class $\gamma_{2}^{\prime}(f)([C]) \in$ $\mathbb{Z} / 2 \mathbb{Z}$, so that we have a well-defined cohomology class

$$
L_{N, c}([w ; f]) \in H^{1}(\partial V ; \mathbb{C} / 2 \pi i \mathbb{Z})
$$

such that

$$
L_{N, c}([w ; f])=\frac{1}{N}(L([w ; f])-\pi i(N+1) \gamma(c))+\pi i \iota^{*}\left(\gamma_{2}^{\prime}(f)\right) \bmod 2 \pi i \mathbb{Z},
$$

where, as usual, $\iota^{*}: H^{1}(V ; \mathbb{Z} / 2 \mathbb{Z}) \rightarrow H^{1}(\partial V ; \mathbb{Z} / 2 \mathbb{Z})$ is induced by the inclusion map $\iota: \partial V \rightarrow V$. The spaces $Z_{\infty, 0}$ and $A_{0}(M)$ are related as follows (see Proposition 4.6). Let

$$
A_{0}(M)_{\infty}:=\pi_{\infty}^{-1}\left(A_{0}(M)\right) \quad \text { and } \quad A_{0}(M)_{N}:=\operatorname{EXP} \circ l_{N, k_{c}}\left(A_{0}(M)_{\infty}\right),
$$

where

$$
\pi_{\infty}: \widetilde{\mathbb{C}}^{2} \rightarrow\left(\mathbb{C}^{*}\right)^{2}, \quad([\lambda ; p],[\lambda ; q]) \mapsto(\lambda, \mu)
$$

is the universal covering map, and for every integral class $k_{c} \in H^{1}(\partial V ; \mathbb{Z})$, define $l_{N, k_{c}}: \widetilde{\mathbb{C}}^{2} \rightarrow \mathbb{C}^{2}$ by

$$
\begin{aligned}
l_{N, k_{c}}([\lambda ; p],[\mu ; q]):=\left(\frac{1}{N}\left(\log (\lambda)+2 \pi i p-\pi i(N+1) k_{c}(l)\right)\right. & \\
& \left.\frac{1}{N}\left(\log (\mu)+2 \pi i q-\pi i(N+1) k_{c}(m)\right)\right) .
\end{aligned}
$$

The restricted map $\pi_{\infty}: A_{0}(M)_{\infty} \rightarrow A_{0}(M)$ and the map $\pi_{N}: A_{0}(M)_{N} \rightarrow A_{0}(M)$, $(u, v) \mapsto\left(u^{N}, v^{N}\right)$, define respectively a $\mathbb{Z} \times \mathbb{Z}$ - and a $\mathbb{Z} / N \times \mathbb{Z} / N$-covering of $A_{0}(M)$.

Definition 4.12 The classical and quantum log holonomies are the maps on $Z_{\infty, 0}$ defined by the components of the classes $L([w ; f]) \in H^{1}(\partial V ; \mathbb{C}) \quad$ and $\quad \exp \circ L_{N, c}([w ; f]) \in H^{1}\left(\partial V ; \mathbb{C}^{*}\right), \quad[w ; f] \in Z_{\infty, 0}$, in the basis $(l, m)$ of $\pi_{1}(\partial V)$ :

$$
\begin{aligned}
\operatorname{loghol}_{\infty}: Z_{\infty, 0} & \rightarrow A_{0}(M)_{\infty}, \\
{[w ; f] } & \mapsto(L([w ; f])(l), L([w ; f])(m)), \\
\operatorname{loghol}_{N, c}: Z_{\infty, 0} & \rightarrow A_{0}(M)_{N}, \\
{[w ; f] } & \mapsto\left(e^{L_{N, c}([w ; f])(l)}, e^{L_{N, c}([w ; f])(m)}\right) .
\end{aligned}
$$


Note that (32) and (34) imply that $\operatorname{loghol}_{\infty}\left(\right.$ resp. $\left.\operatorname{loghol}_{N, c}\right)$ maps into $A_{0}(M)_{\infty}$ (resp. $\left.A_{0}(M)_{N}\right)$. The identification (12) of $\mathcal{W}_{\infty}^{s}$ as a product of Riemann surfaces, and the fact that $Z_{\infty} \subset G_{0}(T, \tilde{b})_{\infty}$ is an analytic subspace imply that both $\operatorname{loghol}_{\infty}$ and $\operatorname{loghol}_{N, c}$ are analytic maps.

Remark 4.13 If one removes the assumption (31), the maps $\operatorname{loghol}_{\infty}$ and $\operatorname{loghol}_{N, c}$ extend to the whole of $Z_{\infty}$. Then $\operatorname{loghol}_{\infty}$ maps $Z_{\infty}$ to the union of four connected components $A_{0}(M)_{\infty}^{\epsilon, \epsilon^{\prime}}:=\left(\pi_{\infty}^{\epsilon, \epsilon^{\prime}}\right)^{-1}\left(A_{0}(M)\right)$, where $\pi_{\infty}^{\epsilon, \epsilon^{\prime}}: \widetilde{\mathbb{C}}^{2} \rightarrow\left(\mathbb{C}^{*}\right)^{2}$, $([\lambda ; p],[\mu ; q]) \mapsto\left(\epsilon \lambda, \epsilon^{\prime} \mu\right), \epsilon, \epsilon^{\prime} \in\{-1,+1\}$. The target of $\operatorname{loghol}_{N, c}$ becomes the union of the spaces EXP $\circ l_{N, k_{c}}\left(A_{0}(M)_{\infty}^{\epsilon, \epsilon^{\prime}}\right)$. The component $A_{0}(M)_{\infty}=A_{0}(M)_{\infty}^{0,0}$ is a natural one: we explain in Section 4D its relation with the Chern-Simons line bundle over $\partial V$.

We deduce a lemma immediately from the formulas (26) and (37).

Lemma 4.14 For every $N$ and every global charge $c$ on $(T, \tilde{b})$ we have a commutative diagram:

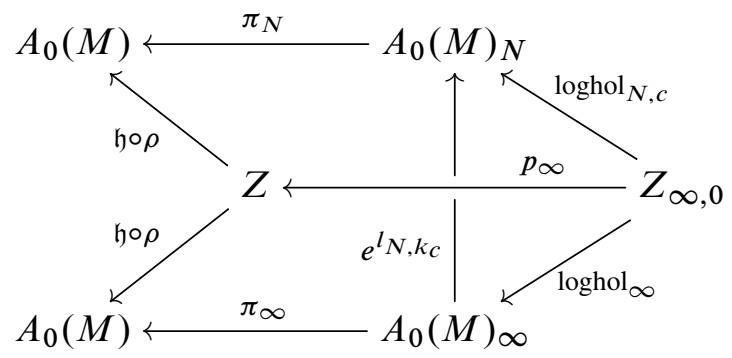

Here $l_{N, k_{c}}$ is defined by (37), using the charge boundary weight $k_{c}$, and we denote $\operatorname{EXP} \circ l_{N, k_{c}}$ by $e^{l_{N, k_{c}}}$.

Let $\mathcal{F}$ be any finite family of rich components, possibly contained in different gluing varieties associated to different weakly branched triangulations of $\widehat{V}$. Recall Proposition 4.6. Define the following nonempty Zariski open subsets of $X_{0}(M)$ :

$$
\Omega_{\mathcal{F}}=\bigcap_{Z \in \mathcal{F}} \rho\left(\Omega_{Z}\right), \quad \Omega_{\mathcal{F}}^{0}=\bigcap_{Z \in \mathcal{F}} \rho\left(\Omega_{Z}^{0}\right) .
$$

If $Z \in \mathcal{F}$ is a rich component of $G(T, \tilde{b})$, define

$$
\Omega_{\mathcal{F}}(Z):=\rho_{\mid Z}^{-1}\left(\Omega_{\mathcal{F}}\right), \quad \Omega_{\mathcal{F}}^{0}(Z):=\rho_{\mid Z}^{-1}\left(\Omega_{\mathcal{F}}^{0}\right)
$$

and

$$
\Omega_{\mathcal{F}}(Z)_{\infty, 0}:=p_{\infty}^{-1}\left(\Omega_{\mathcal{F}}(Z)\right) \cap Z_{\infty, 0}, \quad \Omega_{\mathcal{F}}(Z)_{N}:=\operatorname{EXP} \circ l_{N, *_{b}, c}\left(\Omega_{\mathcal{F}}(Z)_{\infty, 0}\right) .
$$


We have:

- $\Omega_{\mathcal{F}}^{0} \subset \Omega_{\mathcal{F}}$.

- The restriction of $\rho$ to $\Omega_{\mathcal{F}}(Z)$ is a rational regular map which is one-to-one onto $\Omega_{\mathcal{F}}$. Moreover, the nonnegative point $w_{h}^{Z} \in Z$ such that $\rho\left(w_{h}^{Z}\right)=\rho_{\text {hyp }}$ belongs to $\Omega_{\mathcal{F}}(Z)$.

- For every component $Z \in \mathcal{F}$, the restriction of $\rho$ to $\Omega_{\mathcal{F}}^{0}(Z)$ is a rational regular isomorphism onto $\Omega_{\mathcal{F}}^{0}$.

The following lemma is an immediate consequence of Proposition 4.9.

Lemma 4.15 Let $\mathcal{F}$ be any finite family of rich components of gluing varieties associated to weakly branched ideal triangulations of $\widehat{V}$. For every component $Z$ in $\mathcal{F}$, every point $\rho \in \Omega_{\mathcal{F}}$, and every $f$-weight $\left(k_{f}, h_{f}\right)$ of $(V, \rho)$ such that $\iota^{*}\left(h_{f}\right)=0$, there is a point $[w ; f]_{Z} \in \Omega_{\mathcal{F}}(Z)_{\infty, 0}$ such that $\left(L\left([w ; f]_{Z}\right), \gamma_{2}^{\prime}(f)\right)=\left(k_{f}, h_{f}\right)$.

Define

$$
A_{0, \mathcal{F}}(M):=\mathfrak{h}\left(\Omega_{\mathcal{F}}\right)
$$

and

$$
A_{0, \mathcal{F}}(M)_{\infty}:=\pi_{\infty}^{-1}\left(A_{0, \mathcal{F}}(M)\right), \quad A_{0, \mathcal{F}}(M)_{N}:=\operatorname{EXP} \circ l_{N, k_{c}}\left(A_{0, \mathcal{F}}(M)_{\infty}\right) .
$$

Replacing $G(T, \tilde{b})$ with $\Omega_{\mathcal{F}}(Z) \subset Z \subset G(T, \tilde{b})$, and $G_{0}(T, \tilde{b})_{\infty}$ with $\Omega_{\mathcal{F}}(Z)_{\infty, 0} \subset$ $Z_{\infty, 0} \subset G_{0}(T, \tilde{b})_{\infty}$, and taking the restrictions of each of the spaces and maps that form the sequence of analytic configurations $\left\{\mathcal{A}_{N}(T, \tilde{b}, c)\right\}$, one obtains a sequence of subconfigurations

$$
\mathcal{A}_{N}(c, Z, \mathcal{F}):=\left\{\Omega_{\mathcal{F}}(Z)_{\infty, 0}, \Omega_{\mathcal{F}}(Z)_{N}, p_{\infty}, p_{N}, l_{N, *_{b}, c}, \mathcal{H}_{1}, \mathcal{H}_{N}\right\} .
$$

By Lemma 4.14 it fits into a commutative diagram:

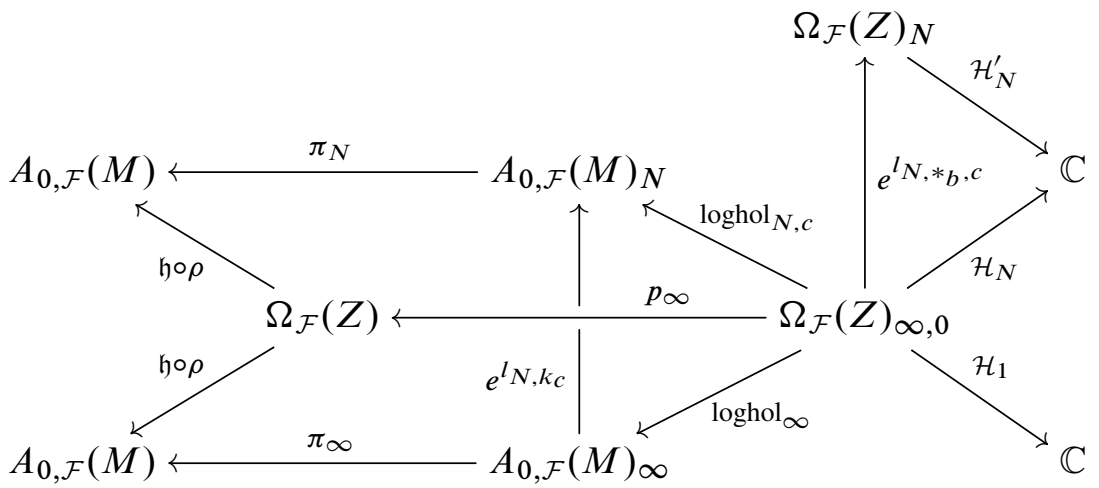


The following two results show that if we fix the bulk weight $h_{f}$ together with the $c$-weight $\left(h_{c}, k_{c}\right)$, then $\mathcal{H}_{1}$ and $\mathcal{H}_{N}$ factor through $\operatorname{loghol}_{\infty}$ and $\operatorname{loghol}_{N, c}$, and hence induce maps on $A_{0, \mathcal{F}}(M)_{\infty}$ and $A_{0, \mathcal{F}}(M)_{N}$, respectively.

Theorem 4.16 Let $\mathcal{F}$ be any finite family of rich components $Z$ of gluing varieties $G\left(T_{Z}, \tilde{b}_{Z}\right)$ associated to weakly branched ideal triangulations $\left(T_{Z}, \widetilde{b}_{Z}\right)$ of $\hat{V}$. Let $\rho \in \Omega_{\mathcal{F}}$, the $f$-weight $\left(h_{f}, k_{f}\right)$ and $[w ; f]_{Z} \in \Omega_{\mathcal{F}}(Z)_{\infty, 0}$ be as in Lemma 4.15, and $\left(h_{c}, k_{c}\right)$ be any $c$-weight realized on the triangulation $T_{Z}$ by a global charge $c_{Z}$. Then:

(1) For every odd $N \geq 3$ the scalar $\mathcal{H}_{N}^{\mathcal{F}}(\mathcal{P}):=\mathcal{H}_{N}\left(T_{Z}, \tilde{b}_{Z}, c_{Z}\right)\left([w ; f]_{Z}\right)$ is an invariant of the pattern $\mathcal{P}=\left(V, \rho, h_{f}, k_{f}, h_{c}, k_{c}\right)$ up to multiplication by $2 N^{\text {th }}$ roots of unity, and depends on $k_{f} \bmod \pi i N$ only.

(2) The scalar $\mathcal{H}_{1}^{\mathcal{F}}(\mathcal{P}):=\mathcal{H}_{1}\left(T_{Z}, \tilde{b}_{Z}\right)\left([w ; f]_{Z}\right)$ is an invariant of $\mathcal{P}=\left(V, \rho, h_{f}, k_{f}\right)$ up to multiplication by $6^{\text {th }}$ roots of unity, and is defined exactly if $\tilde{b}_{Z}$ is a branching.

Proof We have to show that, up to the phase ambiguities, $\mathcal{H}_{N}^{\mathcal{F}}(\mathcal{P})$ and $\mathcal{H}_{1}^{\mathcal{F}}(\mathcal{P})$ do not depend on the encoding of $\mathcal{P}$, ie the choice of $\left(T_{Z}, \widetilde{b}_{Z}, c_{Z}\right), Z$ and $[w ; f]_{Z}$. For that we use the $Q H$ transits of $[3 ; 4 ; 5]$, that we adapt to weakly branched triangulations in Section 7, in the case $N \geq 3$; for $N=1$ this adaptation follows easily from [4, Sections 4.2-4.3] and the argument of Proposition 8.2. As a result, given two realizations of the pattern $\mathcal{P}$, say

- $[w ; f]_{Z} \in \Omega_{\mathcal{F}}(Z)_{\infty, 0}$ included in some configuration $\mathcal{A}_{N}\left(c_{Z}, Z, \mathcal{F}\right)$,

- $\left[w^{\prime} ; f^{\prime}\right]_{Z^{\prime}} \in \Omega_{\mathcal{F}}\left(Z^{\prime}\right)_{\infty, 0}$ included in another configuration $\mathcal{A}_{N}\left(c_{Z^{\prime}}, Z^{\prime}, \mathcal{F}\right)$,

there exists a finite sequence of $\mathrm{QH}$ transits relating $[w ; f]_{Z}$ to $\left[w^{\prime \prime} ; f^{\prime \prime}\right]_{Z^{\prime}} \in \Omega_{\mathcal{F}}\left(Z^{\prime}\right)_{\infty, 0}$ included in a configuration $\mathcal{A}_{N}\left(c_{Z^{\prime}}^{\prime}, Z^{\prime}, \mathcal{F}\right)$ differing from $\mathcal{A}_{N}\left(c_{Z^{\prime}}, Z^{\prime}, \mathcal{F}\right)$ at most by the global charge, and such that

$$
\mathcal{H}_{N}\left(T_{Z}, \tilde{b}_{Z}, c_{Z}\right)\left([w ; f]_{Z}\right) \equiv_{2 N} \mathcal{H}_{N}\left(T_{Z^{\prime}}, \tilde{b}_{Z^{\prime}}, c_{Z^{\prime}}^{\prime}\right)\left(\left[w^{\prime \prime} ; f^{\prime \prime}\right]_{Z^{\prime}}\right) .
$$

It follows from the definition of $\Omega_{\mathcal{F}} \subset X_{0}(M)$ that $w^{\prime \prime}=w^{\prime}$. A further transit argument for flattenings and charges finally shows that

$$
\mathcal{H}_{N}\left(T_{Z^{\prime}}, \tilde{b}_{Z^{\prime}}, c_{Z^{\prime}}^{\prime}\right)\left(\left[w^{\prime} ; f^{\prime \prime}\right]_{Z^{\prime}}\right) \equiv_{2 N} \mathcal{H}_{N}\left(T_{Z^{\prime}}, \tilde{b}_{Z^{\prime}}, c_{Z^{\prime}}\right)\left(\left[w^{\prime} ; f^{\prime}\right]_{Z^{\prime}}\right) .
$$

Since the QH state sums (21) depend only on the reduction mod $N$ of flattenings, the dependence of $\mathcal{H}_{N}^{\mathcal{F}}, N \geq 3$, on $k_{f} \bmod \pi i N$ follows immediately. 
The above constructions depend on the choice of $\mathcal{F}$. A canonical choice is the family $\mathcal{F}_{\mathrm{EP}}$ of rich components $Z$ contained in any weakly branched EP-triangulation of $\hat{V}$ (see Proposition 2.2). By definition, the analytic configuration $\mathcal{A}_{N}(Y)$ of $Y=\left(V,\left(h_{c}, k_{c}\right)\right)$ is the family of analytic subconfigurations $\mathcal{A}_{N}\left(c, Z, \mathcal{F}_{\mathrm{EP}}\right)$ for varying charges and rich components $Z \in \mathcal{F}_{\mathrm{EP}}$.

Corollary 4.17 The invariants $\mathcal{H}_{1}^{\mathcal{F}_{\mathrm{EP}}}(\mathcal{P})$ and $\mathcal{H}_{N}^{\mathcal{F}_{\mathrm{EP}}}(\mathcal{P})$ of patterns $\mathcal{P}$ over $M$, such that $\mathcal{P}=\left(V, \rho, h_{f}, k_{f}, h_{c}, k_{c}\right)$ with $\rho \in \Omega_{\mathcal{F}_{\mathrm{EP}}}$, fixed $c$-weight $\left(h_{c}, k_{c}\right)$, and fixed bulk $f$-weight $h_{f} \in H^{1}(V ; \mathbb{Z} / 2 \mathbb{Z})$ such that $\iota^{*}\left(h_{f}\right)=0$, define respectively an analytic function

$$
\mathcal{H}_{1}^{\mathcal{F}_{\mathrm{EP}}, h_{f}}: A_{0, \mathcal{F}_{\mathrm{EP}}}(M)_{\infty} \rightarrow \mathbb{C}
$$

and a regular rational function

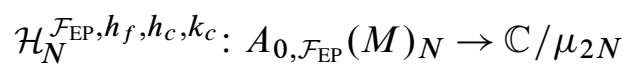

that give rise to a commutative diagram:

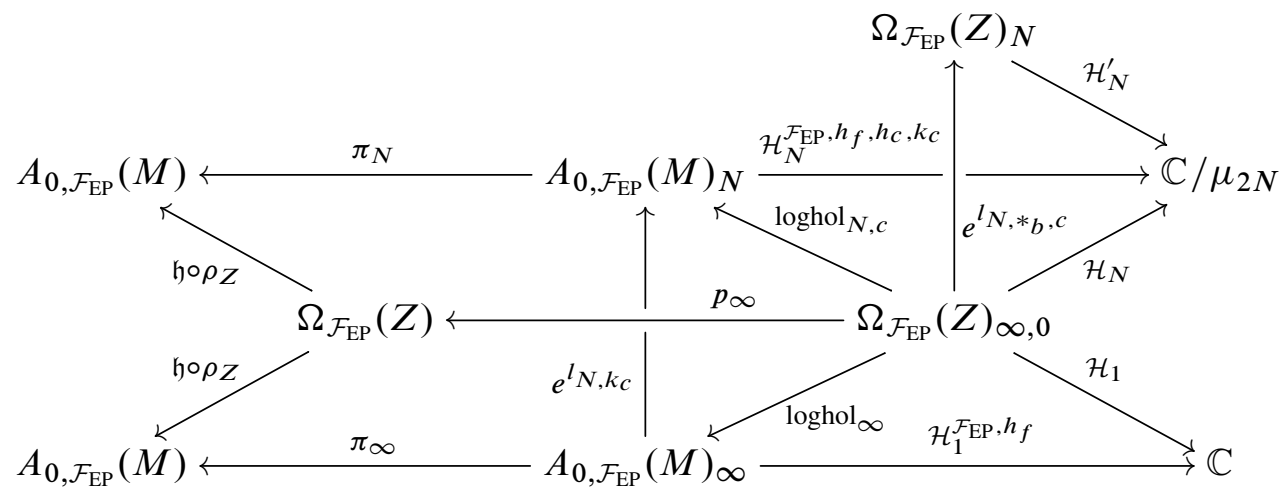

Proof Any point $x$ in the image of $\operatorname{loghol}_{\infty}$ or $\operatorname{loghol}_{N, c}$ determines a unique point $\rho=\mathfrak{h}^{-1}\left(\pi_{\infty}(x)\right)$ or $\mathfrak{h}^{-1}\left(\pi_{N}(x)\right)$ in $\Omega_{\mathcal{F}_{\mathrm{EP}}}$. If $x \in \operatorname{Im}(\operatorname{loghol} \infty)$, by Definition 4.10 it determines also a boundary $f$-weight $k_{f}$, and by Lemma 4.15 any boundary $f-$ weight $k_{f}$ for $\rho$ such that $\left(k_{f}-d_{\rho}\right) / i \pi$ is the zero class in $H^{1}(\partial V ; \mathbb{Z} / 2 \mathbb{Z})$ is realized by a point of $\pi_{\infty}^{-1}(\mathfrak{h}(\rho))$ in the image of $\operatorname{loghol}_{\infty}$ (see (28)). Hence the tuples $\left(x, h_{f}\right)$ and $\left(\rho, h_{f}, k_{f}\right)$ with $\iota^{*}\left(h_{f}\right)=0$ are in one-to-one correspondence. If $x \in \operatorname{Im}\left(\operatorname{loghol}_{N, c}\right)$ and a boundary $c$-weight $k_{c}$ is given, $x$ determines the reduction $\bmod \pi i N$ of $k_{f}$. Hence the tuples $\left(x, h_{f}, h_{c}, k_{c}\right)$ and $\left(\rho, h_{f}, k_{f} \bmod \pi i N, h_{c}, k_{c}\right)$ with $\iota^{*}\left(h_{f}\right)=0$ are in one-to-one correspondence. From this and Theorem 4.16, it follows that $\mathcal{H}_{1}$ and $\mathcal{H}_{N}$ descend to maps $\mathcal{H}_{1}^{\mathcal{F}_{\mathrm{EP}}, h_{f}}$ and $\mathcal{H}_{N}^{\mathcal{F}_{\mathrm{EP}}, h_{f}, h_{c}, k_{c}}$ defined on the image of loghol $\infty$ and $\operatorname{loghol}_{N, c}$ respectively. Since $\mathcal{H}_{1}$ is analytic and $\pi_{\infty}$ and $p_{\infty}$ are covering maps, $\mathcal{H}_{1}^{\mathcal{F}_{\mathrm{EP}}, h_{f}}$ is analytic too and it extends uniquely to an analytic function on $A_{0, \mathcal{F}_{\mathrm{EP}}}(M)_{\infty}$. 
Similarly $\mathcal{H}_{N}^{\mathcal{F}_{\mathrm{EP}}, h_{f}, h_{c}, k_{c}}$ can be extended in an unique way to a regular rational function on $A_{0, \mathcal{F}_{\mathrm{EP}}}(M)_{N}$.

Remark 4.18 The Epstein-Penner family $\mathcal{F}_{\mathrm{EP}}$ of rich components is only one of the possible choices. For instance, for every $M$ and $m \geq 2$ we can consider the family $\mathcal{F}_{m}$ of rich components of gluing varieties of weakly branched triangulations of $\hat{V}$ with no more than $m$ tetrahedra, or the family $\mathcal{F}_{m_{0}}$, where $m_{0}$ is the minimum $m$ such that $\mathcal{F}_{m} \neq \varnothing$. For example if $M$ is the complement of the knot $5_{2}$ in $S^{3}$, then $m_{0}=3$, while the Epstein-Penner subdivision is a triangulation made by 4 hyperbolic ideal tetrahedra. In practice, any rich component is suited to study the asymptotic behavior of the QHI at small deformations of the point $z_{\text {hyp }}$ representing the complete hyperbolic structure.

Proof of Theorem 1.1(1)-(2) For the first two statements, apply Theorem 4.16 to the family $\mathcal{F}=\mathcal{F}_{\mathrm{EP}}$, set $\Omega(M):=\Omega_{\mathcal{F}_{\mathrm{EP}}}$, and use Corollary 4.17 to pull back the covering $\pi_{N}$ via $\mathfrak{h}$. As explained in Remark 4.13, the assumption $\iota^{*}\left(h_{f}\right)=0$ is not necessary; in general we get functions $A_{0, \mathcal{F}_{\mathrm{EP}}}(M)_{N}^{\epsilon, \epsilon^{\prime}} \rightarrow \mathbb{C} / \mu_{2 N}$, where $\epsilon, \epsilon^{\prime} \in\{ \pm 1\}$.

Proof of Proposition 4.8(2) and 4.9(2) Let $(T, \tilde{b})$ be a weakly branched ideal triangulation of a pattern $(V, \rho,(h, k))$ over $M$. Denote by $\left(\Delta_{j}, b_{j}\right), j \in\{1, \ldots, s\}$, the 3 -simplices of $(T, \tilde{b})$. We need to review the Neumann-Zagier theory (see $[31 ; 32]$ ), that we will apply to the weakly branched triangulation $(T, \widetilde{b})$. Let

- $C_{1}\left(\Delta_{j}\right)$ be the $\mathbb{Z}$-module freely generated by the edges $e_{k}^{j}$ and $\left(e_{k}^{\prime}\right)^{j}, k \in$ $\mathbb{Z} / 3 \mathbb{Z}$, of $\Delta_{j}$

- $\quad \bar{J}_{j}$ be the quotient module of $C_{1}\left(\Delta_{j}\right)$ by the relations $e_{k}^{j}=\left(e_{k}^{\prime}\right)^{j}, k \in \mathbb{Z} / 3 \mathbb{Z}$;

- $J_{j}$ be the quotient module of $\bar{J}_{j}$ by the relation $e_{0}^{j}+e_{1}^{j}+e_{2}^{j}=0$.

Let $C_{1}$ be the $\mathbb{Z}$-module freely generated by the edges of $T$, and define $E(T):=$ $\bigoplus_{j} C_{1}\left(\Delta_{j}\right)$ (the $\mathbb{Z}$-module of abstract edges of $T$ ), $\bar{J}:=\bigoplus_{j} \bar{J}_{j}$, and $J:=\bigoplus_{j} J_{j}$. Consider on theses spaces the following 2-forms: the standard inner product $($, on $C_{1}, E(T)$ and $\bar{J}$, each defined with respect to the natural basis given by (cosets of) edges, and the signed antisymmetric bilinear form $\langle$,$\rangle on J$ given by

$$
\langle,\rangle=\bigoplus_{j} *_{j}\langle,\rangle_{j},
$$

where $\langle,\rangle_{j}$ is the standard antisymmetric bilinear form on $J_{j}$, defined in the basis $\left\{e_{0}^{j}, e_{1}^{j}\right\}$ by

$$
\left\langle e_{0}^{j}, e_{1}^{j}\right\rangle_{j}=1, \quad\left\langle e_{0}^{j}, e_{1}^{m}\right\rangle_{j}=0 \text { for } m \neq j
$$


Hence $\left\{e_{0}^{j}, e_{1}^{j}\right\}$ is a symplectic basis of $\langle$,$\rangle restricted to J_{j}$ when $\Delta_{j}$ has positive branching orientation $*_{j}=+1$, and is an antisymplectic basis of $J_{j}$ otherwise. Equivalently, $\left\{e_{0}^{j}, e_{1}^{j}\right\}$ is symplectic if the edges $e_{0}^{j},\left(e_{1}^{\prime}\right)^{j}, e_{2}^{j}$ have positive cyclic ordering as viewed from their common endpoint $v_{0}$ in $\Delta_{j}$ (in [31], the branchings are not needed and not used; one fixes positively cyclically ordered edges $e_{0}^{j},\left(e_{1}^{\prime}\right)^{j}, e_{2}^{j}$ independently for all $\Delta_{j}$, so that the signs $*_{j}$ occurring here are systematically replaced by +1 ). Define linear maps $\beta: C_{1} \rightarrow E(T)$ and $\beta^{*}: E(T) \rightarrow C_{1}$ by

$\beta(e)=\sum_{E \rightarrow e} E \quad$ and $\quad \beta^{*}\left(e_{k}^{j}\right)=*_{j}\left(p\left(e_{k+1}^{j}\right)-p\left(e_{k+2}^{j}\right)+p\left(\left(e_{k+1}^{\prime}\right)^{j}\right)-p\left(\left(e_{k+2}^{\prime}\right)^{j}\right)\right)$, and the same for $\beta^{*}\left(\left(e_{k}^{\prime}\right)^{j}\right)$, where indices are regarded mod 3 and $p: E(T) \rightarrow C_{1}$ is the identification map assigning to an abstract edge its coset in $T$. They induce maps (we keep the same notations)

$$
\beta: C_{1} \rightarrow J, \quad \beta^{*}: J \rightarrow C_{1} .
$$

Clearly, for any edge $e$ of $T$ we have

$$
\left(\beta^{*}\left(e_{k}^{j}\right), e\right)=\left\langle e_{k}^{j}, \beta(e)\right\rangle .
$$

Also, $\beta^{*}$ splits as $\beta^{*}: J \stackrel{\beta_{1}}{\longrightarrow} \bar{J} \stackrel{\beta_{2}}{\longrightarrow} C_{1}$, where

$$
\beta_{1}\left(e_{k}^{j}\right):=*_{j}\left(e_{k+1}^{j}-e_{k+2}^{j}\right) \quad \text { and } \quad \beta_{2}\left(e_{k}^{j}\right):=p\left(e_{k}^{j}\right)+p\left(\left(e_{k}^{\prime}\right)^{j}\right) .
$$

Similar to (40), $\beta_{1}$ and the natural projection $q: \bar{J} \rightarrow J$ are adjoint maps: for all $x \in \bar{J}$ and $a \in J$ we have

$$
\left(\beta_{1}(a), x\right)=\langle a, q(x)\rangle .
$$

Denote by $C_{0}$ the $\mathbb{Z}$-module freely generated by the vertices of $T$, and by $\alpha^{*}: C_{1} \rightarrow C_{0}$ the map which assigns to an edge the sum of its vertices. One has $\operatorname{Im}\left(\beta^{*}\right) \subset \operatorname{Ker}\left(\alpha^{*}\right)$, and interpreting the elements of $\operatorname{Ker}\left(\alpha^{*}\right)$ as unoriented $1-$ cycles in $T$, there is an isomorphism

$$
\operatorname{Ker}\left(\alpha^{*}\right) / \operatorname{Im}\left(\beta^{*}\right) \cong H_{1}(T ; \mathbb{Z} / 2 \mathbb{Z}) .
$$

It is not hard to see that $\operatorname{Im}(\beta) \subset \operatorname{Ker}\left(\beta^{*}\right)$. Put $H:=\operatorname{Ker}\left(\beta^{*}\right) / \operatorname{Im}(\beta)$. The 2 -form $\langle$,$\rangle on J$ descends to $H$. Since $\operatorname{Ker}\left(\beta^{*}\right)=\operatorname{Im}(\beta)^{\perp},\langle$,$\rangle is nondegenerate on$ $H / \operatorname{Tors}(H)$. In fact, it is shown in [31] that there is an exact sequence

$$
0 \rightarrow H \stackrel{\left(\gamma^{\prime}, \gamma_{2}^{\prime}\right)}{\longrightarrow} H^{1}(\partial V ; \mathbb{Z}) \oplus H^{1}(V ; \mathbb{Z} / 2 \mathbb{Z}) \stackrel{r-l^{*}}{\longrightarrow} H^{1}(\partial V ; \mathbb{Z} / 2 \mathbb{Z}) \rightarrow 0 .
$$


Moreover, denoting by $\cdot$ the intersection product on $H_{1}(\partial V ; \mathbb{Z})$, and by $\gamma=\mathrm{PD} \circ \gamma^{\prime}$ the map $\gamma^{\prime}$ followed by the Poincaré duality isomorphism, for all $a, b \in H$ we have

$$
\gamma(a) \cdot \gamma(b)=2\langle a, b\rangle .
$$

The map $\gamma$ is defined as follows. Recall the notations introduced before Definition 4.10. For any 2 -face $F$ of $\partial T_{0}$ which is a boundary section of the truncated tetrahedron of $T_{0}$ corresponding to $\Delta_{j}$, let us write $F \rightarrow \Delta_{j}$, and denote by $s_{k}^{j, F}$ the edge of $F$ which is opposite to the vertex of $F$ that belongs to the edge $e_{k}^{j}$ or $\left(e_{k}^{\prime}\right)^{j}$, with the positive orientation as viewed from that vertex. Then the linear map

$$
\bar{\gamma}: \bar{J} \rightarrow C_{1}\left(\partial T_{0}\right), \quad e_{k}^{j} \mapsto \sum_{F \rightarrow \Delta_{j}} s_{k}^{j, F}
$$

descends to

$$
\gamma: H \rightarrow H_{1}(\partial V ; \mathbb{Z})
$$

Conversely, let $q: \bar{J} \rightarrow J$ be the natural projection. Denote by $\partial T_{0}^{\prime}$ the cellulation of $\partial V$ dual to $\partial T_{0}$. Represent classes in $H_{1}(\partial V ; \mathbb{Z})$ by simplicial loops in $\partial T_{0}^{\prime}$. For all faces $F \rightarrow \Delta_{j}$, denote by $a_{k}^{j, F}$ the simplicial arc in $\partial T_{0}^{\prime} \cap F$ which faces the vertex of $F$ that belongs to the edge $e_{k}^{j}$ or $\left(e_{k}^{\prime}\right)^{j}$, with the positive orientation as viewed from that vertex. Define a linear map

$$
\bar{\delta}: C_{1}\left(\partial T_{0}^{\prime}\right) \rightarrow \bar{J}, \quad a_{k}^{j, F} \mapsto e_{k}^{j} .
$$

Then $q \bar{\delta}$ descends to a map $\delta: H_{1}(\partial V ; \mathbb{Z}) \rightarrow H$. Moreover, for all $x \in H_{1}(\partial V ; \mathbb{Z})$ we have

$$
\gamma \circ \delta(x)=2 x
$$

The identity (44) follows from (47), together with the simple fact that for any simplicial loop $C$ in $\partial T_{0}^{\prime}$, we have

$$
C \cdot \bar{\gamma}\left(e_{k}^{j}\right)=*_{j}\left\langle\bar{\delta}(C), e_{k}^{j}\right\rangle_{j}
$$

where the sign $*_{j}$ comes from the orientation induced by the basis $\left\{e_{0}^{j}, e_{1}^{j}\right\}$ of $J_{j}$, as discussed after (39). Hence $\gamma$ and $\delta$ are adjoint maps with respect to the form $\langle$, on $J$ and the intersection form on $H_{1}(\partial V ; \mathbb{Z})$ : for all $x \in H_{1}(\partial V ; \mathbb{Z})$ and $a \in H$,

$$
\gamma(a) \cdot x=\langle a, \delta(x)\rangle .
$$

Taking coefficients in $\mathbb{Z} / 2 \mathbb{Z}$ and simplicial loops in $T_{0}^{\prime}$ in normal position with respect to $T_{0}$, one defines similarly to $\delta$ a map $\delta_{2}: H_{1}(V ; \mathbb{Z} / 2 \mathbb{Z}) \rightarrow H \otimes(\mathbb{Z} / 2 \mathbb{Z})$. The map $\gamma_{2}^{\prime}$ 
in the above exact sequence is defined for all $a \in H \otimes(\mathbb{Z} / 2 \mathbb{Z})$ and $c \in H_{1}(V ; \mathbb{Z} / 2 \mathbb{Z})$ by

$$
\gamma_{2}^{\prime}(a)(c)=\left\langle a, \delta_{2}(c)\right\rangle
$$

With this material in hand, we can show that the maps $\gamma^{\prime}$ and $\gamma_{2}^{\prime}$ produce the boundary and the bulk weights of $V$. This is done for the charge weights in [31, Section 6], and for the flattening weights in [32, Section 9], with the further constraint that the weights are taken equal to 0 . For the sake of completeness, let us explain the case of the flattening weights; the adaptation to the charge weights is not difficult.

Consider the weakly branched ideal triangulation $(T, \tilde{b})$ of $(V, \rho,(h, k))$. By assumption, there is a point $w \in G(T, \tilde{b})$ with $\rho$ as holonomy. Take for each 3 -simplex $\left(\Delta_{j}, b_{j}\right)$ the (local) flattening of the form

$$
\left(\underline{l}_{0}^{j}, \underline{l}_{1}^{j}, \underline{l}_{2}^{j}\right):=\left(\log \left(w_{0}^{j}\right), \log \left(w_{1}^{j}\right), \log \left(w_{2}^{j}\right)+\epsilon i \pi\right), \quad \epsilon \in\{-1,+1\},
$$

and set $\underline{l}:=\left(\underline{l}_{0}^{j}, \underline{l}_{1}^{j}, \underline{l}_{2}^{j}\right)_{j}$. Consider the vector

$$
v_{\underline{l}}:=\sum_{j=1}^{s} \underline{l}_{1}^{j} e_{0}^{j}-\underline{l}_{0}^{j} e_{1}^{j} \in J \otimes \mathbb{C} .
$$

We have

$\beta_{1}\left(v_{\underline{l}}\right)=\sum_{j=1}^{s} *_{j}\left(\underline{l}_{0}^{j} e_{0}^{j}+\underline{l}_{1}^{j} e_{1}^{j}-\left(\underline{l}_{0}^{j}+\underline{l}_{1}^{j}\right) e_{2}^{j}\right)=\sum_{j=1}^{s} *_{j}\left(\underline{l}_{0}^{j} e_{0}^{j}+\underline{l}_{1}^{j} e_{1}^{j}+\underline{l}_{2}^{j} e_{2}^{j}\right) \in \bar{J} \otimes \mathbb{C}$.

So $\beta_{1}\left(v_{\underline{l}}\right)$ represents the map assigning the value $*_{j} \underline{l}_{k}^{j}$ to the abstract edges $e_{k}^{j}$ and $\left(e_{k}^{\prime}\right)^{j}$ of $T$. Recall that for every $\bar{a} \in \bar{J}, \beta_{2}(\bar{a})$ computes at every edge $e \in C_{1}$ the sum of the coefficients of $\bar{a}$ attached to the abstract edges having coset $e$. Then, $\beta^{*}=\beta_{2} \circ \beta_{1}$, the gluing Equation (8), and the argument of Lemma 2.7 imply that $\beta^{*}\left(v_{\underline{l}}\right) \in 2 \pi i C_{1}$ (note that in the lemma the label $\varnothing$ is attached to edges of the type $e_{2}^{j}$ and $\left.\left(e_{2}^{\prime}\right)^{j}\right)$. By (42) we deduce that $\beta^{*}\left(v_{\underline{l}}\right) / \pi i$ represents the 0 class in $H_{1}(T ; \mathbb{Z} / 2 \mathbb{Z})$, and so there exists $\underline{f} \in J$ such that $\beta^{*}(\underline{f})=\beta^{*}\left(v_{\underline{l}}\right) / \pi i$. Put $v_{l}:=v_{\underline{l}}-\pi i \underline{f}$, and write its coefficients as

$$
v_{l}=\sum_{j=1}^{s} l_{1}^{j} e_{0}^{j}-l_{0}^{j} e_{1}^{j}=\sum_{j=1}^{s}\left(\log \left(w_{1}^{j}\right)+\pi i f_{1}^{j}\right) e_{0}^{j}-\left(\log \left(w_{0}^{j}\right)+\pi i f_{0}^{j}\right) e_{1}^{j} .
$$

Since $\beta^{*}\left(v_{l}\right)=0$, the collection $l:=\left(l_{0}^{j}, l_{1}^{j}, l_{2}^{j}\right)_{j}$ satisfies the edge relations $L(e)=1$ (see (22)). The tetrahedral relations are satisfied because any element of $\operatorname{Im}\left(\beta_{1}\right)$ has coefficient sum equal to 0 in each module $\bar{J}_{j}$. So $l$ defines a flattening of $w$. Consider 
the class $\left[v_{l}\right] \in H \otimes \mathbb{C}$. One can see that $\gamma^{\prime}\left(\left[v_{l}\right]\right)=L([w ; f])$ as follows. Consider the map

$$
\bar{\gamma}^{\prime \prime}: \bar{J} \otimes \mathbb{C} \rightarrow C^{1}\left(\partial T_{0}^{\prime} ; \mathbb{C}\right), \quad x \mapsto(C \mapsto(x, \bar{\delta}(C))) .
$$

Denote by $A \in J \otimes \mathbb{C}$ a representative of $a \in H \otimes \mathbb{C}$. Then $\bar{\gamma}^{\prime \prime}$ induces a map

$$
\gamma^{\prime \prime}: H \otimes \mathbb{C} \rightarrow H^{1}(\partial V ; \mathbb{C}), \quad a \mapsto \bar{\gamma}^{\prime \prime}\left(\beta_{1}(A)\right) .
$$

Using successively (41) and (49), we get

$$
\gamma^{\prime \prime}(a)([C])=\left(\beta_{1}(A), \bar{\delta}(C)\right)=\langle a, q \bar{\delta}([C])\rangle=\gamma(a) \cdot[C] .
$$

Hence $\gamma^{\prime \prime}=\gamma^{\prime}$. Clearly, $\gamma^{\prime \prime}\left(\left[v_{l}\right]\right)=\bar{\gamma}^{\prime \prime}\left(\beta_{1}\left(v_{l}\right)\right)$ is given by (26). This proves $\gamma^{\prime}\left(\left[v_{l}\right]\right)=L([w ; f])$. The collection $f:=\left(f_{0}^{j}, f_{1}^{j}, f_{2}^{j}\right)_{j}$ satisfies also the edge relations $\bmod 2$, hence it defines a class $\gamma_{2}^{\prime}(f) \in H^{1}(V ; \mathbb{Z} / 2 \mathbb{Z})$ as explained after (26). By construction we have the compatibility relations (28), so the pair $\left(L([w ; f]), \gamma_{2}^{\prime}(f)\right)$ is a flattening weight of $(V, \rho)$. Finally, take any other flattening weight $\left(k_{f}^{\prime}, h_{f}^{\prime}\right)$ of $(V, \rho)$. Comparing (2) and (28) we see that $k_{f}^{\prime}-L([w ; f]) \in H^{1}(V ; \pi i \mathbb{Z})$ and $\left(k_{f}^{\prime}-L([w ; f])\right) / \pi i=\iota^{*}\left(h_{f}^{\prime}-\gamma_{2}^{\prime}(f)\right) \bmod 2$. By the exact sequence (43), there exists $a \in H$ such that $\gamma^{\prime}(a)=\left(k_{f}^{\prime}-L([w ; f])\right) / \pi i$ and $\gamma_{2}^{\prime}(a)=h_{f}^{\prime}-\gamma_{2}^{\prime}(f)$. Then the vector $\beta_{1}\left(v_{l}+\pi i a\right) \in \bar{J} \otimes \mathbb{C}$ represents a flattening of $w$ with weight $\left(h_{f}^{\prime}, k_{f}^{\prime}\right)$.

\section{D Relation with Chern-Simons theory}

Recall that $V$ is the compact 3-manifold obtained by completing the cusp of $M$ with a torus. Chern-Simons theory with gauge group $\operatorname{PSL}(2, \mathbb{C})$ associates to $\partial V$ the Chern-Simons bundle $\mathcal{L}_{\partial V} \rightarrow X(\partial V)$, which is a $\mathbb{C}^{*}$-bundle with canonical connection 1-form and canonical inner product, and to $V$ the parallel Chern-Simons section $s_{V}: X(V) \rightarrow \iota^{*} \mathcal{L}_{\partial V}$, where $\iota: X(V) \rightarrow X(\partial V)$ is the restriction map [19; 25].

We are going to show that when the bulk $f$-weight $h_{f}$ is 0 , the bottom row of the diagram of Corollary 4.17 encodes the restriction of the pair $\left(\iota^{*} \mathcal{L}_{\partial V}, s_{V}\right)$ to $\Omega_{\mathcal{F}_{\mathrm{EP}}} \subset$ $X_{0}(M)$, identified as a component of $X(V)$. For arbitrary $h_{f}$ one gets a twisted version of $\left(\iota^{*} \mathcal{L}_{\partial V}, s_{V}\right)$. The proof relies on a certain number of results that we need to recall. Denote by $\operatorname{CS}\left(M^{\prime}\right) \in \mathbb{R} / \mathbb{Z}$ the Chern-Simons invariant of the Levi-Civita connection of a closed Riemannian 3-manifold $M^{\prime}$, and by CS $(M)$ Meyerhoff's extension to cusped hyperbolic manifolds $M$. By definition, $\operatorname{CS}(M)$ is the limit of $\operatorname{CS}\left(M_{n}^{\prime}\right)$ for any sequence $\left(M_{n}^{\prime}\right)$ of closed hyperbolic Dehn fillings of $M$ converging to $M$ in Dehn surgery space. In [32, Corollary 14.6 and Theorem 14.7], Neumann proved the following, translated to the notations of the present paper. 
Theorem 4.19 Let $Y$ be $M$ or a closed hyperbolic Dehn filling $M^{\prime}$ of $M$. Denote by $\rho_{Y}$ the hyperbolic holonomy of $Y$, identified as a character of $V \subset M^{\prime}$ in the case $Y=M^{\prime}$. If $Y=M$, put $k_{Y}=0$, and if $Y=M^{\prime}$, let $k_{Y}$ be any boundary $f$-weight of $V$ relative to $\rho_{Y}$ such that $k_{Y}$ vanishes on the meridian of the added solid torus. Then

$$
\mathcal{H}_{1}\left(V, \rho_{Y},\left(0, k_{Y}\right)\right)=\exp \left(\frac{2}{\pi} \operatorname{Vol}(Y)+2 \pi i \operatorname{CS}(Y)\right) .
$$

The expression on the right-hand side is related to the Chern-Simons section $s_{V}$ as follows. To any compact closed oriented 3-manifold $Y$, PSL (2, $\mathbb{C})-$ Chern-Simons theory associates a function $s_{Y}: X^{\prime}(Y) \rightarrow \mathbb{C}^{*}$ defined on the variety $X^{\prime}(Y)$ of $\operatorname{PSL}(2, \mathbb{C})$-characters of $Y$. If $Y$ is hyperbolic with holonomy $\rho_{Y}$, a classical result of Yoshida [37] gives

$$
s_{Y}\left(\rho_{Y}\right)=\exp \left(\frac{2}{\pi} \operatorname{Vol}(Y)+2 \pi i \operatorname{CS}(Y)\right) .
$$

Assume that $Y=V \cup_{\phi}\left(D^{2} \times S^{1}\right)$ is a closed hyperbolic Dehn filling of $M$ whose holonomy $\rho_{Y}$ lies in a sufficiently small neighborhood $D$ of $\rho_{\text {hyp }}$ in $X(V)=X(M)$ (here we identify $\rho(Y)$ with an augmented character of $V$ ). Then Kirk and Klassen [25, pages 554-556] showed that

$$
s_{Y}\left(\rho_{Y}\right)=\left\langle s_{V}\left(\rho_{Y}\right), s_{D^{2} \times S^{1}}\left(\rho_{Y}\right)\right\rangle,
$$

where $\langle$,$\rangle is the canonical inner product of \iota^{*} \mathcal{L}_{\partial V}$, and on the right-hand side $\rho_{Y}$ denotes also the augmented character induced on the glued solid torus $D^{2} \times S^{1}$. Explicitly, if we fix the gauge on $\iota^{*} \mathcal{L}_{\partial V}$ by taking as coordinates on $D$ the ones corresponding under the map $\mathfrak{h}$ to the standard $\log$ arithms $\log (\lambda)$ and $\log (\mu)$, which are equal to 0 at $\rho_{\text {hyp }}$, we have

$$
s_{V}\left(\rho_{\text {hyp }}\right)=\exp \left(\frac{2}{\pi} \operatorname{Vol}(M)+2 \pi i \operatorname{CS}(M)\right) .
$$

Then, if the Dehn filling instruction $\phi$ maps the meridian $\partial\left(D^{2} \times *\right)$ to $l^{q} m^{p}$ and the longitude $* \times S^{1}$ to $l^{s} m^{r}$ (so $p s-q r=1$ ), the formula (53) splits as

$$
\begin{aligned}
s_{Y}\left(\rho_{Y}\right)=s_{V}\left(\rho_{\text {hyp }}\right) \exp \left(-\frac{1}{2 \pi i} \int_{\rho_{\text {hyp }}}^{\rho_{Y}}(\log (\lambda)\right. & d \log (\mu)-\log (\mu) d \log (\lambda))) \\
& \times \exp (-(s \log (\lambda)+r \log (\mu))) .
\end{aligned}
$$

The first exponential gives the variation of $s_{V}$ between $\rho_{\text {hyp }}$ and $\rho_{Y}$ ( $s_{V}$ being a parallel section of $\iota^{*} \mathcal{L}_{\partial V}$, this exponential is the holonomy of the connection 1 -form of $\iota^{*} \mathcal{L}_{\partial V}$ between the two points); the product with $s_{V}\left(\rho_{\text {hyp }}\right)$ is thus the value of $s_{V}\left(\rho_{Y}\right)$ at the chosen gauge. The second exponential is the value of $s_{D^{2} \times S^{1}}\left(\rho_{Y}\right)$ in the 
gauge corresponding to the Dehn filling: $\log \left(\operatorname{hol}_{\partial\left(D^{2} \times *\right)}\right)=q \log (\lambda)+p \log (\mu)=2 \pi i$, $\log \left(\operatorname{hol}_{* \times S^{1}}\right)=s \log (\lambda)+r \log (\mu)$. Its argument is $-($ length $+i$ rotation angle) of the geodesic core of the surgery torus. The arguments of both exponentials can be collected in a single integral by changing the coordinates $\log (\lambda), \log (\mu)$ on $D$ to $\log (\lambda)+2 \pi i r$, $\log (\mu)-2 \pi i s$, that is, by working on a different leaf of the Riemann surface of these maps. Then the gauge $\log \left(\operatorname{hol}_{\partial\left(D^{2} \times *\right)}\right)$ vanishes, like $k_{Y}$ in Theorem 4.19.

We will show that $\left(\iota^{*} \mathcal{L}_{\partial V}, s_{V}\right)_{\mid \Omega_{\mathcal{F}_{\mathrm{EP}}}}$ is determined by $\mathcal{H}_{1}^{\mathcal{F}_{\mathrm{EP}}, 0}: A_{0, \mathcal{F}_{\mathrm{EP}}}(M)_{\infty} \rightarrow \mathbb{C}$. Because of Theorem 4.19 and (54), it is enough to identify the variation of $\mathcal{H}_{1}^{\mathcal{F}_{\mathrm{EP}}, 0}$ with the one of $s_{V}$, given by (55). This is the content of the following result.

Proposition 4.20 The function $\mathcal{H}_{1}^{\mathcal{F}_{\mathrm{EP}}, 0}$ descends to a parallel section $\mathcal{S}^{0}$ of a flat trivial $\mathbb{C}^{*}$-bundle $\mathcal{L}(M) \rightarrow A_{0, \mathcal{F}_{\mathrm{EP}}}(M)$ with canonical connection 1 -form and inner product, such that $\mathfrak{h}^{*}\left(\mathcal{L}(M), \mathcal{S}^{0}\right)$ is isomorphic to $\left(\iota^{*} \mathcal{L}_{\partial V}, s_{V}\right)_{\mid \Omega_{\mathcal{F}_{\mathrm{EP}}}}$.

Proof We define $\mathcal{L}(M)$ from the variation of the invariant $\mathcal{H}_{1}^{\mathcal{F}_{\mathrm{EP}}}$ of Theorem 4.16, like the Chern-Simons line bundle is defined from the Chern-Simons action [19; 25]. Hence $\mathcal{L}(M)$ is the quotient of $A_{0, \mathcal{F}_{\mathrm{EP}}}(M)_{\infty} \times \mathbb{C}^{*}$ under the action of $\mathbb{Z}^{2}$ given by

$$
(a, b) \cdot([\lambda ; p],[\mu ; q], z):=\left([\lambda ; p+a],[\mu ; q+b], z e^{b \log (\lambda)-a \log (\mu)}\right)
$$

for all $a, b \in \mathbb{Z}$. We have a $\mathbb{C}^{*}$-bundle projection

$$
\pi_{\infty}^{\prime}: \mathcal{L}(M) \rightarrow A_{0, \mathcal{F}_{\mathrm{EP}}}(M), \quad[[\lambda ; p],[\mu ; q], z] \mapsto(\lambda, \mu) .
$$

Denote by $\mathcal{L}(M)^{-1}$ the inverse $\mathbb{C}^{*}$-bundle, which is the same as $\mathcal{L}_{\mathcal{F}_{\mathrm{EP}}}(-M)$, where $-M$ denotes $M$ with reversed orientation. An inner product is defined by the bundle map

$$
\begin{aligned}
\langle,\rangle: \mathcal{L}(M) \times \mathcal{L}(M)^{-1} & \rightarrow \mathbb{C}^{*}, \\
\left(\left([\lambda ; p],[\mu ; q], z_{1}\right),\left([\lambda ; p],[\mu ; q], z_{2}\right)\right) & \mapsto z_{1} z_{2},
\end{aligned}
$$

where $\mathbb{C}^{*}$ is regarded as the bundle over a point. Also, a flat analytic connection 1 -form is defined by the restriction to $A_{0, \mathcal{F}_{\mathrm{EP}}}(M)_{\infty}$ of the 1 -form on $\widetilde{\mathbb{C}} \times \widetilde{\mathbb{C}}$ given by (we put $\left.l_{a}(\lambda):=\log (\lambda)+2 \pi i a\right)$

$$
\eta:=-\frac{1}{2 \pi i}\left(l_{p}(\lambda) d l_{q}(\mu)-l_{q}(\mu) d l_{p}(\lambda)\right) .
$$

By Lemma 4.15, given any even class $\theta \in H^{1}(\partial V ; 2 \mathbb{Z})$ and any point $[w ; f]_{Z} \in$ $\Omega_{\mathcal{F}_{\mathrm{EP}}}(Z)_{\infty, 0}$, the flattening $f$ can be modified to a flattening $f^{\prime}$ such that $\left[w ; f^{\prime}\right]_{Z} \in$ $\Omega_{\mathcal{F}_{\mathrm{EP}}}(Z)_{\infty, 0}$ and the corresponding boundary weights satisfy

$$
L\left(\left[w ; f^{\prime}\right]_{Z}\right)-L\left([w ; f]_{Z}\right)=\pi i \theta
$$


As in the proof of Proposition 4.8(2) and 4.9(2), denote by $v_{l}$ (resp. $v_{l^{\prime}}$ ) the vector in $H \otimes \mathbb{C}$ associated to the classical log branch at $[w ; f]_{Z}\left(\right.$ resp. $\left.\left[w ; f^{\prime}\right]_{Z}\right)$, and similarly denote by $v_{d} \in H \otimes(\mathbb{C} / 2 \pi i \mathbb{Z})$ the vector associated to $\left(\log \left(w_{0}^{j}\right), \log \left(w_{1}^{j}\right), \log \left(w_{2}^{j}\right)\right)_{j}$. Recall the class $d_{w} \in H^{1}(\partial V ; \mathbb{C} / 2 \pi i \mathbb{Z})$ in (28). Then $d_{w}=\gamma^{\prime}\left(v_{d}\right)$ and

$$
\pi i \theta=\gamma^{\prime}\left(v_{l^{\prime}}-v_{l}\right)=\gamma^{\prime}\left(\pi i \sum_{j=1}^{s}\left(\left(f^{\prime}\right)_{1}^{j}-f_{1}^{j}\right) e_{0}^{j}-\left(\left(f^{\prime}\right)_{0}^{j}-f_{0}^{j}\right) e_{1}^{j}\right) .
$$

Hence, denoting $\mathcal{H}_{1}^{\mathcal{F}_{\mathrm{EP}}}:=\mathcal{H}_{1}^{\mathcal{F}_{\mathrm{EP}}}\left(T_{Z}, \tilde{b}_{Z}\right)$,

$$
\begin{aligned}
\mathcal{H}_{1}^{\mathcal{F}_{\mathrm{EP}}}\left(\left[w ; f^{\prime}\right]_{Z}\right) & \mathcal{H}_{1}^{\mathcal{F}_{\mathrm{EP}}}\left([w ; f]_{Z}\right)^{-1} \\
& =\exp \left(\sum_{j=1}^{s} *_{j}\left(\left(\left(f^{\prime}\right)_{1}^{j}-f_{1}^{j}\right) \log \left(w_{0}^{j}\right)-\left(\left(f^{\prime}\right)_{0}^{j}-f_{0}^{j}\right) \log \left(w_{1}^{j}\right)\right)\right) \\
& =\exp \left(-\frac{1}{\pi i}\left\langle v_{l^{\prime}}-v_{l}, v_{d}\right\rangle\right) \\
& =\exp \left(-\frac{1}{2 \pi i}\left\langle\left\langle\gamma^{\prime}\left(v_{l^{\prime}}-v_{l}\right), \gamma^{\prime}\left(v_{d}\right)\right\rangle\right)\right) \\
& =\exp \left(\frac{1}{2}\left(\theta(m) \log \left(\operatorname{hol}_{l}(\rho(w))\right)-\theta(l) \log \left(\operatorname{hol}_{m}(\rho(w))\right)\right)\right)
\end{aligned}
$$

where the map hol $C, C$ a curve in $\partial V$, is defined in (23), and $\langle\langle\rangle$,$\rangle denotes the cup$ product followed by the evaluation on the fundamental class. The first equality follows from (17) and (21), the second is by the definition (38) of the symplectic pairing on $H \otimes \mathbb{C}$, the third follows from (44) and Poincare duality, and the last one uses that the symplectic basis of $H_{1}(\partial V ; \mathbb{Z})$ is $(l, m)$. By setting $\theta(m):=2 b$ and $\theta(l):=2 a$, we see from (56) and (58) that the function $\mathcal{H}_{1}^{\mathcal{F}_{\mathrm{EP}}, h_{f}}$ (with arbitrary bulk weight $h_{f}$ for the moment) descends from $A_{0, \mathcal{F}_{\mathrm{EP}}}(M)_{\infty}$ to a section of $\mathcal{L}(M)$. Denote it

$$
\mathcal{S}^{h_{f}}: A_{0, \mathcal{F}_{\mathrm{EP}}}(M) \rightarrow \mathcal{L}(M) .
$$

We show that this section is parallel with respect to the connection $\eta$ by a computation similar to (58). As remarked in [31, Lemma 10.2], for any $\mathbb{Q}$-vector space $E$, the skew symmetric bilinear form $\langle$,$\rangle on J$ induces a symmetric bilinear map

$B:(H \otimes E) \otimes(H \otimes E) \rightarrow E \wedge E$,

$(a \otimes v) \otimes(b \otimes w) \mapsto\langle a, b\rangle v \wedge w$.

Consider the map $\cdot E$ on $H_{1}(\partial V ; E)=H_{1}(\partial V ; \mathbb{Z}) \otimes E$ induced by the intersection product on $H_{1}(\partial V ; \mathbb{Z})$ :

$$
\begin{aligned}
\cdot_{E}: H_{1}(\partial V ; E) \otimes H_{1}(\partial V ; E) & \rightarrow E \wedge E, \\
(x \otimes v) \otimes(y \otimes w) & \mapsto(x \cdot y) v \wedge w .
\end{aligned}
$$


Denote again by $\gamma$ the map $\gamma \otimes \mathrm{Id}: H \otimes E \rightarrow H_{1}(\partial V ; E)$. By (44) we have a commutative diagram:

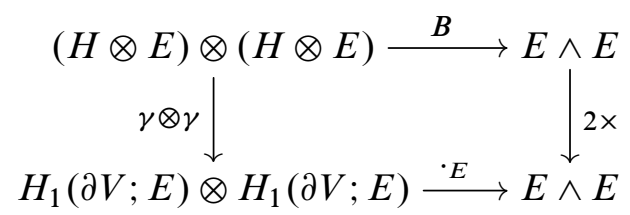

In particular, let $E=C^{\infty}\left(\mathcal{W}_{\infty}\right)$ and denote by $l_{0}^{j}, l_{1}^{j} \in E$ the classical log branch functions at the edges $e_{0}^{j}, e_{1}^{j}$ of $\Delta_{j}$. Put

$$
a=\sum_{j=1}^{s} e_{0}^{j} \otimes l_{1}^{j}-e_{1}^{j} \otimes l_{0}^{j} \in H \otimes E .
$$

For any point $[w ; f]_{Z} \in \Omega_{\mathcal{F}_{\mathrm{EP}}}(Z)_{\infty, 0}$ we have $\gamma(a)\left([w ; f]_{Z}\right)=l \otimes L\left([w ; f]_{Z}\right)(m)-$ $m \otimes L\left([w ; f]_{Z}\right)(l)$; dually the class $\gamma^{\prime}(a) \in H^{1}(\partial V ; E), \gamma^{\prime}(a)=\operatorname{PD} \circ \gamma(a)$, is given by $\gamma^{\prime}(a)\left([w ; f]_{Z}\right)=L\left([w ; f]_{Z}\right) \in H^{1}(\partial V ; \mathbb{C})$. Moreover, in $E \wedge E$ we have

$$
2 \sum_{j=1}^{s} *_{j}\left(l_{0}^{j} \wedge l_{1}^{j}\right)=B(a, a)=\frac{1}{2} \gamma(a) \cdot{ }_{E} \gamma(a)=L(\cdot)(l) \wedge L(\cdot)(m) .
$$

Applying the homomorphism $E \wedge E \rightarrow \Omega^{1}\left(\mathcal{W}_{\infty}\right), f \wedge g \mapsto f d g-g d f$, we deduce

$$
2 \sum_{j=1}^{s} *_{j}\left(l_{0}^{j} d l_{1}^{j}-l_{1}^{j} d l_{0}^{j}\right)=L(\cdot)(l) d L(\cdot)(m)-L(\cdot)(m) d L(\cdot)(l) .
$$

For any two points $[w ; f]_{Z},\left[w^{\prime} ; f^{\prime}\right]_{Z}$ connected by a continuous path $\left(\gamma^{1}, \ldots, \gamma^{s}\right)$ in $\Omega_{\mathcal{F}_{\mathrm{EP}}}(Z)_{\infty, 0}$ we thus get

$$
\mathcal{H}_{1}^{\mathcal{F}_{\mathrm{EP}}}\left(\left[w^{\prime} ; f^{\prime}\right]_{Z}\right) \mathcal{H}_{1}^{\mathcal{F}_{\mathrm{EP}}}\left([w ; f]_{Z}\right)^{-1}=\exp \left(2 \sum_{j=1}^{s} *_{j} \int_{\gamma^{j}} \eta\right)=\exp \left(\int_{\gamma} \eta\right)
$$

where $\gamma \subset A_{0, \mathcal{F}_{\mathrm{EP}}}(M)_{\infty}$ is the image of $\left(\gamma^{1}, \ldots, \gamma^{s}\right)$ under the map loghol ${ }_{\infty}$. Hence $\mathcal{S}^{h_{f}}$ is a parallel section of $\mathcal{L}(M)$. Multiplying coordinates by $4 \pi i$ identifies $\mathfrak{h}^{*} \mathcal{L}(M)$ with the restriction of $\iota^{*} \mathcal{L}_{\partial V}$ to $\Omega_{\mathcal{F}_{\mathrm{EP}}}$; see [25, end of Section 3 and pages 555-556]. Finally, as described before the claim, from (55) and (59) we get $\mathfrak{h}^{*} \mathcal{S}^{0}=\left(s_{V}\right) \mid \Omega_{\mathcal{F}_{\mathrm{EP}}}$.

Remark 4.21 (1) In [25] the Chern-Simons bundle is defined over the variety of characters $X^{\prime}(\partial V)$ instead of the variety of augmented characters $X(\partial V)$; the coordinates on $X^{\prime}(\partial V)$ are $1 / 2 \pi i$ times the logarithms of the eigenvalues at a pair of meridian and longitude curve on $\partial V$. A fundamental domain for the action of $\mathbb{Z} \times \mathbb{Z}$ 
on coordinates is $([0 ; 1 / 2] \times \mathbb{R}) \times([0 ; 1] \times \mathbb{R})$. The rescaling factor $4 \pi i$ at the end the proof replaces these coordinates by the logarithms of the squared eigenvalues, so that a fundamental domain for the action of $\mathbb{Z} \times \mathbb{Z}$ on $A_{0, \mathcal{F}_{\mathrm{EP}}}(M)_{\infty}$ is $([0 ; 2 i \pi] \times \mathbb{R})^{2}$.

(2) $\mathcal{L}(M)$ is the restriction to $A_{0, \mathcal{F}_{\mathrm{EP}}}(M)$ of a bundle $\mathcal{L}\left(T^{2}\right)$ over $X\left(T^{2}\right)=X(\partial V)$ defined by the action (56). Of course $\mathcal{L}\left(T^{2}\right)$ is isomorphic to $\mathcal{L}_{\partial V}$. Its connection 1-form (57) has curvature $F_{\eta}=(-1 /(i \pi)) d \log (\lambda) \wedge d \log (\mu)$, its Euler class is represented by $\left(-1 /\left(2(i \pi)^{2}\right)\right) d \log (\lambda) \wedge d \log (\mu)$, and its Euler number is -2 .

\section{Remarks 4.22 Problems and perspectives}

(a) The $\mathbb{Z}^{2}$-equivariant analytic function $\mathcal{H}_{1}^{\mathcal{F}_{\mathrm{EP}}, 0}$ defined on $A_{0, \mathcal{F}_{\mathrm{EP}}}(M)_{\infty}$ is the classical analog of the regular rational functions $\mathcal{H}_{N}^{\mathcal{F}} \mathcal{F}_{\mathrm{EP}}, 0, h_{c}, k_{c}$ on $A_{0, \mathcal{F}_{\mathrm{EP}}}(M)_{N}$. One expects that a refinement of the $\mathbb{Z}^{2}$-action appears in the quantum world, involving the quantum torus algebra generated by $\hat{l}, \hat{m}$ such that $\hat{l} \hat{m}=\zeta \hat{m} \hat{l}$, where $\zeta$ is a primitive $N^{\text {th }}$ root of 1 and $(l, m)$ a geometric basis of $H_{1}(\partial V ; \mathbb{Z})$ :

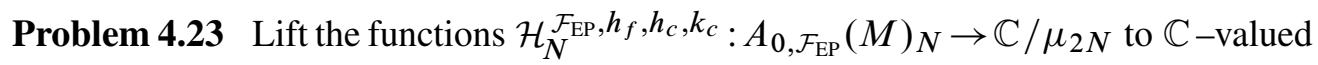
functions defined on some covering space of $A_{0, \mathcal{F}_{\mathrm{EP}}}(M)_{N}$.

(b) By Theorem 1.1(2), the functions $\mathcal{H}_{N}^{\mathcal{F}_{\mathrm{EP}}, h_{f}, h_{c}, k_{c}}$ yield $\mathbb{C} / \mu_{2 N}$-valued rational functions on a covering space $\tilde{X}_{0}(M)_{N}$ of $X_{0}(M)$. On the other hand, the field $\mathbb{C}\left(\tilde{X}_{0}(M)_{N}\right)$ is a finite extension of $\mathbb{C}\left(X_{0}(M)\right)$, and $\mathbb{C}\left(X_{0}(M)\right)$ is generated by the functions of augmented characters associated to hol $_{l}$ and hol $_{m}$ by pull-back via the map $\mathfrak{h}: X_{0}(M) \rightarrow A_{0}(M)$.

Problem 4.24 Describe $\mathcal{H}_{N}^{\mathcal{F}_{\mathrm{EP}}, h_{f}, h_{c}, k_{c}}$ in terms of functions of augmented characters, or as a meromorphic function on the smooth projective model of $\widetilde{X}_{0}(M)_{N}$.

For instance, a property that is obvious from the state sum formulas is that the poles of $\mathcal{H}_{N}^{\mathcal{F}_{\mathrm{EP}}, h_{f}, h_{c}, k_{c}}$ cover ideal points of $X_{0}(M)$.

By a theorem of Bullock [11], the ring of $\operatorname{SL}(2, \mathbb{C})$-characters $\mathbb{C}[X(M)]$ is isomorphic to $K_{-1}(M) / \sqrt{0}$, where $K_{-1}(M)$ is the Kauffman bracket skein module of $M$ specialized at $A=-1$, with its natural algebra structure, and $\sqrt{0}$ its nilradical.

Problem 4.25 Find skein-theoretic constructions of $\mathcal{H}_{N}^{\mathcal{F}} \mathcal{E P}_{\mathrm{EP}}, h_{f}, h_{c}, k_{c}$. 


\section{QHFT partition functions}

For simplicity we consider only the QHFT patterns with topological support ( $\left.W, L, h_{c}\right)$, where $W$ is a closed oriented connected 3-manifold. In that case only the bulk $c-$ weight $h_{c}$ occurs. In order to build the analytic configurations of $\left(W, L, h_{c}\right)$ we consider quasiregular triangulations $T$ of $W$ (see Proposition 2.3), and we require that $L$ is realized by a Hamiltonian subcomplex $H$ of the 1 -skeleton of $T$. Such a pair $(T, H)$ is called a distinguished triangulation of $(W, L)$.

Definition 5.1 A global charge $c$ on $(T, H)$ is a rough global charge on $T$ satisfying the following additional global constraint on the total edge charges: $C(e)=0$ if $e$ is an edge of $H$, and $C(e)=2$ otherwise.

Note that any global charge on $(T, H)$ encodes $H$, ie the link $L$. Let us turn to the combinatorial encoding of $\left(W, L, h_{c}\right)$. Similarly to Proposition 4.8 and 4.9 we arrive at the following proposition; see $[3 ; 4 ; 5]$.

Proposition 5.2 (1) Every pair $(W, L)$ has quasiregular distinguished triangulations $(T, H)$.

(2) Every global charge $c$ on $(T, H)$ determines a bulk $c$-weight $h_{c}$ of $W$.

(3) For every bulk $c$-weight $h_{c}$ of $W$ and every distinguished triangulation $(T, H)$ of $(W, L)$, there is a global charge $c$ on $(T, H)$ with bulk $c$-weight equal to $h_{c}$.

Let us fix a quasiregular distinguished triangulation $(T, H)$ of $(W, L)$, a global charge $c$ on $(T, H)$, and a branching $b$ on $T$ (it exists).

Proposition 5.3 (1) Every point $[w ; f] \in G_{0}(T, b)_{\infty}$ determines a character $\rho=$ $\rho(w)$ and a bulk $f$-weight $h_{f}$, so that $\left(W, L, \rho, h_{f}, h_{c}\right)$ is a pattern with topological support $\left(W, L, h_{c}\right)$.

(2) For every pattern $\left(W, L, \rho, h_{f}, h_{c}\right)$ there is a point $[w ; f] \in G_{0}(T, b)_{\infty}$ with holonomy $\rho$ and bulk $f$-weight $h_{f}$.

The construction of the $\mathrm{QHI}$ of $\left(W, L, h_{c}\right)$ is achieved by the following proposition, which is improved in Section 8 with respect to the QHI sign anomaly. The proof is similar to that of Theorem 4.16. For every odd $N \geq 3$ consider the analytic configurations $\mathcal{A}_{N}(T, b, c)$. The defining equations of the algebraic variety $G_{0}(T, b, c)_{N}$ are:

- $W^{\prime}(e)=\zeta^{-1}$ for every edge $e$ of $T$ not contained in $H$.

- $W^{\prime}(e)=1$ for every edge $e$ of $T$ contained in $H$. 
Proposition 5.4 For every pattern $\mathcal{P}=\left(W, L, \rho, h_{f}, h_{c}\right)$, every distinguished branched quasiregular triangulation $(T, b, H)$ of $(W, L)$, every global charge $c$ on $(T, H)$ with bulk $c$-weight equal to $h_{c}$, and every point $[w ; f] \in G_{0}(T, b)_{\infty}$ with holonomy $\rho$ and bulk $f$-weight $h_{f}$, the scalars

- $\mathcal{H}_{1}(\mathcal{P}):=\mathcal{H}_{1}(T, b)([w ; f])$

- $\mathcal{H}_{N}(\mathcal{P}): \equiv_{2 N} \mathcal{H}_{N}(T, b, c)([w ; f])$

do not depend on the choice of $(T, b, c)$ and $[w ; f]$, and hence define invariants of $\mathcal{P}$.

By definition, the analytic configuration $\mathcal{A}_{N}\left(W, L, h_{c}\right)$ is the family of analytic configurations $\mathcal{A}_{N}(T, b, c)$ over the triples $(T, b, c)$ as in Proposition 5.4.

Remark 5.5 The state sum normalization factor $a_{N}(T, \tilde{b})$ includes a term $N^{-v}$. Other choices are possible. In [6] we used instead $N^{2-v}$ in order to get Theorem 1.2.

For general QHFT patterns the constructions are more elaborated; in particular, one requires that $H$ is Hamiltonian in the 1-skeleton of $T$ from which the nonmanifold vertices have been removed, and that in the normalization factor $N^{-v}, v$ is the number of manifold (ie internal) vertices. Anyway, the results have the same flavor.

\section{$6 \mathcal{N}$-graph calculus}

As every QH state sum is the total contraction of a tensor network supported by a $\mathcal{N}$-graph, one needs to describe the main features of a calculus based on such diagrams.

\section{A $\mathcal{N}$-graphs representing the same weakly branched triangulation}

Using the $\mathcal{N}$-graph decoding of Section 2 it is easy to see that two $\mathcal{N}$-graphs $\Gamma$ and $\Gamma^{\prime}$ encode the same weakly branched triangulation $(T, \tilde{b})$ if and only if they are equivalent under the relation generated by:

- Plane isotopy.

- Switching the over/under arcs at an accidental crossing.

- The $\mathcal{N}$-graph versions of oriented Reidemeister moves, called $R$-moves (ie formally the usual ones if only accidental crossings are involved, or the Reidemeister move of type III shown in Figure 10 (the orientation being understood) when a dotted crossing is involved). 


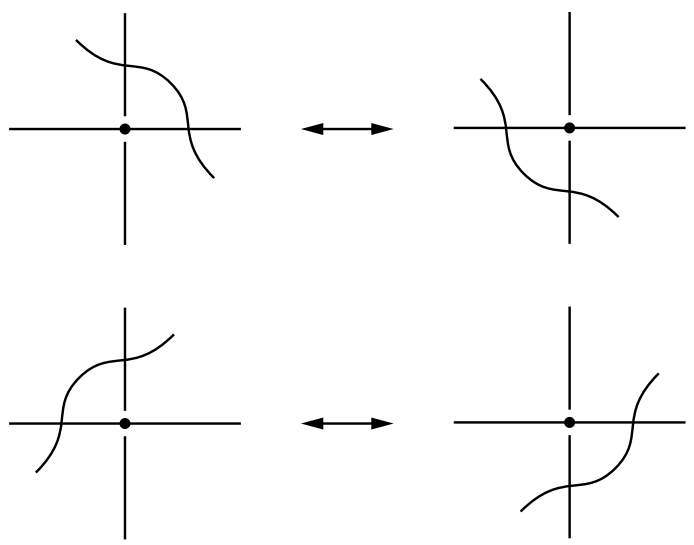

Figure 10: Some Reidemeister moves of type III for $\mathcal{N}$-graphs

\section{B Changing the weak branching}

Given a $\mathcal{N}$-graph $\Gamma$ representing a weakly branched triangulation $(T, \widetilde{b})$, and an arbitrary change of weak branching $(T, \tilde{b}) \rightarrow\left(T, \tilde{b}^{\prime}\right)$, we want to describe a systematic way to modify $\Gamma$ to a $\mathcal{N}$-graph $\Gamma^{\prime}$ representing $\left(T, \tilde{b}^{\prime}\right)$. We begin with the local changes of branching on a given tetrahedron.

6B1 The $\boldsymbol{S}_{\mathbf{4}}$ action $(\boldsymbol{\Delta}, \boldsymbol{b}) \rightarrow\left(\boldsymbol{\Delta}, \boldsymbol{b}_{\boldsymbol{\beta}}\right)$ Consider a branched tetrahedron $(\Delta, b)$ with $b$-ordered vertices $v_{0}, v_{1}, v_{2}, v_{3}$ and 2-facets $F_{0}, F_{1}, F_{2}, F_{3}$, as usual. Let $S\left(J_{4}\right)$ be the symmetric group on the set $J_{4}=\{0,1,2,3\}$. There is a natural one-to-one correspondence between the elements $\beta \in S\left(J_{4}\right)$ and the branchings $b_{\beta}$ on $\Delta$ such that $b=b_{\text {Id }}$. The branching $b$ induces a branching $b(j)$ on each facet $F_{j}$, that is a labeling of the vertices of $F_{j}$ by elements of $J_{3}=\{0,1,2\}$. For every $\beta \in S\left(J_{4}\right)$, also the branching $b_{\beta}$ induces a branching $b_{\beta}(j)$ on $F_{j}$ (note that these facets are still ordered with respect to $b$, not $\left.b_{\beta}\right)$, and the transition from $b(j)$ to $b_{\beta}(j)$ is encoded by a permutation $\epsilon_{\beta}(j) \in S\left(J_{3}\right)$. Clearly the following holds.

Lemma 6.1 (1) The branching signs $*_{b_{\beta}}$ and $*_{b}$ coincide if and only if $\beta \in A\left(J_{4}\right)$.

(2) The 2 -facet $F_{j}$ has the same $b$ - and $b_{\beta}$-transverse coorientations if and only if $\epsilon_{\beta}(j) \in A\left(J_{3}\right)$.

Let $C(b)$ denote a dotted $\mathcal{N}$-graph crossing that encodes $(\Delta, b)$ as in Figure 4 . We call $C(b)$ a 2-2 $\mathcal{N}$-tangle; we consider it as a planar tangle properly embedded in a 2 -disk $D$, with endpoints on $\partial D$ labeled by $J_{4}$. One can assume that the crossing is central to $D$, and that the arcs joining it to the endpoints are contained in two oriented diameters of $D$. We want to describe an algorithm that produces, for every $\beta \in S\left(J_{4}\right)$, a new 
decorated 2-2 $\mathcal{N}$-tangle $C\left(b_{\beta}\right)$ in $D$, with the same unoriented arc-germs as $C(b)$ at $\partial D$. Hence the arcs of $C(b)$ and $C\left(b_{\beta}\right)$ will be in natural one-to-one correspondence and oriented by $b$ and $b_{\beta}$ respectively. The tangle $C\left(b_{\beta}\right)$ will verify the following properties:

- $C\left(b_{\beta}\right)$ contains one dotted crossing. According to the last lemma, $*_{b_{\beta}}=*_{b}$ if and only if $\beta \in A\left(J_{4}\right)$. Hence, given $\beta$, we know the sign $*_{b_{\beta}}$.

- $C\left(b_{\beta}\right)$ can replace $C(b)$ in every $\mathcal{N}$-graph $\Gamma^{\prime}$ obtained after a change of weak branching $\widetilde{b} \rightarrow \widetilde{b}^{\prime}$ acting as $b \rightarrow b_{\beta}$ on the corresponding branched tetrahedron $(\Delta, b)$ of $(T, \tilde{b})$.

- Each of the four arc-germs of $C\left(b_{\beta}\right)$ at $\partial D$ are labeled by an element of $S\left(J_{3}\right)$, which will eventually contribute to the edge coloring of $\Gamma^{\prime}$.

An auxiliary construction Consider the two basic 2-2 tangles in a 2-disk $D^{\prime}$, made by two oriented simple arcs $a, a^{\prime}$ that either are disjoint or intersect transversely at one point, without any over/under information at the crossing point. A $J_{4}$-labeling of the 4 free endpoints of $a \cup a^{\prime}$ is admissible if it respects the following conditions:

(a) The endpoints labeled by 1,3 (resp. 0,2) belong to different arcs and are both either the initial or the final endpoint of the corresponding arc.

(b) 3,2 (resp. 1,0) label the endpoints of the same arc.

These conditions are satisfied by the labels of the tangles of Figure 4; recall that in this figure, $*_{b}=1$ (resp. $*_{b}=-1$ ) if and only if 1, 3 label initial (resp. final) endpoints. By the same rule we give every basic tangle an admissible $J_{4}$-labeling, denoted by $\mathcal{B}$, and a sign $*_{\mathcal{B}}= \pm 1$. Note also that in Figure 4 , the arc with endpoints labeled by 0,1 passes over the other arc. Now we convert $\mathcal{B}$ into a $2-2 \mathcal{N}$-tangle as follows:

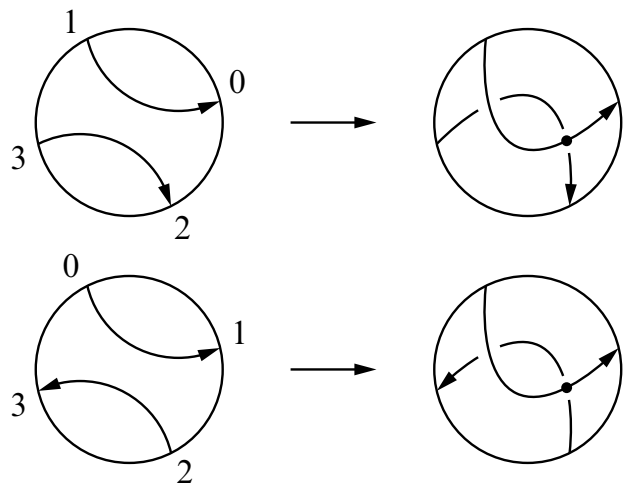

Figure 11: Basic to $\mathcal{N}$-tangles 


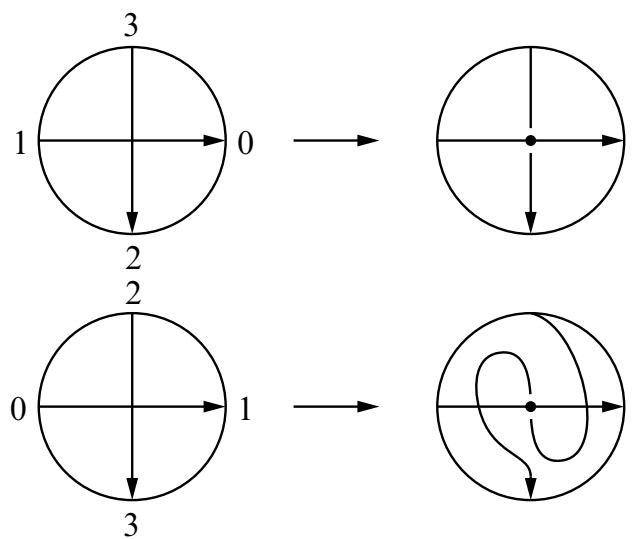

Figure 12: More basic to $\mathcal{N}$-tangles

(c) If $a \cap a^{\prime}=\varnothing$, perform an oriented Reidemeister move of type II of the arc with labels 0,1 over the other arc, thus creating two crossings. Only one of them can be made into a dotted crossing so that its sign $*_{b}$ agrees with $*_{\mathcal{B}}$ (see some examples in Figure 11).

(d) If $a$ and $a^{\prime}$ cross at one point, let $A$ be the arc with 0,1 labels, and $I$ a small open interval in the interior of the arc with 2, 3 labels, such that $I$ contains the crossing point. Let $A$ pass over $I$, put a dot at the crossing point, and turn $I$ so that the crossing sign and $*_{\mathcal{B}}$ agree. Finally complete $I \cup A$ to an $\mathcal{N}$-tangle with the same endpoints as $a \cap a^{\prime}$, by introducing 0 or 2 accidental crossings on opposite sides of $A$ with respect to $I \cap A$ (see some examples in Figure 12).

The algorithm $\boldsymbol{C}(\boldsymbol{b}) \rightarrow \boldsymbol{C}\left(\boldsymbol{b}_{\boldsymbol{\beta}}\right)$ (1) Remove the interior of a smaller concentric subdisk $D^{\prime}$ of $D$. Each endpoint of $C(b)$, say $x_{j}, j \in J_{4}$, is connected by an oriented subarc to a point $x_{\beta(j)}^{\prime}$ on $\partial D^{\prime}$.

(2) There is only one basic 2-2 tangle $\mathcal{B}$ in $D^{\prime}$ such that the $b_{\beta}$-labeling of the endpoints $x_{\beta(j)}^{\prime}$ is admissible and $*_{\mathcal{B}}=*_{b_{\beta}}$. As in the auxiliary construction, convert $\mathcal{B}$ into an 2-2 $\mathcal{N}$-tangle in $D^{\prime}$; glue it to the four subarcs $\left[x_{j}, x_{\beta(j)}^{\prime}\right]$, and denote by $C^{\prime}\left(b_{\beta}\right)$ the resulting $2-2 \mathcal{N}$-tangle in $D$.

(3) The 2-2 $\mathcal{N}$-tangle $C\left(b_{\beta}\right)$ is obtained from $C^{\prime}\left(b_{\beta}\right)$ by giving a label $\widehat{\epsilon}_{\beta}(j) \in S\left(J_{3}\right)$ to each subarc $\left[x_{j}, x_{\beta(j)}^{\prime}\right]$ as follows. Recall the permutation $\epsilon_{\beta}(j) \in S\left(J_{3}\right)$ defined before Lemma 6.1 ; the $b$ - and $b_{\beta}$-orientations of $\left[x_{j}, x_{\beta(j)}^{\prime}\right]$ coincide with each other if and only if $\epsilon_{\beta}(j) \in A\left(J_{3}\right)$ ). Then set

- $\hat{\epsilon}_{\beta}(j)=\epsilon_{\beta}(j)$ if the $b_{\beta}$-orientation of $\left[x_{j}, x_{\beta(j)}^{\prime}\right]$ coincides with the orientation pointing towards the center of $D$;

- $\hat{\epsilon}_{\beta}(j)=\epsilon_{\beta}(j)^{-1}$ otherwise. 
6B2 Iterations Assume that $\beta=\alpha \cdot \gamma$, where by definition $\alpha \cdot \gamma:=\gamma \circ \alpha$. Then we can produce $C\left(b_{\beta}\right)$ by iterating the preceding algorithm twice. We get a sequence of nested disks

$$
D^{\prime \prime} \subset D^{\prime} \subset D,
$$

a subdivision of the segment $\left[x_{j}, x_{\alpha \cdot \gamma(j)}^{\prime \prime}\right]$ into subarcs

$$
\left[x_{j}, x_{\alpha \cdot \gamma(j)}^{\prime \prime}\right]=\left[x_{j}, x_{\alpha(j)}^{\prime}\right] \cup\left[x_{\alpha(j)}^{\prime}, x_{\alpha \cdot \gamma(j)}^{\prime \prime}\right],
$$

and a factorization of the permutation associated to $\left[x_{j}, x_{\alpha \cdot \gamma(j)}^{\prime \prime}\right]$ :

$$
\epsilon_{\beta}(j)=\epsilon_{\alpha}(j) \cdot \epsilon_{\gamma}(\alpha(j)) .
$$

The adjacent transpositions (01), (12), (23) form a standard set of generators of $S_{4}$. Let $\beta=\tau_{1} \cdots \tau_{k}$, where each $\tau_{j}$ is such a generator. Then we can produce again $C\left(b_{\beta}\right)$ by iterating the algorithm $k$ times. We get a sequence of nested disks

$$
D_{k} \subset \cdots \subset D_{1} \subset D,
$$

a subdivision of the $\operatorname{arc}\left[x_{j}, x_{\beta(j)}^{(k)}\right]$ of $C(b) \backslash \operatorname{Int}\left(D_{k}\right)$ into subarcs

$$
\left[x_{j}, x_{\tau_{1}(j)}^{(1)}\right] \cup \cdots \cup\left[x_{\tau_{1} \cdots \tau_{k-1}(j)}^{(k-1)}, x_{\beta(j)}^{(k)}\right],
$$

and a factorization of the permutation $\epsilon_{\beta}(j) \in S_{3}$ associated to the $\operatorname{arc}\left[x_{j}, x_{\beta(j)}^{(k)}\right]$ :

$$
\epsilon_{\beta}(j)=\epsilon_{\tau_{1}}(j) \cdots \epsilon_{\tau_{k}}\left(\tau_{1} \cdots \tau_{k-1}(j)\right)=: f_{1} \cdots f_{k} .
$$

Lemma 6.2 Every transposition $f_{i} \in S_{3}$ belongs to the set $\{$ Id, (01), (12)\}.

Proof It is enough to check the case $\beta=\tau_{j}$ (see Figure 13).

For future application, it is convenient to associate a symbol to each subarc of $C\left(b_{\beta}\right)$, that indicates its label $f \in\{\mathrm{Id},(01),(12)\}$ and depends on its $b$-orientation:

- If the $b$-orientation is outgoing (ie opposite to the one pointing towards the center of $D$ ), then let this symbol be $\angle \operatorname{Id} \angle$, or $\angle f>$ (a source) if $f$ is a transposition.

- If the $b$-orientation is ingoing, then let this symbol be $>$ Id $>$, or $>f \angle$ (a pit) if $f$ is a transposition.

Under concatenation of subarcs, adjacent symbols are separated by $\angle L$ or $>>$. Here are examples:

$$
\begin{aligned}
& \cdots \angle f>>g \angle \cdots, \quad \cdots>f \angle \angle g>\cdots, \quad \cdots>\operatorname{Id} \triangle>\operatorname{Id} \triangle \cdots, \\
& \cdots \angle \operatorname{Id} \angle \angle \operatorname{Id} \angle \cdots, \quad \cdots>\operatorname{Id} \triangle \triangle f \angle \cdots, \quad \cdots \angle \operatorname{Id} \angle \angle f>\cdots \text {, etc. }
\end{aligned}
$$


Letting $? \in\{L L, \triangle \supset\}$, in the case of $\epsilon_{\beta}(j)=f_{1} \cdots f_{k}$ we get:

- $\angle f_{1} ? \cdots ? f_{k} \angle$ or $>f_{1}$ ? $\cdots ? f_{k}>$ if $b$ and $b_{\beta}$ induce on $\left[x_{j}, x_{\beta(j)}^{(k)}\right]$ the same orientation.

- $\angle f_{1} ? \cdots ? f_{k}>$ or $>f_{1}$ ? $\cdots ? f_{k} \angle$ otherwise.

We can also make simplifications of the form

$$
? s>>f \angle \angle f>>t ?=? s>>t ?
$$

and so on, so that the concatenation of symbols of subarcs eventually reduces to $\triangle \operatorname{Id} \triangle, \angle \mathrm{Id} \angle$, or alternating pits and sources carrying the transpositions (01) and (12). In Figure 13 we show graphically the result when $*_{b}=+1$ and $\beta$ varies in $\{(01),(12),(23)\}$; we dropped the notation Id for the symbol $\supset$ Id $\supset$. The case $*_{b}=-1$ is similar.

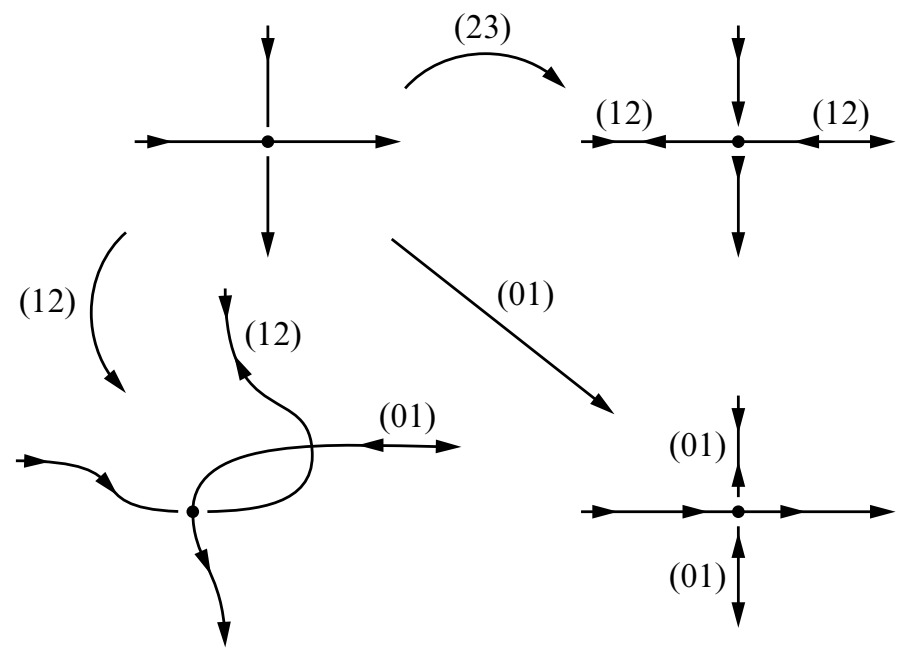

Figure 13: $C\left(b_{(i j)}\right), *_{b}=+1$

6B3 Oriented $C$-moves We call $C(b) \rightarrow C\left(b_{\beta}\right)$ a $C$-move. If $C(b)$ and $C\left(b_{\beta}\right)$ define the same prebranching then we say that it is an oriented $C-$ move. Set

$$
\sigma=(0123)=(23) \cdot(12) \cdot(01), \quad \tau=(02) \cdot(13) .
$$

Then $\sigma$ generates a subgroup $\mathfrak{G}$ of $S\left(J_{4}\right)$ isomorphic to $(\mathbb{Z} / 4 \mathbb{Z},+)$ via the map $\sigma \mapsto 1$.

Lemma 6.3 For both $*_{b}= \pm 1, \beta \in S_{4}$ induces an oriented $C$-move $C(b) \rightarrow C\left(b_{\beta}\right)$ if and only if $\beta \in \mathfrak{G}$. 
Proof Looking at Figure 2 anticlockwise from the top left, and denoting by $b$ the first branching (so that $*_{b}=+1$ ), the others are successively $b_{\tau}, b_{\sigma \cdot \tau}, b_{\tau \cdot \sigma \cdot \tau}, b_{\sigma^{-1} \cdot \cdot \cdot \tau \cdot \tau}=b$. Hence $\beta \in \mathfrak{G}$. One can do similarly when $*_{b}=-1$.

Figure 14 shows the oriented $C$-moves $C(b) \rightarrow C\left(b_{\sigma^{-1}}\right)$ for $*_{b}=+1$ and $C(b) \rightarrow$ $C\left(b_{\sigma}\right)$ for $*_{b}=-1$.

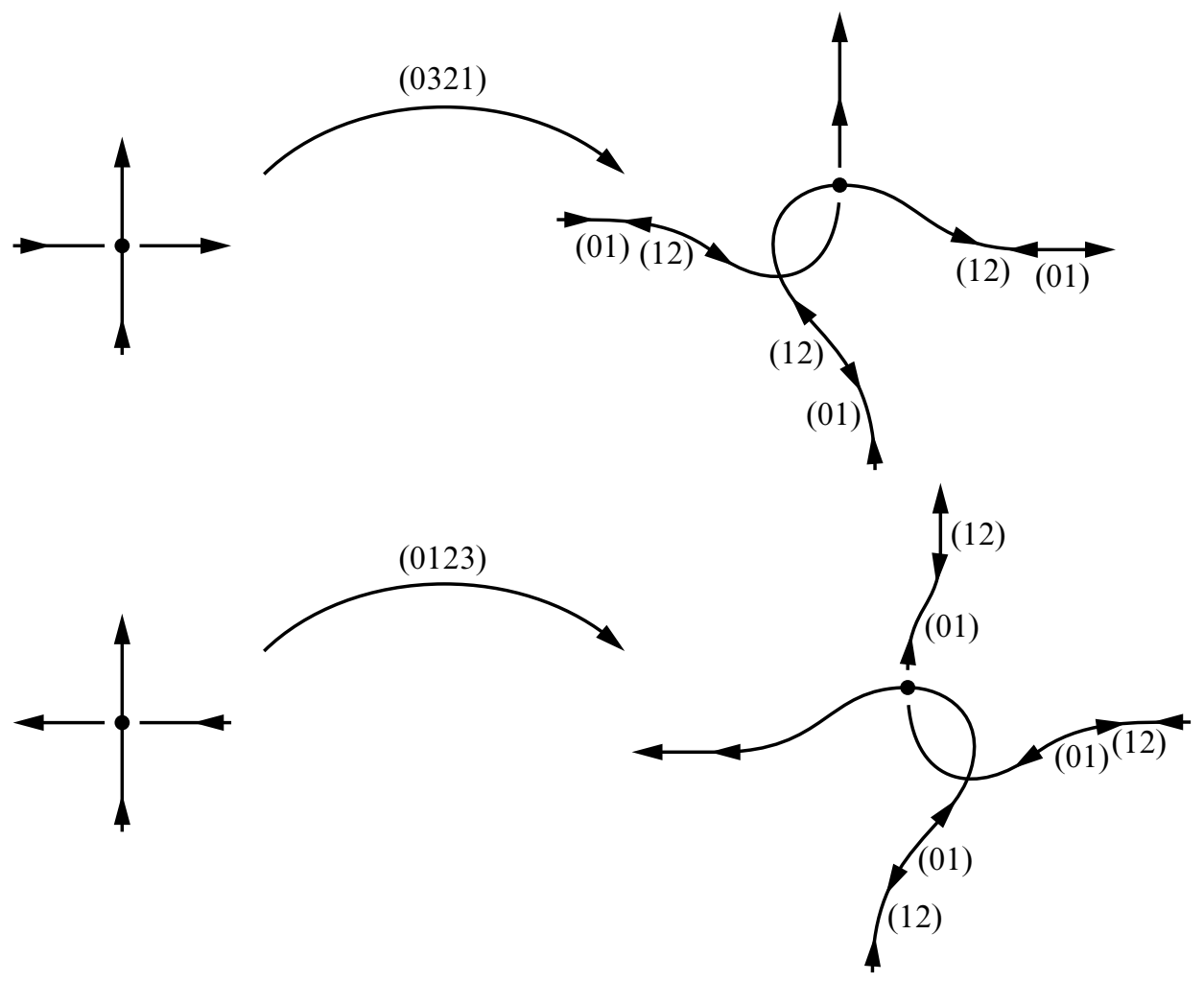

Figure 14: Generating oriented $C$-moves

6B4 Globalization Let $\Gamma$ be an $\mathcal{N}$-graph encoding $(T, \tilde{b})$, and $\widetilde{b}^{\prime}$ another weak branching on $T$. We want to produce an $\mathcal{N}$-graph $\Gamma^{\prime}$ encoding $\left(T, \tilde{b}^{\prime}\right)$. For every abstract branched tetrahedron $\left(\Delta_{j}, b_{j}\right)$ of $(T, \widetilde{b})$ there is a permutation $\beta_{j} \in S\left(J_{4}\right)$ such that transit $\tilde{b} \rightarrow \widetilde{b}^{\prime}$, restricted to to $\Delta_{j}$, is $b_{j} \rightarrow\left(b_{j}\right)_{\beta_{j}}$. Applying at every crossing of $\Gamma$ the algorithm $C\left(b_{j}\right) \rightarrow C\left(\left(b_{j}\right)_{\beta_{j}}\right)$, we get a diagram $\Gamma^{\prime}$. Forgetting the orientations, there is a natural one-to-one correspondence between the edges of $\Gamma$ and $\Gamma^{\prime}$. Let $e$ be an edge of $\Gamma$ with color $r(e)$. Denote by $\bar{e}$ the corresponding edge of $\Gamma^{\prime}$. According to the last step of the algorithm, $\bar{e}$ is labeled by "initial" and "final" permutations $\hat{\epsilon}^{i}(\bar{e}), \hat{\epsilon}^{f}(\bar{e}) \in S\left(J_{3}\right)$. There is an alternative: 
- Both $\hat{\epsilon}^{i}(\bar{e})$ and $\hat{\epsilon}^{f}(\bar{e})$ belong to $A\left(J_{3}\right)$ (in such a case $e$ and $\bar{e}$ have compatible orientations); then the color $r(\bar{e})$ is defined by

$$
\hat{\epsilon}^{i}(\bar{e}) \cdot(012)^{r(e)} \cdot \hat{\epsilon}^{f}(\bar{e})=(012)^{r(\bar{e})} .
$$

- Both $\hat{\epsilon}^{i}(\bar{e})$ and $\hat{\epsilon}^{f}(\bar{e})$ do not belong to $A\left(J_{3}\right)$ (in such a case $e$ and $\bar{e}$ have opposite orientations); then the color $r(\bar{e})$ is defined by

$$
\hat{\epsilon}^{i}(\bar{e}) \cdot(012)^{-r(e)} \cdot \hat{\epsilon}^{f}(\bar{e})=(012)^{r(\bar{e})} .
$$

The oriented $C$-moves have the nice feature that they are local and independent of each other. On the contrary, a family of permutations $\beta_{j} \in S\left(J_{4}\right)$ as above must satisfy nontrivial global constraints to induce a change of weak branching $\widetilde{b} \rightarrow \widetilde{b}^{\prime}$. Fortunately, the following local/global calculus on $\mathcal{N}$-graphs from [9] covers the general case.

Lemma 6.4 Any change of $\mathcal{N}$-graphs $\Gamma \rightarrow \Gamma^{\prime}$ corresponding to a change of weak branching $(T, \widetilde{b}) \rightarrow\left(T, \widetilde{b}^{\prime}\right)$ is a composition of a finite sequence of the following moves $\Gamma_{j} \rightarrow \Gamma_{j+1}$ :

(1) The moves of Section $6 A$.

(2) The oriented $C$-moves.

(3) A nonlocal circuit move: Let $\gamma$ be a simple circuit of $\Gamma_{j}$, ie a circuit with one component. Assume that each edge of $\gamma$ is the upper strand at each crossing through which $\gamma$ passes. Then $\Gamma_{j+1}$ is obtained by acting on each of these crossings with the transposition $(23) \in S_{4}$. Equivalently, $\Gamma_{j+1}$ is obtained by reversing the orientation of $\gamma$ and keeping the $\mathbb{Z} / 3 \mathbb{Z}$-color $r(e)$ unchanged at every edge $e$ of $\operatorname{Sing}(P)$.

Proof The equivalence between the two descriptions of the nonlocal circuit move is clear. Let $\Gamma \rightarrow \Gamma^{\prime}$ be as in the statement. The set $\mathcal{S}$ of edges of $\operatorname{Sing}(P)$ where the prebranchings associated to $\tilde{b}$ and $\tilde{b}^{\prime}$ disagree is the union of nonoverlapping simple circuits $\gamma_{1}, \ldots, \gamma_{r}$ oriented by $\tilde{b}$. Using oriented $C$-moves one can modify $\Gamma$ so that $\gamma_{1}$ eventually passes over at each crossing where it goes through. Then change its orientation. Doing similarly with $\gamma_{2}, \ldots, \gamma_{r}$, one ends up with $\mathcal{S}=\varnothing$.

\section{C Transits}

It is well known that two naked triangulations $T$ and $T^{\prime}$ of $\widehat{V}$ can be connected by a finite sequence of 3-dimensional Pachner's moves, also called MP and bubble moves. The MP moves, also called $2 \leftrightarrow 3$ moves, are illustrated in Figure 15 in terms of triangulations and dual spines. Branched versions of the bubble moves are shown in Figure 16 in terms of $\mathcal{N}$-graphs. 

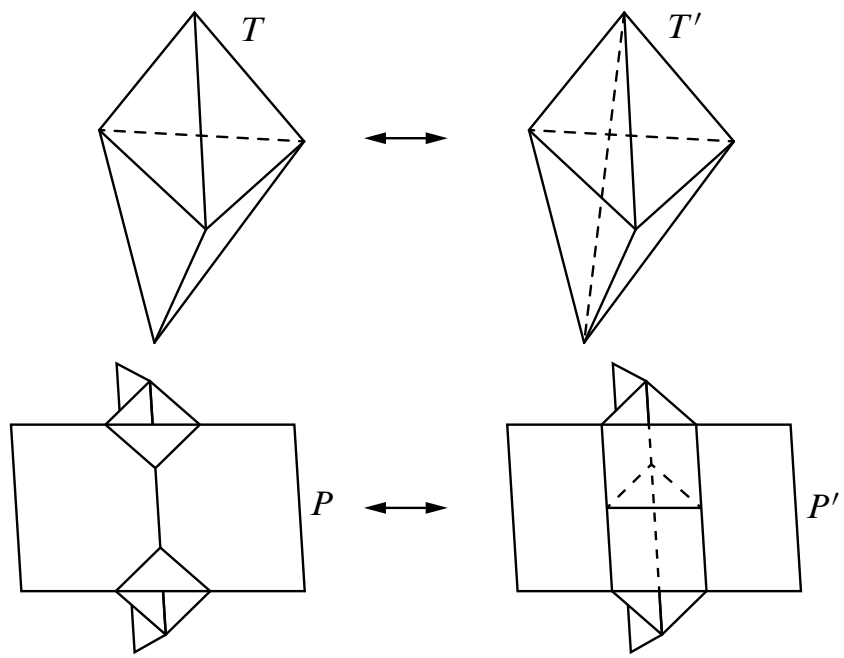

Figure 15: The MP move
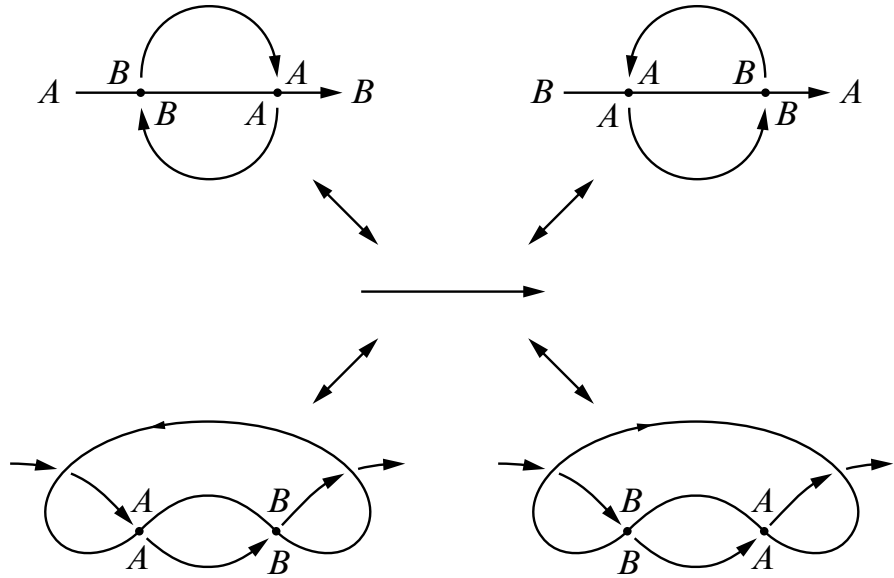

Figure 16: Bubble $b$-transits

Definition 6.5 Let $(T, \sigma)$ and $\left(T^{\prime}, \sigma^{\prime}\right)$ be prebranched triangulations of $\hat{V}$. A move $T \rightarrow T^{\prime}$ (either bubble or MP, positive or negative) lifts to a prebranching transit $(T, \sigma) \rightarrow\left(T^{\prime}, \sigma^{\prime}\right)$ if and only if $\sigma$ and $\sigma^{\prime}$ coincide on every common 2-face of $T$ and $T^{\prime}$. We say that $T \rightarrow T^{\prime}$ lifts to a $\tilde{b}$-transit $(T, \tilde{b}) \rightarrow\left(T^{\prime}, \tilde{b}^{\prime}\right)$ of weakly branched triangulations if it induces a prebranching transit $(T, \sigma \widetilde{b}) \rightarrow\left(T^{\prime}, \sigma \widetilde{b}^{\prime}\right)$. If $(T, b)$ and $\left(T^{\prime}, b^{\prime}\right)$ are branched triangulations, then $(T, b) \rightarrow\left(T^{\prime}, b^{\prime}\right)$ is a $b$-transit if $b$ and $b^{\prime}$ coincide on every common edge of $T$ and $T^{\prime}$. 
Figure 16 shows the different bubble $b$-transits in terms of normal $\mathcal{N}$-graphs (recall Remark 2.11), and the decorations $A, B$ of the dual square edges according to Figure 8. Figure 17 and 18 show an example of MP $b$-transit in terms of branched triangulations and normal $\mathcal{N}$-graphs; note that $*_{b}=+1$ for all 3 -simplices (resp. dotted crossings). It is called the Schaeffer $b$-transit, and plays a distinguished role in the study of matrix dilogarithms (see Section 8).
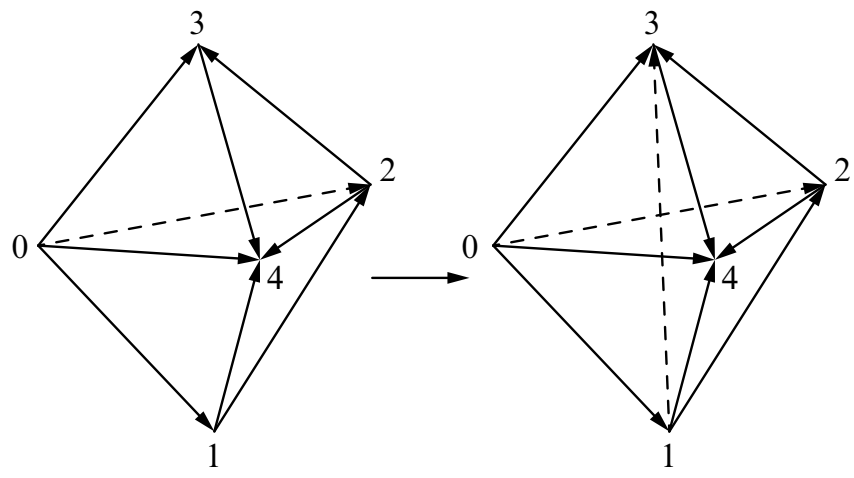

Figure 17: Schaeffer's $b$-transits
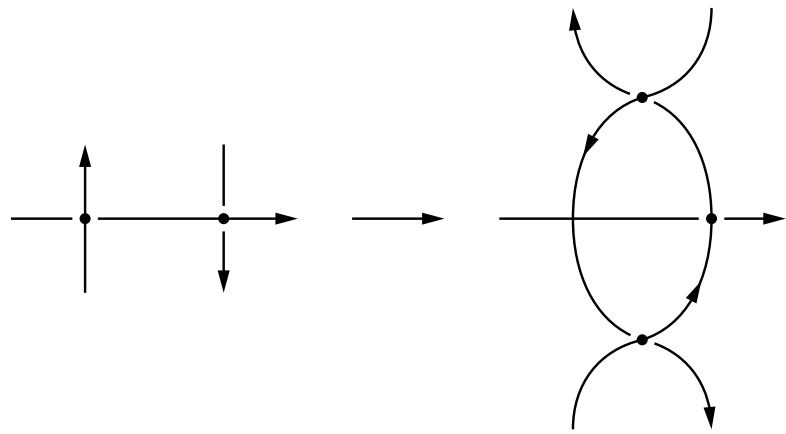

Figure 18: Normal $\mathcal{N}$-graph Schaeffer's $b$-transit

The following lemma will be useful in applications to the $\mathrm{QH}$ state sums. Denote by $\cong p b$ the equivalence relation on the set of weakly branched triangulations, defined by $\widetilde{b}$-transits $(T, \tilde{b}) \rightarrow\left(T, \tilde{b}^{\prime}\right)$.

Lemma 6.6 The equivalence relation $\cong_{p b}$ is generated by the following two transformations:

- Change the weak-branching $(T, \tilde{b}) \rightarrow\left(T, \tilde{b}^{\prime}\right)$ by moves preserving the prebranching (ie dually do oriented $C$-moves, see Lemma 6.4).

- Perform $\tilde{b}$-transits $(T, \tilde{b}) \rightarrow\left(T^{\prime}, \tilde{b}^{\prime}\right)$ which look like $b$-transits at the subpatterns of tetrahedra involved in the move. 

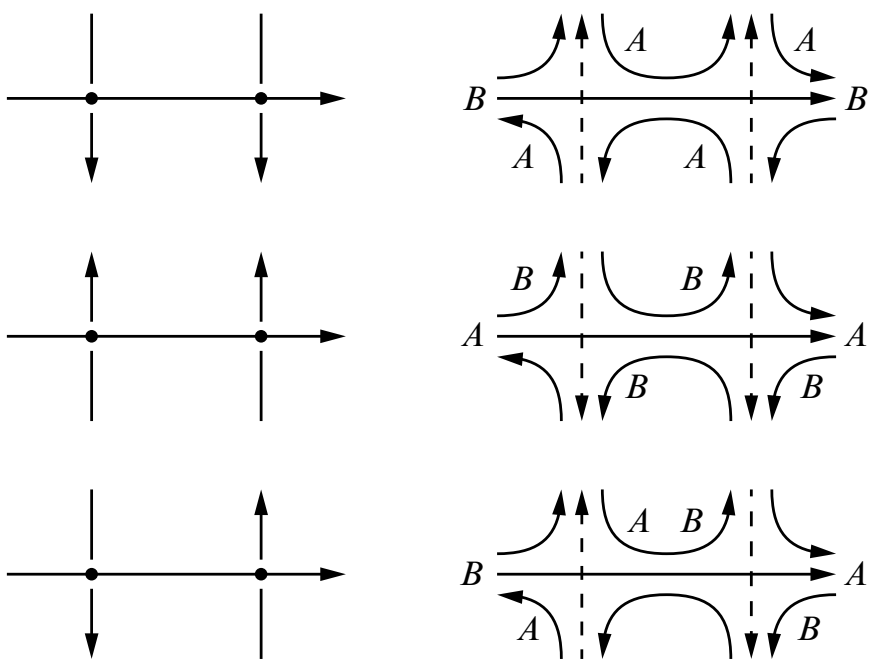

Figure 19: Branched realization of MP $p b$-transits

Proof The proof for bubble moves is easy and basically illustrated in Figure 16 in terms of $\mathcal{N}$-graphs. Consider a positive MP $\tilde{b}$-transit $(T, \tilde{b}) \rightarrow\left(T^{\prime}, \widetilde{b}^{\prime}\right)$, and the two tetrahedra of $T$ modified by the transit. At their common 2 -face $F$ one of the following possibilities is realized:

(1) There are exactly two square edges which are both not monochromatic (ie each one inherits different $A, B$ labels from the prebranchings of the two tetrahedra).

(2) There is exactly one square edge which is monochromatic (either $A$ or $B$ ), and the other two edges are monochromatic (either $B$ or $A$ coupled with the empty label).

Moreover, this information at $F$ determines completely the coorientations of the three 2-faces produced by the transit, and hence determines the prebranching transit $(T, \sigma \widetilde{b}) \rightarrow\left(T^{\prime}, \sigma \widetilde{b}^{\prime}\right)$. Then, it is enough to realize the above possibilities by means of $b$-transits (changing the weak branchings before and/or after the transit, if necessary, by moves preserving the prebranching). Figure 19 shows it in terms of (decoded) normal $\mathcal{N}$-graphs. Clearly this realization is not unique.

\section{State sum invariance over weakly branched triangulations}

Let $(T, \tilde{b}, w, f, c)$ be a weakly branched $\mathrm{QH}$ triangulation of a pattern $\mathcal{P} ; w$ and $f$ satisfy the defining equations of the spaces in Definition 3.7, and $c$ those of Definition 4.7 (resp. Definition 5.1) if $\mathcal{P}$ is a pattern over a cusped manifold (resp. a QHFT pattern). 
We want to prove that $\mathcal{H}_{N}(\mathcal{P}): \equiv_{N} \pm \mathcal{H}_{N}(T, \tilde{b}, w, f, c)$ is an invariant of $\mathcal{P}$. In the case when $(T, b)$ is a branched triangulation we achieved this result in [3; 4; 5] by introducing the notion of $Q H$ transit $(T, b, w, f, c) \rightarrow\left(T^{\prime}, b^{\prime}, w^{\prime}, f^{\prime}, c^{\prime}\right)$. A QH transit is such that: $(T, b) \rightarrow\left(T^{\prime}, b^{\prime}\right)$ is a $b$-transit, the tuples $(w, f, c)$ and $\left(w^{\prime}, f^{\prime}, c^{\prime}\right)$ coincide on the common tetrahedra of $T$ and $T^{\prime}$, and for every common edge $e$ we have (see Section 3B for the notations, where $W_{T}(e)$ is $W(e)$, and so on)

$$
W_{T}(e)=W_{T^{\prime}}(e), \quad L_{T}(e)=L_{T^{\prime}}(e), \quad C_{T}(e)=C_{T^{\prime}}(e) .
$$

$\mathrm{QH}$ triangulations related by $\mathrm{QH}$ transits encode a same pattern $\mathcal{P}$. Moreover:

- $\mathcal{H}_{N}(T, b, w, f, c)$ is invariant under any $\mathrm{QH}$ transit.

- Any two branched $\mathrm{QH}$ triangulations of $\mathcal{P}$ are related by a finite sequence of $\mathrm{QH}$ transits and branching changes.

- $\mathcal{H}_{N}(T, b, w, f, c)$ is invariant under any change of the branching $b$.

Clearly these three facts prove the invariance of $\mathcal{H}_{N}(\mathcal{P})$. We follow the same strategy when $\widetilde{b}$ is an arbitrary weak branching. Since the $\mathrm{QH}$ transits are local transformations, we define QH transits $(T, \tilde{b}, w, f, c) \rightarrow\left(T, \tilde{b}^{\prime}, w^{\prime}, f^{\prime}, c^{\prime}\right)$ by requiring that the $\tilde{b}$-transit $(T, \tilde{b}) \rightarrow\left(T^{\prime}, \widetilde{b}^{\prime}\right)$ restricts to a genuine $b$-transit on the tetrahedra modified by the move. Then, trivially $\mathcal{H}_{N}(T, \tilde{b}, w, f, c)$ is again invariant under $\mathrm{QH}$ transits, and using Lemma 6.6 and the results of $[3 ; 4 ; 5]$ it is easy to see that any two weakly branched $\mathrm{QH}$ triangulations of $\mathcal{P}$ are related by a finite sequence of $\mathrm{QH}$ transits and changes of weak branching. So it remains to show:

Proposition 7.1 For any change of weak branching $\tilde{b} \rightarrow \tilde{b}^{\prime}$ we have

$$
\mathcal{H}_{N}(T, \tilde{b}, w, f, c) \equiv_{N} \pm \mathcal{H}_{N}\left(T, \tilde{b}^{\prime}, w, f, c\right) .
$$

In order to develop the proof we need to fix some conventions regarding the contraction of tensors, and to describe the relations between tetrahedral tensors related by a change of branching. This is the content of the two following subsections.

\section{A Formal conversion of tensor networks}

Recall the notation $V=\mathbb{C}^{N}$. We use the canonical isomorphism $V \rightarrow V^{*}$ sending the standard basis to the dual basis. Let $A \in \operatorname{End}\left(\mathbb{C}^{N} \otimes \mathbb{C}^{N}\right)$ be associated to a 2-2 $\mathcal{N}$-tangle as in Figure 9, according to the conventions of Section 3A. Let $\beta \in S\left(J_{4}\right)$. Assuming that $*_{b}=1$, under the natural isomorphisms

$$
\operatorname{Hom}\left(V_{3} \otimes V_{1}, V_{2} \otimes V_{0}\right) \cong V_{2} \otimes V_{0} \otimes\left(V_{3} \otimes V_{1}\right)^{*} \cong V_{2} \otimes V_{0} \otimes V_{1}^{*} \otimes V_{3}^{*}
$$


we see that $A$ belongs to $V_{2} \otimes V_{0} \otimes V_{1}^{*} \otimes V_{3}^{*}$, where as usual $V_{j}$ is the copy of $\mathbb{C}^{N}$ associated to the $j^{\text {th }}$ face of $(\Delta, b)$. Consider $W_{2} \otimes W_{0} \otimes W_{1}^{*} \otimes W_{3}^{*}$ if $* b_{\beta}=1$, and $W_{3} \otimes W_{1} \otimes W_{0}^{*} \otimes W_{2}^{*}$ if $* b_{\beta}=-1$, where $W_{j}$ is the copy of $\mathbb{C}^{N}$ associated to the $j^{\text {th }}$ face according to $b_{\beta}$. By using Id: $V_{j} \rightarrow W_{\beta(j)}$ or the canonical isomorphisms $V_{j} \rightarrow W_{\beta(j)}^{*}$, we get further canonical isomorphisms

$$
\begin{aligned}
& V_{2} \otimes V_{0} \otimes V_{1}^{*} \otimes V_{3}^{*} \cong W_{2} \otimes W_{0} \otimes W_{1}^{*} \otimes W_{3}^{*}, \\
& V_{2} \otimes V_{0} \otimes V_{1}^{*} \otimes V_{3}^{*} \cong W_{1} \otimes W_{3} \otimes W_{0}^{*} \otimes W_{2}^{*} .
\end{aligned}
$$

Denote by $A_{\beta}$ the operator supported by $\left(\Delta, b_{\beta}\right)$, defined as the image of $A$ via such an isomorphism. We call $A_{\beta}$ the formal $\beta$-conversion of $A$. There are explicit identities between the matrix elements of $A$ and $A_{\beta}$. Following our conventions for the index positions, we get for example

$$
A_{s, t}^{p, q}=\left(A_{(13)(02)}\right)_{t, s}^{q, p}=\left(A_{(23)}\right)_{s, q}^{p, t}=\left(A_{(02)}\right)_{q, p}^{s, t}=\left(A_{(01)}\right)_{p, t}^{s, q}=\left(A_{(12)}\right)_{t, q}^{s, p} .
$$

Let $\mathcal{A}$ be a $\mathrm{QH}$ tensor network supported by a weakly branched triangulation $(T, \tilde{b})$. For any change of weak branching $\widetilde{b} \rightarrow \widetilde{b}^{\prime}$, the formal conversions of the tetrahedral tensors of $\mathcal{A}$ match to produce a new tensor network $\mathcal{A}{\widetilde{b^{\prime}}}^{\prime}$, supported by $\left(T, \tilde{b}^{\prime}\right)$. The state sums associated to $\mathcal{A}$ and $\mathcal{A} \widetilde{b}^{\prime}$ being total contractions, it is tautologically evident that they take the same values.

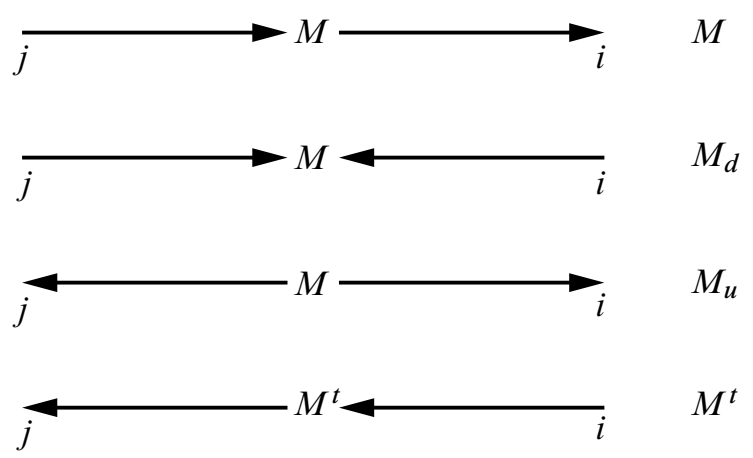

Figure 20: Formal conversion of a square matrix

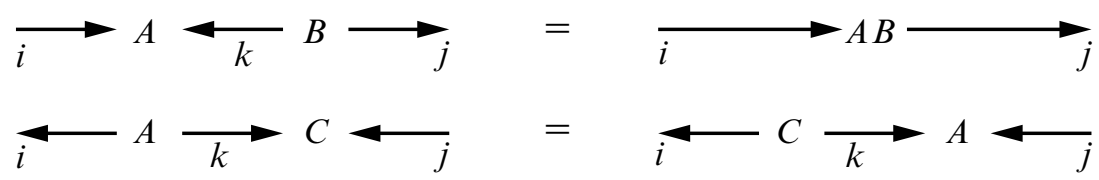

Figure 21: Relations for $M$-conversion

The formal conversion of tensors can be widely applied. Here is a second example that we use below. Let $M=\left(M_{j}^{i}\right)$ be any $N \times N$ matrix. We interpret $M$ as an 
endomorphism of $V=\mathbb{C}^{N}$. There are two formal conversions of $M, M_{u}$ and $M_{d}$, which are bilinear forms on $V^{*}$ and on $V$ respectively, defined by:

$$
\left(M_{u}\right)^{i, j}=M_{j}^{i}=\left(M_{d}\right)_{i, j} .
$$

Note that we have also $\left(M_{u}\right)^{i, j}=\left(M^{t}\right)_{i}^{j}=\left(M_{d}\right)_{i, j}$, where $M^{t}$ is the transpose matrix of $M$, that is, the adjoint endomorphism. Figure 20 shows a graphical encoding of these identities. If $M$ is symmetric, the position of the indices $i, j$ is immaterial. Such formal conversions satisfy a few relations shown in Figure 21, where $A, B$ and $C$ denote $N \times N$ matrices and we assume that $A C=C A$. Note that in every case the index $k$ is traced out, and the top/right picture represents the matrix element of a composite endomorphism

$$
A \cdot B:=B \circ A \text {. }
$$

\section{B The relations between $\mathcal{R}_{N}(\Delta, b, w, f, c)$ and $\mathcal{R}_{N}\left(\Delta, b_{\beta}, w^{\prime}, f^{\prime}, c^{\prime}\right)$}

Consider a change of branching $b \rightarrow b_{\beta}, \beta \in S\left(J_{4}\right)$. It is enough to treat the case where $\beta$ is one of the standard generators $(01),(12),(23) \in S\left(J_{4}\right)$. Consider the algorithm $C(b) \rightarrow C\left(b_{\beta}\right)$ of Section 6B. Associate a tensor network to $C\left(b_{\beta}\right)$ as follows:

- Associate $\mathcal{R}_{N}\left(\Delta, b_{\beta}, w^{\prime}, f^{\prime}, c^{\prime}\right)$ to the dotted crossing of $C\left(b_{\beta}\right)$.

- Using the matrices $S$ and $T$ of Section 3B, replace $\triangle(01) \angle$ with $\triangle T \angle$, $>(12) \angle$ with $\triangle S \angle, \angle(01)>$ with $\angle T^{-1}>, \angle(12)>$ with $\angle S^{-1}>$, and $>$ Id $\supset$ with $>I>$.

Contracting the tensor network we get a tensor $\widehat{\mathcal{R}}_{N}\left(T, b_{\beta}, w^{\prime}, f^{\prime}, c^{\prime}\right)$ which has the same type as $\mathcal{R}_{N}(\Delta, b, w, f, c)$, since both have the same oriented arc-germs at $\partial D$. The next proposition rephrases the content of [3, Lemma 3.3] and [4, Corollary 5.6]. Matrix indices are raised or lowered using the standard inner product on $\mathbb{C}^{N}$ (see Section 7A).

Proposition 7.2 If $\beta=(01)$, (12), (23), then

$$
\mathcal{R}_{N}(T, b, w, f, c) \equiv_{N} \pm \widehat{\mathcal{R}}_{N}\left(T, b_{\beta}, w^{\prime}, f^{\prime}, c^{\prime}\right) .
$$

More precisely, exchanging the roles of $b$ and $b_{\beta}$ and setting $\epsilon_{N}=(-1)^{(N-1) / 2}$, we have

$$
\begin{aligned}
& \mathcal{R}_{N}\left(\Delta, b_{(01)}, w^{\prime}, f^{\prime}, c^{\prime}\right)_{k, j}^{i, l} \equiv_{N} \epsilon_{N}^{c_{0}} T_{k, k^{\prime}} \mathcal{R}_{N}(\Delta, b, w, f, c)_{i^{\prime}, j}^{k^{\prime}, l}\left(T^{-1}\right)^{i^{\prime}, i}, \\
& \mathcal{R}_{N}\left(\Delta, b_{(12)}, w^{\prime}, f^{\prime}, c^{\prime}\right)_{j, l}^{i, k} \equiv_{N} \epsilon_{N}^{c_{1}} T_{l, l^{\prime}} \mathcal{R}_{N}(\Delta, b, w, f, c)_{i^{\prime}, j}^{k, l^{\prime}}\left(S^{-1}\right)^{i^{\prime}, i}, \\
& \mathcal{R}_{N}\left(\Delta, b_{(23)}, w^{\prime}, f^{\prime}, c^{\prime}\right)_{i, l}^{k, j} \equiv_{N} \epsilon_{N}^{c_{0}} S_{l, l^{\prime}} \mathcal{R}_{N}(\Delta, b, w, f, c)_{i, j^{\prime}}^{k, l^{\prime}}\left(S^{-1}\right)^{j^{\prime}, j} .
\end{aligned}
$$


The main properties of $S$ and $T$ are described in the following lemma.

\section{Lemma 7.3 (1) $S^{4}=\mathrm{I}_{N}$.}

(2) $(T S)^{3}=\phi_{N} S^{2}$, where $\phi_{N} \in\{ \pm 1, \pm i\}$ is given by

$$
\phi_{N}=\left\{\begin{array}{ccc}
\left(\frac{m+1}{N}\right) & \text { if } N \equiv 1 \quad \bmod 4, \\
i\left(\frac{m+1}{N}\right) & \text { if } N \equiv 3 \bmod 4 .
\end{array}\right.
$$

(3) $\left(S^{-1} T\right)^{3}=\phi_{N} \mathrm{I}_{N}$.

Proof (1) is immediate. We have $\left((T S)^{2}\right)_{i}^{j}=N^{-1} \zeta^{-(m+1) i^{2}-i j} \sum_{k=0}^{N-1} \zeta^{(m+1)(k-i-j)^{2}}$. For all nonvanishing coprime integers $a, b$ with $b>0$ set

$$
G(a, b)=\sum_{x} e^{2 \pi i a x^{2} / b} .
$$

The sum in $\left((T S)^{2}\right)_{i}^{j}$ has this form for $a=m+1, b=N$. By [26, pages 86-87] we have

$$
G(a, b)=\left(\frac{a}{b}\right) G(1, b), \quad b \text { odd },
$$

where $G(1, b)=\sqrt{b}$ if $b \equiv 1 \bmod 4$, and $G(1, b)=i \sqrt{b}$ if $b \equiv 3 \bmod 4$. Hence

$$
\left((T S)^{2}\right)_{i}^{j}=\phi_{N} N^{-1 / 2} \zeta^{-(m+1) i^{2}-i j},
$$

and (2) follows easily from this. As $T S^{-1}=S\left(S^{-1} T\right) S^{-1}$, it is enough to prove the last statement for $T S^{-1}$. We have

$$
(T S)^{4}=(T S)(T S)^{3}=\phi_{N} T S^{3}=\phi_{N} T S^{-1}, \quad\left(\phi_{N} T S^{-1}\right)^{3}=\left((T S)^{4}\right)^{3}=\phi_{N}^{4} \mathrm{I}_{N}
$$

and finally $\left(T S^{-1}\right)^{3}=\phi_{N} \mathrm{I}_{N}$.

Proof of Proposition 7.1 By Lemma 6.4 we can assume that $(T, \tilde{b}) \rightarrow\left(T, \tilde{b}^{\prime}\right)$ corresponds to an oriented $C$-move or a circuit move on the associated $\mathcal{N}$-graphs. A circuit move preserves the $\mathbb{Z} / 3 \mathbb{Z}$-color $r(e)$ of every edge $e$, and produces one extremal pit and one extremal source associated to the matrices $S$ and $S^{-1}$ at the endpoints of each edge of the circuit. Hence $\mathcal{H}_{N}(T, \tilde{b}, w, f, c)$ is preserved, as it is the contraction of a tensor network. Consider the generating oriented $C$-move associated to $\sigma=(0123) \in S\left(J_{4}\right)$, and the tensor $\widehat{\mathcal{R}}_{N}\left(T, b_{\sigma}, w^{\prime}, f^{\prime}, c^{\prime}\right)$ resulting from the formal conversion of the sequence

$$
C(b) \rightarrow C\left(b_{(23)}\right) \rightarrow C\left(b_{(23)(12)}\right) \rightarrow C\left(b_{(23)(12)(01)}\right)=C\left(b_{\sigma}\right)
$$


as before Proposition 7.2. From Figure 14 we see that an edge $e^{\prime}$ of $C\left(b_{\sigma}\right)$ carries the matrix $\mathcal{Q}_{N}=S \cdot T^{-1}$ if $r\left(e^{\prime}\right)=1+r(e) \in \mathbb{Z} / 3 \mathbb{Z}$ ( $e$ being the arc of $C(b)$ associated to $\left.e^{\prime}\right)$, and that $e^{\prime}$ carries $\mathcal{Q}_{N}^{-1}=T \cdot S^{-1}$ if $r\left(e^{\prime}\right)=-1+r(e) \in \mathbb{Z} / 3 \mathbb{Z}$. Otherwise it carries the identity matrix. By the relation $\mathcal{Q}_{N}^{3}=\phi_{N}^{-1} \mathrm{I}_{N}$ proved in Lemma 7.3, the sum in $\mathcal{H}_{N}(T, \widetilde{b}, w, f, c)$ is changed by a factor $\pm i$ when $r\left(e^{\prime}\right)=1+r(e)$ and $r(e)=2$, or when $r\left(e^{\prime}\right)=-1+r(e)$ and $r(e)=0$. The term $\phi_{N}^{-q(T, \tilde{b})}$ of $a_{N}(T, \tilde{b})$ compensates exactly such factors.

\section{Resolution of the sign anomaly}

In this Section we prove Theorem 1.1(3) and the analogous result for QHFT partition functions:

Proposition 8.1 Let $\mathcal{P}$ be a QHFT pattern or a pattern over a cusped manifold. If $N-1 \equiv 0 \bmod 4$, or $N-1 \equiv 2 \bmod 4$ and the bulk $c$-weight of $\mathcal{P}$ vanishes, then $\mathcal{H}_{N}(\mathcal{P})$ is defined up to multiplication by $N^{\text {th }}$ roots of unity.

Proof We combine several results described below in Sections $8 \mathrm{~A}$ and $8 \mathrm{~B}$. By Corollary 8.6, for any odd $N \geq 3$ the invariance of $\mathcal{H}_{N}(\mathcal{T})$ under QH transits holds true up to multiplication by $N^{\text {th }}$ roots of unity. If $N-1 \equiv 0 \bmod 4$, we have $\epsilon_{N}=1$, so $\mathcal{H}_{N}(\mathcal{T})$ has no sign anomaly with respect to any change of weak branching (Proposition 7.2). If $N-1 \equiv 0 \bmod 4$ and $h_{c}=0$, the same is true by Lemma 8.7 and 8.8. Then the conclusion follows as explained before Proposition 7.1

Let $\mathcal{P}$ be a pattern for which $\mathcal{H}_{N}(\mathcal{P})$ can be defined by using branched triangulations, as in $[3 ; 4 ; 5]$. Then $a_{N}(T, b)=N^{-v}$ or 1 , so we can wonder if the sign anomalies occurring in Proposition 7.2 disappear because of global compensations. This is eventually true.

Proposition 8.2 For any pattern $\mathcal{P}$ as above, $\mathcal{H}_{N}(\mathcal{P})$ is defined up to multiplication by a $N^{\text {th }}$ root of unity, with no assumption on the weights of $\mathcal{P}$.

Proof By a result of Costantino [15], any change of branching can be achieved by means of a finite sequence of branching transits. The conclusion follows from Corollary 8.6.

Remark 8.3 One can ask if a weakly branched version of Costantino's result holds true: given weakly branched triangulations $(T, \tilde{b})$ and $\left(T, \widetilde{b}^{\prime}\right)$ of $\widehat{V}$ that differ only by the weak branchings, are they related by a sequence of oriented $C$-moves and 
local $b$-transits? The answer is negative. In fact, we know that the state sums $\mathcal{H}_{N}$ have no sign anomaly with respect to $\mathrm{QH}$ transits and oriented $C$-moves, but there are examples of weakly branched triangulations $(T, \tilde{b})$ admitting circuit moves along circuits $\gamma$ such that $h_{c}(\gamma) \neq 0 \in \mathbb{Z} / 2 \mathbb{Z}$.

\section{A Behavior of the sign anomaly under QH transits}

Recall the formula (19). The matrix dilogarithms $\mathcal{R}_{N}$ satisfy Proposition 7.2, whereas the basic dilogarithms $\mathcal{L}_{N}$ satisfy the following relations [4, Proposition 5.3]:

$$
\begin{aligned}
& \mathcal{L}_{N}\left(\Delta, b_{(01)}, w^{\prime}, f^{\prime}, c^{\prime}\right)_{k, j}^{i, l} \equiv \equiv_{N}\left(w_{0}^{\prime}\right)^{\frac{1-N}{2}} T_{k, k^{\prime}} \mathcal{L}_{N}(\Delta, b, w, f, c)_{i^{\prime}, j}^{k^{\prime}, l}\left(T^{-1}\right)^{i^{\prime}, i}, \\
& \mathcal{L}_{N}\left(\Delta, b_{(12)}, w^{\prime}, f^{\prime}, c^{\prime}\right)_{j, l}^{i, k} \equiv_{N}\left(w_{1}^{\prime}\right)^{\frac{N-1}{2}} T_{l, l^{\prime}} \mathcal{L}_{N}(\Delta, b, w, f, c)_{i^{\prime}, l^{\prime}}^{k, l^{\prime}}\left(S^{-1}\right)^{i^{\prime}, i} \\
& \mathcal{L}_{N}\left(\Delta, b_{(23)}, w^{\prime}, f^{\prime}, c^{\prime}\right)_{i, l}^{k, j} \equiv_{N}\left(w_{0}^{\prime}\right)^{\frac{1-N}{2}} S_{l, l^{\prime}} \mathcal{L}_{N}(\Delta, b, w, f, c)_{i, j^{\prime}}^{k, l^{\prime}}\left(S^{-1}\right)^{j^{\prime}, j} .
\end{aligned}
$$

Define the transits of $N^{\text {th }}$ root cross ratio moduli like in (60), by replacing $W(e)$ with the total $N^{\text {th }}$ root modulus $W^{\prime}(e)$ at all edges $e$ of $T$ and $T^{\prime}$. Clearly any QH transit induces a transit of $N^{\text {th }}$ root cross ratio moduli.

Theorem 8.4 [4, Theorem 5.2] The basic matrix dilogarithm identity supported by the Schaeffer $2 \leftrightarrow 3$ transit of $N^{\text {th }}$ root cross ratio moduli holds true up to an $N^{\text {th }}$ root of unity anomaly.

Let us recall the main ideas of the proof. The basic matrix dilogarithm identity supported by the Schaeffer $2 \leftrightarrow 3 \mathrm{QH}$ transit is equivalent to the following equality in $\operatorname{Aut}\left(\mathbb{C}^{N} \otimes \mathbb{C}^{N} \otimes \mathbb{C}^{N}\right)$ :

$$
\Psi_{23}^{1}(V) \Psi_{12}^{3}(U)=\Psi_{12}^{4}(U) \Psi_{13}^{2}(-U V) \Psi_{23}^{0}(V),
$$

where $U$ and $V$ are explicit $N^{2} \times N^{2}$ matrices satisfying $U^{N}=-\mathrm{Id} \otimes \mathrm{Id}, V^{N}=$ $-\mathrm{Id} \otimes \mathrm{Id}$, and $V U=\zeta U V, \zeta$ being a primitive $N^{\text {th }}$ root of 1 , and for a tensor $A$ satisfying $A^{N}=-$ Id (on the appropriate space: $\mathbb{C}^{N}, \mathbb{C}^{N} \otimes \mathbb{C}^{N}$ etc) one sets

$$
\Psi^{i}(A)=\sum_{l=0}^{N-1} A^{l} \prod_{s=1}^{l} \frac{\left(\left(w_{0}^{i}\right)^{\prime}\right)^{-1}\left(\left(w_{1}^{i}\right)^{\prime}\right)^{-1}}{1-\left(\left(w_{0}^{i}\right)^{\prime}\right)^{-1} \zeta^{-s}} .
$$

In (64), $\Psi_{23}^{1}(V)$ means $\Psi^{1}(V)$ acting on the second and third tensorands, ie $\operatorname{Id} \otimes \Psi^{1}(V)$ etc. One can check that $\Psi^{i}$ is uniquely determined up to multiplication by scalars by the functional relation

$$
\Psi^{i}\left(\zeta^{-1} A\right)=\Psi^{i}(A)\left(\frac{1-\left(\left(w_{0}^{i}\right)^{\prime}\right)^{-1}\left(\left(w_{1}^{i}\right)^{\prime}\right)^{-1} A}{\left(\left(w_{0}^{i}\right)^{\prime}\right)^{-1}}\right)=\Psi^{i}(A)\left(\left(w_{0}^{i}\right)^{\prime}-\left(\left(w_{1}^{i}\right)^{\prime}\right)^{-1} A\right)
$$


The identity (64) lifts to the algebra generated by $U$ and $V$, so we have to show

$$
\Psi^{1}(V) \Psi^{3}(U)=\Psi^{4}(U) \Psi^{2}(-U V) \Psi^{0}(V)
$$

when $U^{N}=-1, V^{N}=-1$, and $V U=\zeta U V$. To do it, one uses crucially the relations between $N^{\text {th }}$ root cross ratio moduli implied by the Schaeffer $\mathrm{QH}$ transit. One shows that $U V$ commutes with $P(-U V):=\Psi^{4}(U)^{-1} \Psi^{1}(V) \Psi^{3}(U) \Psi^{0}(V)^{-1}$; hence $P(-U V)$ is a function of $U V$. Moreover, it satisfies

$$
P\left(-\zeta^{-1} U V\right)=P(-U V)\left(\left(w_{0}^{\prime}\right)^{2}-\left(\left(w_{1}^{\prime}\right)^{2}\right)^{-1}(-U V)\right) .
$$

Comparing with (66) we see that $P(-U V)$ and $\Psi^{2}(-U V)$ are equal up to a multiplicative constant $C:=C\left(\left(w_{0}^{\prime}\right)^{2},\left(w_{1}^{\prime}\right)^{2}\right)$. We introduce an $N^{\text {th }}$ root of the reciprocal of $\operatorname{det}\left(\Psi^{i}(A)\right)$ in each entry of $\Psi^{i}(A)$ so that $\operatorname{det}\left(\Psi^{i}(A)\right)=1$ (this is the term $h\left(\left(w_{0}^{i}\right)^{\prime}\right)=g\left(\left(w_{0}^{i}\right)^{\prime}\right) / g(1)$ in $\left.(18)\right)$. Then $\operatorname{det}(P(-U V))=1=C^{N} \operatorname{det}\left(\Psi^{2}(-U V)\right)$ and $C^{N}=1$. As a result the tensor $\mathcal{L}_{N}\left(w_{0}^{\prime}, w_{1}^{\prime}\right)$ can be expressed in terms of $\Psi(A)$ for a suitable $A$; see [4, formulas (32) and (33)].

The basic matrix dilogarithm identities hold true up to an $N^{\text {th }}$ root of unity anomaly only for some $2 \leftrightarrow 3$ branching transits. From (63) one deduces Table 1 of the anomalies produced by the symmetries of the Schaeffer $2 \leftrightarrow 3$ transit (it is enough to consider those induced by the transpositions $(01), \ldots,(34)$ on the vertex ordering used in Figure 17). The transits of $N^{\text {th }}$ root moduli imply that the anomalies are equal for the symmetries induced by (01) and (34), but differ for (12) and (23) because of the occurrence of the opposite exponents $(1-N) / 2,(N-1) / 2$ for the $N^{\text {th }}$ root moduli $\left(w_{0}^{k}\right)^{\prime},\left(w_{1}^{k}\right)^{\prime}$ respectively. For instance $\left(w_{1}^{0}\right)^{\prime}\left(w_{0}^{4}\right)^{\prime}=\left(w_{1}^{1}\right)^{\prime}$, but we see $\left(\left(w_{1}^{0}\right)^{\prime}\right)^{(N-1) / 2}\left(\left(w_{0}^{4}\right)^{\prime}\right)^{(1-N) / 2} \neq\left(\left(w_{1}^{1}\right)^{\prime}\right)^{(N-1) / 2}$.

\begin{tabular}{c|cc|ccc} 
& $\Delta^{1}$ & $\Delta^{3}$ & $\Delta^{0}$ & $\Delta^{2}$ & $\Delta^{4}$ \\
\hline$(01)$ & 1 & $\left(\left(w_{0}^{3}\right)^{\prime}\right)^{\frac{1-N}{2}}$ & 1 & $\left(\left(w_{0}^{2}\right)^{\prime}\right)^{\frac{1-N}{2}}$ & $\left(\left(w_{0}^{4}\right)^{\prime}\right)^{\frac{1-N}{2}}$ \\
$(12)$ & 1 & $\left(\left(w_{1}^{3}\right)^{\prime}\right)^{\frac{N-1}{2}}$ & $\left(\left(w_{0}^{0}\right)^{\prime}\right)^{\frac{1-N}{2}}$ & 1 & $\left(\left(w_{1}^{4}\right)^{\prime}\right)^{\frac{N-1}{2}}$ \\
$(23)$ & $\left(\left(w_{1}^{1}\right)^{\prime}\right)^{\frac{N-1}{2}}$ & 1 & $\left(\left(w_{1}^{0}\right)^{\prime}\right)^{\frac{N-1}{2}}$ & 1 & $\left(\left(w_{0}^{4}\right)^{\prime}\right)^{\frac{1-N}{2}}$ \\
(34) & $\left(\left(w_{0}^{1}\right)^{\prime}\right)^{\frac{1-N}{2}}$ & 1 & $\left(\left(w_{0}^{0}\right)^{\prime}\right)^{\frac{1-N}{2}}$ & $\left(\left(w_{0}^{2}\right)^{\prime}\right)^{\frac{1-N}{2}}$ & 1
\end{tabular}

Table 1

Replacing the tensors $\mathcal{L}_{N}$ with $\mathcal{R}_{N}$ we have:

Proposition 8.5 The matrix dilogarithm identities hold true up to an $N^{\text {th }}$ root of unity anomaly for all QH transits. 
Proof Since we have the identity (64), in the case of the Schaeffer QH transit identity it is enough to compare the symmetrization factors at both sides. They are $\left(\epsilon_{N}:=\right.$ $\left.(-1)^{(N-1) / 2}\right)$ :

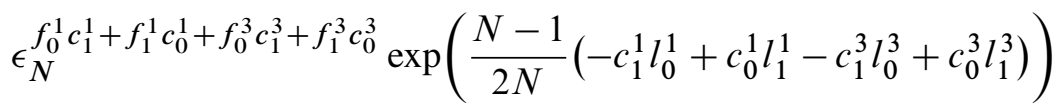

and

$$
\begin{aligned}
\epsilon_{N}^{f_{0}^{0} c_{1}^{0}+f_{1}^{0} c_{0}^{0}+f_{0}^{2} c_{1}^{2}+f_{1}^{2} c_{0}^{2}}+f_{0}^{4} c_{1}^{4}+f_{1}^{4} c_{0}^{4} \\
\quad \times \exp \left(\frac{N-1}{2 N}\left(-c_{1}^{0} l_{0}^{0}+c_{0}^{0} l_{1}^{0}-c_{1}^{2} l_{0}^{2}+c_{0}^{2} l_{1}^{2}-c_{1}^{4} l_{0}^{4}+c_{0}^{4} l_{1}^{4}\right)\right) .
\end{aligned}
$$

A computation shows that the transits of log branches and charges imply that the two exponentials are equal, and with the flattening transit mod 2 that the signs are equal. To prove the claim for all others $2 \leftrightarrow 3 \mathrm{QH}$ transits we compare the anomalies resulting from symmetries on the Schaeffer QH transit. Since all QH transits are branched, the actions by the matrices $S, T$ due to Proposition 7.2 cancel out along interior faces. Table 2 shows the sign anomalies. Again, the transit of charges implies that they are equal at both sides.

\begin{tabular}{c|cc|ccc} 
& $\Delta^{1}$ & $\Delta^{3}$ & $\Delta^{0}$ & $\Delta^{2}$ & $\Delta^{4}$ \\
\hline$(01)$ & 1 & $\epsilon_{N}^{c_{0}^{3}}$ & 1 & $\epsilon_{N}^{c_{0}^{2}}$ & $\epsilon_{N}^{c_{0}^{4}}$ \\
$(12)$ & 1 & $\epsilon_{N}^{c_{1}^{3}}$ & $\epsilon_{N}^{c_{0}^{0}}$ & 1 & $\epsilon_{N}^{c_{1}^{4}}$ \\
$(23)$ & $\epsilon_{N}^{c_{1}^{1}}$ & 1 & $\epsilon_{N}^{c_{1}^{0}}$ & 1 & $\epsilon_{N}^{c_{0}^{4}}$ \\
$(34)$ & $\epsilon_{N}^{c_{0}^{1}}$ & 1 & $\epsilon_{N}^{c_{0}^{0}}$ & $\epsilon_{N}^{c_{0}^{2}}$ & 1
\end{tabular}

Table 2

As usual, the identities for the bubble moves are formal consequence of those for the $2 \leftrightarrow 3$ moves. This concludes the proof.

Corollary 8.6 Let $(T, \tilde{b}, w, f, c) \rightarrow\left(T^{\prime}, \tilde{b}^{\prime}, w^{\prime}, f^{\prime}, c^{\prime}\right)$ be any $Q H$ transit of weakly branched $Q H$ triangulations of a pattern $\mathcal{P}$. Then

$$
\mathcal{H}_{N}(T, \tilde{b}, w, f, c) \equiv_{N} \mathcal{H}_{N}\left(T, \tilde{b}^{\prime}, w^{\prime}, f^{\prime}, c^{\prime}\right) .
$$

Proof If the transit is supported by a MP move it is immediate that $a_{N}(T, \tilde{b})=$ $a_{N}\left(T^{\prime}, \widetilde{b}^{\prime}\right)$, hence the conclusion follows from Proposition 8.5. If it is supported by a 
positive bubble move, spelling the contribution of each term we realize that if

$$
a_{N}(T, \tilde{b})=N^{-v} \phi_{N}^{-q(T, \tilde{b})} c_{N}(T, \tilde{b}), \quad c_{N}(T, \tilde{b})=\epsilon_{N}^{v+l-\frac{1}{2} \sum_{e}\left(n_{+}(e)-n_{-}(e)\right)}
$$

then

$$
\begin{aligned}
& a_{N}\left(T^{\prime}, \tilde{b}^{\prime}\right)=N^{-(v+1)} \phi_{N}^{-q\left(T^{\prime}, \tilde{b}^{\prime}\right)} c_{N}\left(T^{\prime}, \tilde{b}^{\prime}\right), \\
& c_{N}\left(T^{\prime}, \tilde{b}^{\prime}\right)=\epsilon_{N}^{(v+1)+(l+3)-\left(2+\frac{1}{2} \sum_{e}\left(n_{+}(e)-n_{-}(e)\right),\right.}
\end{aligned}
$$

and $\phi_{N}^{-q\left(T^{\prime}, \tilde{b}^{\prime}\right)}=\phi_{N}^{-q(T, \tilde{b})}$. Hence $a_{N}\left(T^{\prime}, \tilde{b}^{\prime}\right)=N^{-1} a_{N}(T, \tilde{b})$, as it must be in order to ensure the invariance under the bubble move, which increases by 1 the number of internal vertices.

\section{B Behavior of the sign anomaly under a change of weak branching}

A change of weak branching $\tilde{b} \rightarrow \widetilde{b}^{\prime}$ modifies $a_{N}(T, \tilde{b})$ by its subfactors $\phi_{N}^{-q(T, \tilde{b})}$ and

$$
c_{N}^{*}(T, \tilde{b}):=\epsilon_{N}^{l-\frac{1}{2} \sum_{e}\left(n_{+}(e)-n_{-}(e)\right)} .
$$

By the proof of Proposition 7.1, $\phi_{N}^{-q(T, \tilde{b})}$ and the unnormalized QH state sum have reciprocal variations. So it remains to analyze the behavior of $c_{N}^{*}$ with respect to oriented $C$-moves and the circuit move of Lemma 6.4.

It is relevant here to clarify the nature of $c_{N}^{*}$. By extending [8, Chapter 7], the paper [9] provides a combinatorial realization of spin structures on 3-manifolds, based on weakly branched triangulations instead of branched ones. In particular, one associates to every weakly branched triangulation $(T, \tilde{b})$ a framing $\nu \tilde{b}$ of the manifold defined along $\operatorname{Sing}(P)$, and computes a cellular cochain $\alpha \widetilde{b} \in C^{2}(P ; \mathbb{Z} / 2 \mathbb{Z})$ representing the obstruction to extend $v \widetilde{b}$ over the whole of the spine $P$. Consider the cellular 2-chain $R(P)=\sum_{R} R \in C_{2}(P ; \mathbb{Z} / 2 \mathbb{Z})$, where $R$ varies among the 2 -regions of $P$. It is a fact that

$$
l-\frac{1}{2} \sum_{e}\left(n_{+}(e)-n_{-}(e)\right)=\alpha \tilde{b}(R(P)) \bmod 2 .
$$

How $\alpha \tilde{b}$, whence $\alpha \tilde{b}(R(P))$, varies with the weak branching is part of the theory developed in [9]. As a consequence we get:

Lemma 8.7 For any change of weak branching $\widetilde{b} \rightarrow \widetilde{b}^{\prime}$ preserving the induced prebranching we have

$$
\mathcal{H}_{N}(T, \tilde{b}, w, f, c) \equiv_{N} \mathcal{H}_{N}\left(T, \tilde{b}^{\prime}, w, f, c\right) .
$$


Proof It is enough to show the result for one generating oriented $C$-move $\sigma=(0123)$. By local inspection one checks that $\alpha_{\widetilde{b}^{\prime}}(R(P))=\alpha \widetilde{b}(R(P))+1$, so $c_{N}^{*}\left(T, \tilde{b}^{\prime}\right)=$ $\epsilon_{N} c_{N}^{*}(T, \tilde{b})$. On the other hand, using Proposition 7.2, the factorization $(0123)=$ $(23) \cdot(12) \cdot(01)$, and the relation $c_{0}+c_{1}+c_{2}=1$, we see that $\sigma$ changes the unnormalized QH state sum by $\epsilon_{N}^{c_{1}} \epsilon_{N}^{c_{2}} \epsilon_{N}^{c_{0}}=\epsilon_{N}$. Hence the two sign variations are equal.

Lemma 8.8 For any change of weak branching $\widetilde{b} \rightarrow \widetilde{b}^{\prime}$ induced by one circuit move along a circuit $\gamma$ we have

$$
\mathcal{H}_{N}(T, \tilde{b}, w, f, c) \equiv_{N} \epsilon_{N}^{h_{c}(\gamma)} \mathcal{H}_{N}\left(T, \tilde{b}^{\prime}, w, f, c\right),
$$

where $h_{c}$ is the bulk $c$-weight of $\mathcal{P}$. In particular, $\mathcal{H}_{N}(\mathcal{P})$ has no sign anomaly if $h_{c}=0$.

Proof Let us realize the circuit move in terms of $\mathcal{N}$-graphs. By the third identity of Proposition 7.2 the move changes the state sum by one factor $\epsilon_{N}^{c_{0}}$ for each crossing of $\gamma$. It is easy to check that their product computes $h_{c}(\gamma)$. On the other hand $\alpha_{\tilde{b}^{\prime}}(R(P))=\alpha \widetilde{b}(R(P))$, so that $c_{N}^{*}\left(T, \widetilde{b}^{\prime}\right)=c_{N}^{*}(T, \tilde{b})$.

\section{Examples}

In this section we describe the quantum hyperbolic invariants of the figure-eight knot complement, its sister, and the complement of the knot $5_{2}$.

We give also some samples of numerical computations obtained by using Maple, which are part of our current analytical and numerical exploration of the QHI. At first we quote that they corroborate the invariance of the $\mathrm{QH}$ state sums with respect to the choice of flattenings or charges having the same weights. In the case of the sister manifold, they confirm also the invariance of the QH state sums supported by weakly branched triangulations. Moreover, the numerical computations that we present below are proving:

- The actual dependence of the invariants on the weights (and not only on the characters $\rho$ ).

- This dependence persists as $N \rightarrow \infty$.

- For some weights the invariants seem to grow exponentially with $N$, and yield instances of the volume conjecture, and for some other weights they do not. 
Other interesting phenomena happen, that deserve to be understood. For instance, in the case of the complements of the knots $4_{1}$ and $5_{2}$, it appears that the asymptotic behavior of the absolute values of the invariants do not depend on the values on the meridian of the charge weights or the flattening weights.

\section{A Notations}

Let $(\Delta, b, w, f, c)$ be a quantum 3 -simplex. Put $d_{k}:=f_{k}-*_{j} c_{k}, k \in \mathbb{Z} / 3 \mathbb{Z}$. We call $d_{k}$ an edge color. In order to simplify the formulas we denote the $N^{\text {th }}$ roots of the cross ratios $w_{k}$ by bold letters $\boldsymbol{w}_{k}$ (rather than $w_{k}^{\prime}$, as we did in (14)), and call them q-shape parameters. So

$$
\boldsymbol{w}_{k}:=\exp \left(\frac{1}{N}\left(\log \left(w_{k}\right)+\pi i(N+1) d_{k}\right)\right)
$$

For any cusped manifold $M$ and any $\mathrm{QH}$ triangulation $\mathcal{T}$ of $M$, we have the tetrahedral and edge relations (see (15), (22) and (25))

$$
\sum_{k=0,1,2}\left(\log \left(w_{k}\right)+\pi i d_{k}\right)=-*_{b} \pi i, \quad \sum_{E \rightarrow e} *_{E}(\log (w(E))+\pi i d(E))=-2 \pi i,
$$

and

$$
\prod_{k=0,1,2} \boldsymbol{w}_{k}=e^{-*_{b} \pi i / N}, \quad \prod_{E \rightarrow e} \boldsymbol{w}(E)^{*_{E}}=e^{-2 \pi i / N},
$$

where, as usual, $d(E)=d_{k}$ and $\boldsymbol{w}(E)=\boldsymbol{w}_{k}$ if $E$ is the edge $E_{k}$ or the opposite edge of a quantum 3-simplex $(\Delta, b, w, f, c)$ of $\mathcal{T}$, and $*_{E}:=*_{b}$. We denote by $G_{N}(T, \widetilde{b})$ the variety thus defined by the q-shape parameters, and call it the $Q H$ gluing variety (NB: it coincides with the variety $G(T, \widetilde{b}, c)_{N}$ of Section $3 \mathrm{~B}$; to simplify notations, here we drop the reference to the charge $c$ ).

As usual, denote by $V$ a compact 3-manifold with one torus boundary component such that $M$ is diffeomorphic to the interior of $V$. We compute the invariant $\mathcal{H}_{N}\left(V, \rho, k_{f}, k_{c}, h_{f}, h_{c}\right)$ by means of the function $\mathcal{H}_{N}^{\prime}(T, \tilde{b}, c)$ on $G_{N}(T, \tilde{b})$ introduced after Definition 3.5. As $\mathcal{H}_{N}^{\prime}(T, \tilde{b}, c)$ is formulated in terms of q-shape parameters and charges, we express equivalently $\mathcal{H}_{N}\left(V, \rho, k_{f}, k_{c}, h_{f}, h_{c}\right)$ as $\mathcal{H}_{N}\left(V, \rho, \kappa, k_{c}, h, h_{c}\right)$, using the classes $\kappa$ and $h$ obtained from the flattening weights $k_{f}$ and $h_{f}$ by replacing the flattenings $f_{k}$ by the edge colors $d_{k}$ in the respective formulas (see Definition 4.10, and (26) for $\kappa$ ). Clearly $\kappa=k_{f}-\pi i k_{c}$ and $h=h_{c}+h_{f}$. We call $\kappa$ and $h$ the reduced boundary and bulk weights. By (1) and (28) one has the compatibility relations

$$
\kappa(a)=d_{w}(a) \quad \bmod i \pi \mathbb{Z}, \quad\left(\kappa(a)-d_{w}(a)\right) / i \pi=\iota^{*}(h)(a) \bmod 2 \mathbb{Z}
$$

for all $a \in H_{1}(\partial V ; \mathbb{Z})$. 
We consider triangulations with ordered tetrahedra. We denote by $u, v, w \ldots$ (resp. $\boldsymbol{u}, \boldsymbol{v}, \boldsymbol{w} \ldots$, resp. $a, b, c \ldots$, resp. $a^{\prime}, b^{\prime}, c^{\prime} \ldots$ ) the triples of cross ratios (resp. q-shape parameters, resp. edge colors, resp. charges) of the tetrahedra, in the same order.

\section{B The figure-eight knot complement}

The Epstein-Penner decomposition of $M$ is an ideal triangulation $T$ having a branching $b$ and two tetrahedra, with opposite $b$-orientations. We showed the dual spine in Figure 6. We denote by $\Delta^{0}$ the tetrahedron with positive $b$-orientation. In [5] we described the flattenings at positive points of the gluing variety $G(T, b)$, as well as the charges and the $\mathrm{QH}$ state sums carried by $(T, b)$.

9B1 The QH gluing variety The gluing variety $G(T, b)$ is the irreducible plane curve with coordinates $\left(u_{0}, v_{0}\right)$ and defining equation $u_{1} u_{2}^{2} v_{0}^{-2} v_{1}^{-1}=1$, which may be written as $u_{0}^{2} v_{0}^{2}=\left(1-u_{0}\right)\left(1-v_{0}\right)$ (as usual $u_{i+1}=1 /\left(1-u_{i}\right)$ and similarly for the $\left.v_{i}\right)$. The point $\left(e^{\pi i / 3}, e^{-\pi i / 3}\right)$ realizes the complete hyperbolic structure. Let $l$ and $m$ be the canonical longitude and the meridian of the figure-eight knot, and let $\left(u_{0}, v_{0}\right) \in G(T, b)$. The dilation factors of the holonomy $\rho:=\rho\left(u_{0}, v_{0}\right)$ are given by

$$
\operatorname{hol}_{l}(\rho)=u_{0}^{2} u_{2}^{-2}=\frac{u_{0}^{4}}{\left(1-u_{0}\right)^{2}}, \quad \operatorname{hol}_{m}(\rho)=u_{2} v_{2}=\frac{\left(u_{0}-1\right)\left(v_{0}-1\right)}{u_{0} v_{0}}
$$

The tetrahedral and edge relations between q-shape parameters are

$$
\begin{aligned}
\boldsymbol{u}_{0} \boldsymbol{u}_{1} \boldsymbol{u}_{2} & =e^{-\pi i / N}, & \boldsymbol{v}_{0} \boldsymbol{v}_{1} \boldsymbol{v}_{2} & =e^{+\pi i / N}, \\
\boldsymbol{u}_{1} \boldsymbol{u}_{2}^{2} \boldsymbol{v}_{0}^{-2} \boldsymbol{v}_{1}^{-1} & =e^{-2 \pi i / N}, & \boldsymbol{u}_{1} \boldsymbol{u}_{0}^{2} \boldsymbol{v}_{2}^{-2} \boldsymbol{v}_{1}^{-1} & =e^{-2 \pi i / N} .
\end{aligned}
$$

Together with the identities $\left(\boldsymbol{u}_{i+1}\right)^{N}=1 /\left(1-\left(\boldsymbol{u}_{i}\right)^{N}\right)$ and $\left(\boldsymbol{v}_{i+1}\right)^{N}=1 /\left(1-\left(\boldsymbol{v}_{i}\right)^{N}\right)$, these relations define $G_{N}(T, b)$. Using the tetrahedral relations it is easy to see that the two edge relations are equivalent. Recall the reduced weights $h$ and $\kappa$ introduced in Section 9A. By (69) we have

$$
\boldsymbol{u}_{0}^{2} \boldsymbol{u}_{2}^{-2}=e^{\kappa(l) / N+\pi i h(l)}, \quad \boldsymbol{u}_{2} \boldsymbol{v}_{2}=e^{\kappa(m) / N+\pi i h(m)} .
$$

As $M$ is a knot complement, the bulk weight $h$ is determined by $\kappa$, and similarly $h_{c}$ is determined by $k_{c}$. Moreover, $h(l)=0$, so $h$ is nonzero if and only if $h(m)=$ $a_{2}+b_{2}=1 \bmod 2$.

Over the point $\left(e^{\pi i / 3}, e^{-\pi i / 3}\right)$ we have $\kappa(m) \in \pi i \mathbb{Z}$ and $\kappa(l) \in 2 \pi i \mathbb{Z}$, and the tetrahedral relations, the edge relations, and the compatibility relations with $\kappa$ imply the following expressions of the edge colors $a_{k}$ and $b_{k}$ in terms of $a_{0}$ and $\kappa: a_{2}=$ 
$-2-a_{0}-a_{1}, b_{2}=2-b_{0}-b_{1}$, and

(71) $a_{1}=-2 a_{0}+\frac{\kappa(l)}{2 \pi i}-2, \quad b_{0}=\frac{\kappa(m)}{\pi i}-a_{0}, \quad b_{1}=2 a_{0}-\frac{2 \kappa(m)}{\pi i}-\frac{\kappa(l)}{2 \pi i}+2$.

For points $(\boldsymbol{u}, \boldsymbol{v}) \in G_{N}(T, b)$ lying over a sufficiently small neighborhood of the point $\left(e^{\pi i / 3}, e^{-\pi i / 3}\right) \in G(T, b)$, the same expressions are valid if one replaces $\kappa(\cdot) / \pi i$ with $\left(\kappa(\cdot)-\log \left(\right.\right.$ hol $\left.\left._{\bullet}(\rho)\right)\right) / \pi i$.

Similarly the charges $a_{k}^{\prime}$ and $b_{k}^{\prime}$ are given by

(72) $a_{1}^{\prime}=-2 a_{0}^{\prime}+\frac{k_{c}(l)}{2}+1, \quad b_{0}^{\prime}=a_{0}^{\prime}-k_{c}(m), \quad b_{1}^{\prime}=-2 a_{0}^{\prime}+2 k_{c}(m)+\frac{k_{c}(l)}{2}+1$.

Let $M^{\prime}$ be a closed hyperbolic $(p, q)$-Dehn filling of $M$ represented by $\left(u_{0}, v_{0}\right) \in$ $G(T, b)$ in a small neighborhood of $\left(e^{\pi i / 3}, e^{-\pi i / 3}\right)$. The holonomy $\rho$ factors through the quotient map $\pi_{1}(M) \rightarrow \pi_{1}\left(M^{\prime}\right)$ to define the holonomy $\rho^{\prime}$ of $M^{\prime}$. One has

$$
p \log \left(\operatorname{hol}_{m}\left(\rho^{\prime}\right)\right)+q \log \left(\operatorname{hol}_{l}\left(\rho^{\prime}\right)\right)=2 \pi i,
$$

and letting $r, s \in \mathbb{Z}$ be such that $p s-q r=1$, the reduced weight $\kappa$ satisfies $\kappa\left(m^{p} l^{q}\right)=$ 0 if and only if

$$
a_{1}=r-2-2 a_{0}, \quad b_{0}=-2 s-a_{0}, \quad b_{1}=2-r+4 s+2 a_{0} .
$$

Similar formulas express the identity $k_{c}\left(m^{p} l^{q}\right)=0$.

9B2 The state sum formulas Recall the notations of Section 3A2. For any $(\boldsymbol{u}, \boldsymbol{v}) \in$ $G_{N}(T, b)$ and any charge $c$ on $T$, the $\mathrm{QH}$ state sum

$$
\begin{aligned}
& \mathcal{H}_{N}^{\prime}(T, b, c)(\boldsymbol{u}, \boldsymbol{v}) \\
& \quad=\left(\boldsymbol{u}_{0}^{-a_{1}^{\prime}} \boldsymbol{u}_{1}^{a_{0}^{\prime}} \boldsymbol{v}_{0}^{-b_{1}^{\prime}} \boldsymbol{v}_{1}^{b_{0}^{\prime}}\right)^{\frac{N-1}{2}} \frac{\left[\boldsymbol{v}_{0}\right] g\left(\boldsymbol{u}_{0}\right)}{g\left(\boldsymbol{v}_{0}\right)} \sum_{\alpha, \beta=0}^{N-1} \zeta^{\beta^{2}-\alpha^{2}} \frac{\omega\left(\boldsymbol{u}_{0}, \boldsymbol{u}_{1}^{-1} \mid N-\beta\right)}{\omega\left(\boldsymbol{v}_{0} / \zeta, \boldsymbol{v}_{1}^{-1} \mid N-\alpha\right)}
\end{aligned}
$$

computes the invariant $\mathcal{H}_{N}\left(V, \rho, \kappa, k_{c}, h, h_{c}\right)$, and defines a (nonconstant) rational function on $G_{N}(T, b)$. By a surgery theorem proved in [5], at points $(\boldsymbol{u}, \boldsymbol{v})$ where $\kappa$ and $k_{c}$ satisfy $\kappa\left(m^{p} l^{q}\right)=k_{c}\left(m^{p} l^{q}\right)=0$ as above, $\mathcal{H}_{N}\left(V, \rho, \kappa, k_{c}, h, h_{c}\right)$ coincides with the $N^{\text {th }}$ quantum hyperbolic invariant of the closed manifold $\left(M^{\prime}, L, \rho^{\prime}\right)$, where $L$ is the surgery core.

$9 B 3$ Some numerical results Denote by $\rho(A, B):=\rho\left(u_{0}(A, B), v_{0}(A, B)\right)$ the holonomy of $M$ given by the shape parameter $u_{0}(A, B):=e^{\pi i / 3}+A+i B$ and the solution $v_{0}(A, B)$ of the gluing equation $u_{0}(A, B)^{2} v_{0}(A, B)^{2}-\left(1-u_{0}(A, B)\right)(1-$ $\left.v_{0}(A, B)\right)=0$ such that $u_{0}(A, B)$ has positive imaginary part and $v_{0}(A, B)$ has negative imaginary part. So $\rho(0,0)$ is the hyperbolic holonomy. 
Recall that $h$ and $h_{c}$ are determined by $\kappa$ and $k_{c}$ respectively. In the following table we present some values of $\left|\mathcal{H}_{15}\left(V, \rho(A, B), \kappa, k_{c}, h, h_{c}\right)\right|$ up to 4 digits, where $(A, B)$ and $k_{c}(l)$ are as indicated on the top and the left of the corresponding column and row $\left((A, B)_{(2,3)}:=(0.06734378 \ldots,-.42400885 \ldots)\right.$ defines the holonomy of the $(2,3)-$ Dehn filling of $M)$, and we put $\kappa(m)=k_{c}(m)=0$ and $\kappa(l)=k_{f}(l)-\pi i k_{c}(l)$, where $k_{f}(l)=L([w ; f])(l)$ has the collection of shape parameters $w:=w(A, B)$ determined by $\left(u_{0}(A, B), v_{0}(A, B)\right)$, and a (compatible) flattening $f$ such that $L([w(0,0) ; f])=$ $-2 \pi i$ at the complete hyperbolic structure.

\begin{tabular}{r|rrrrr} 
& $(0,0)$ & $(1,0)$ & $(1,0.5)$ & \multicolumn{1}{c}{$(1,1)$} & $(A, B)_{(2,3)}$ \\
\hline 6 & 2.5587 & 2.6504 & 2.0018 & 1.6118 & 5.0307 \\
4 & 58.5466 & 58.3761 & 47.0533 & 39.9892 & 95.0326 \\
2 & 2.1356 & 2.0279 & 1.9058 & 1.8138 & 2.7491 \\
0 & 77.4851 & 77.5401 & 77.5885 & 77.6997 & 76.4850 \\
-2 & 0.1118 & 0.1620 & 0.1672 & 0.1746 & 0.0650 \\
-4 & 77.4851 & 77.5401 & 95.8738 & 112.7032 & 47.4247 \\
-6 & 2.1356 & 2.0279 & 2.7647 & 3.5183 & 1.0549 \\
-8 & 58.5466 & 58.3761 & 89.7752 & 124.5239 & 21.8881 \\
-10 & 2.5587 & 2.6504 & 4.2536 & 6.1199 & 0.7577 \\
-12 & 33.2019 & 33.1224 & 63.6250 & 104.4917 & 7.4274
\end{tabular}

Table 3: Entries accurate to four decimal places

Note that the table shows that the invariants depend on the character and the weights. Also, the first two columns show a symmetry about $k_{c}(l)=-2$ (that is, when $(A, B)=$ $(0,0)$, about $\kappa(l)=0)$, corresponding to a change of orientation of $l$. The dominant rows are for $k_{c}(l)=0$ or -4 (that is, when $(A, B)=(0,0)$, about $\kappa(l)=-2 \pi i$ or $2 \pi i)$.

Now consider the behavior of $\left|\mathcal{H}_{N}\left(V, \rho(A, B), \kappa, k_{c}, h, h_{c}\right)\right|$ as $N \rightarrow+\infty$. Put

$$
G_{N}(\kappa(l)):=\pi \log \left(\left|\mathcal{H}_{N+2}\left(V, \rho(0,0), \kappa, k_{c}, h, h_{c}\right)\right| /\left|\mathcal{H}_{N}\left(V, \rho(0,0), \kappa, k_{c}, h, h_{c}\right)\right|\right),
$$

where the arguments are as above except that we fix $(A, B)=(0,0)$ (the complete hyperbolic structure). Let $\mathcal{H}_{N}^{\prime \prime}(T, b, c)=\mathcal{H}_{N}^{\prime}(T, b, c) /\left(\boldsymbol{u}_{0}^{-a_{1}^{\prime}} \boldsymbol{u}_{1}^{a_{0}^{\prime}} \boldsymbol{v}_{0}^{-b_{1}^{\prime}} \boldsymbol{v}_{1}^{b_{0}^{\prime}}\right)^{(N-1) / 2}$, where the denominator is the product of the matrix dilogarithm symmetrization factors. Note that

$$
G_{N}:=\pi \log \left(\left|\mathcal{H}_{N+2}^{\prime \prime}(T, b, c)(\boldsymbol{u}, \boldsymbol{v})\right| /\left|\mathcal{H}_{N}^{\prime \prime}(T, b, c)(\boldsymbol{u}, \boldsymbol{v})\right|\right)
$$

and $G_{N}(\kappa(l))$ are equivalent as $N \rightarrow+\infty$. The following table gives a sample of values of $G_{151}$, where $\kappa(l)$ takes the values indicated in the first row. 


\begin{tabular}{c|ccccc} 
& $-6 \pi i$ & $-4 \pi i$ & $-2 \pi i$ & 0 & $2 \pi i$ \\
\hline$G_{151}$ & 2.03069 & -0.49036 & 2.02968 & 0.48922 & 2.02968
\end{tabular}

Table 4: Entries accurate to five decimal places

Note the dependence on the weights persists as $N \gg 1$. Further numerical computations show that $G_{N}( \pm 2 \pi i)$ converges to $\operatorname{Vol}(M) \approx 2.02988321 \ldots$ as $N \gg 1$.

\section{C The complement of the knot $5_{2}$}

The ideal triangulation $T$ of $M$ with smallest complexity has three tetrahedra $\Delta^{0}$, $\Delta^{1}, \Delta^{2}$, and it has a branching $b$ that gives each tetrahedron the negative branching orientation. This triangulation is the one provided by SnapPea; we keep the same ordering of tetrahedra. The branching $b$ is determined by the ordering it induces on the set of vertices of $\Delta^{0}$, which is obtained from that provided by SnapPea by applying the permutation $\left(v_{0}, v_{1}, v_{2}, v_{3}\right) \mapsto\left(v_{1}, v_{2}, v_{0}, v_{3}\right)$.

9C1 The QH gluing variety The edge relations of $(T, b)$ are $u_{0} u_{2} v_{0}^{2} w_{1} w_{2}=1$, $u_{1} u_{2} v_{2} v_{1}^{2} w_{0} w_{1}=1$, and $u_{0} u_{1} v_{2} w_{0} w_{2}=1$. By the tetrahedral relations they reduce to two independent relations, so the gluing variety of $(T, b)$ is the irreducible curve in $\mathbb{C}_{*}^{3}$ with coordinates $\left(u_{0}, v_{0}, w_{0}\right)$ and defining equations

$$
u_{1} v_{0}^{-2} w_{0}=1, \quad u_{2} v_{2}^{-1} w_{1}=1 .
$$

The complete hyperbolic structure is realized by the point $\left(u_{\text {hyp }}, v_{\text {hyp }}, w_{\text {hyp }}\right)$ given up to 8 digits by (the imaginary parts are negative since all tetrahedra have the negative branching orientation)

$$
\begin{aligned}
u_{\text {hyp }} & \approx 0.21507987-i 1.30714121, \\
v_{\text {hyp }} & \approx 0.33764102-i 0.56227951, \\
w_{\text {hyp }} & \approx 0.33764102-i 0.56227951 .
\end{aligned}
$$

Since $G(T, b)$ is irreducible and $\left(u_{\text {hyp }}^{-1}, v_{\text {hyp }}^{-1}, w_{\text {hyp }}^{-1}\right)$ is a positive point, it is a rich variety. Let $l$ and $m$ be the canonical longitude and the meridian of the knot, and let $\left(u_{0}, v_{0}, w_{0}\right) \in G(T, b)$. The dilation factors of the holonomy $\rho:=\rho\left(u_{0}, v_{0}, w_{0}\right)$ are given by

$$
\begin{aligned}
& \operatorname{hol}_{l}(\rho)=u_{1}^{2} u_{2}^{-3} v_{2} v_{0}^{-2} w_{1}=\frac{u_{0}^{3}\left(v_{0}-1\right)}{\left(1-u_{0}\right)^{2}\left(u_{0}-1\right)^{3} v_{0}^{3}\left(1-w_{0}\right)} \\
& \operatorname{hol}_{m}(\rho)=u_{1} v_{0}^{-1}=\frac{1}{\left(1-u_{0}\right) v_{0}}
\end{aligned}
$$


The tetrahedral and edges relations between q-shape parameters are (note that the exponents -1 on the left of the edge relations are due to the negative branching orientation of the tetrahedra)

$$
\begin{aligned}
\boldsymbol{u}_{0} \boldsymbol{u}_{1} \boldsymbol{u}_{2} & =e^{+\pi i / N}, \\
\boldsymbol{v}_{0} \boldsymbol{v}_{1} \boldsymbol{v}_{2} & =e^{+\pi i / N}, \quad\left(\boldsymbol{u}_{0} \boldsymbol{u}_{2} \boldsymbol{v}_{0}^{2} \boldsymbol{w}_{1} \boldsymbol{w}_{2}\right)^{-1}=e^{-2 \pi i / N}, \\
\boldsymbol{w}_{0} \boldsymbol{w}_{1} \boldsymbol{w}_{2} & =e^{+\pi i / N}, \quad\left(\boldsymbol{u}_{1} \boldsymbol{u}_{2} \boldsymbol{v}_{1}^{2} \boldsymbol{w}_{0} \boldsymbol{w}_{1}\right)^{-1}=e^{-2 \pi i / N}, \\
\left(\boldsymbol{u}_{0} \boldsymbol{u}_{1} \boldsymbol{v}_{2} \boldsymbol{w}_{0} \boldsymbol{w}_{2}\right)^{-1} & =e^{-2 \pi i / N} .
\end{aligned}
$$

Together with the identity $\left(\boldsymbol{u}_{i+1}\right)^{N}=1 /\left(1-\left(\boldsymbol{u}_{i}\right)^{N}\right)$ and the similar ones for the $\left(\boldsymbol{v}_{i}\right)^{N}$ and the $\left(\boldsymbol{w}_{i}\right)^{N}$, these relations define $G_{N}(T, b)$. Using the tetrahedral relations it is easy to see that any of the edge relations is a consequence of the other two. By definition, the weights satisfy

$$
\boldsymbol{u}_{1}^{2} \boldsymbol{u}_{2}^{-3} \boldsymbol{v}_{2} \boldsymbol{v}_{0}^{-2} \boldsymbol{w}_{1}=e^{k(l) / N+\pi i h(l)}, \quad \boldsymbol{u}_{1} \boldsymbol{v}_{0}^{-1}=e^{k(m) / N+\pi i h(m)} .
$$

As $M$ is a knot complement, the bulk weight $h$ is determined by $\kappa$, and similarly $h_{c}$ is determined by $k_{c}$. Moreover $h(l)=0$, so $h$ is nonzero if and only if $h(m)=a_{1}+b_{0}=1$ $\bmod 2$.

At the complete hyperbolic structure we have $\kappa(m) \in \pi i \mathbb{Z}$ and $\kappa(l) \in 2 \pi i \mathbb{Z}$, and the tetrahedral relations, the edge relations, and the compatibility relations with $\kappa$ imply the following expressions of the edge colors $a_{k}, b_{k}$ and $c_{k}$, in terms of $a_{0}, a_{1}$ and $\kappa$ :

$$
\begin{array}{lll}
b_{0}=a_{1}-\frac{\kappa(m)}{\pi i}, & a_{2}=2-a_{0}-a_{1}, \\
c_{0}=-4+a_{1}-2 \frac{\kappa(m)}{\pi i}, & c_{1}=-a_{0}-a_{1}-\frac{\kappa(m)}{\pi i}+\frac{\kappa(l)}{2 \pi i}, & c_{2}=2-c_{0}-c_{1} .
\end{array}
$$

If $(\boldsymbol{u}, \boldsymbol{v}, \boldsymbol{w}) \in G_{N}(T, b)$ lies over a point in a small neighborhood of ( $\left.u_{\text {hyp }}, v_{\text {hyp }}, w_{\text {hyp }}\right) \in$ $G(T, b)$, then the same expressions are valid if one replaces $\kappa(\cdot) / \pi i$ with $(\kappa(\cdot)-$ $\log (\operatorname{hol} \bullet(\rho))) / \pi i$.

Similarly, the charges $a_{k}^{\prime}, b_{k}^{\prime}$ and $c_{k}^{\prime}$ are given by

$$
\begin{array}{ll}
b_{0}^{\prime}=a_{1}^{\prime}-k_{c}(m), & b_{1}^{\prime}=-1+2 a_{0}^{\prime}+a_{1}^{\prime}+2 k_{c}(m)-k_{c}(l) / 2, \\
c_{0}^{\prime}=a_{1}^{\prime}-2 k_{c}(m), & c_{1}^{\prime}=1-a_{0}^{\prime}-a_{1}^{\prime}-k_{c}(m)+k_{c}(l) / 2 .
\end{array}
$$

9C2 The state sum formulas For any $(\boldsymbol{u}, \boldsymbol{v}, \boldsymbol{w}) \in G(T, b)$ and any charge $c$ on $T$ we have (here we denote by $\mathcal{R}_{N}(\Delta, \boldsymbol{w})$ the tensor $\mathcal{R}_{N, *_{b}, c}\left(\boldsymbol{w}_{0}, \boldsymbol{w}_{1}\right)$ ) 


$$
\begin{aligned}
& \mathcal{H}_{N}^{\prime}(T, b, c)(\boldsymbol{u}, \boldsymbol{v}, \boldsymbol{w}) \\
&= \sum_{a, \ldots, f=0}^{N-1} \mathcal{R}_{N}\left(\Delta^{0}, \boldsymbol{u}\right)_{b, c}^{d, a} \mathcal{R}_{N}\left(\Delta^{1}, \boldsymbol{v}\right)_{e, a}^{f, b} \mathcal{R}_{N}\left(\Delta^{2}, \boldsymbol{w}\right)_{d, f}^{c, e} \\
&=\left(\boldsymbol{u}_{0}^{-a_{1}^{\prime}} \boldsymbol{u}_{1}^{a_{0}^{\prime}} \boldsymbol{v}_{0}^{-b_{1}^{\prime}} \boldsymbol{v}_{1}^{b_{0}^{\prime}} \boldsymbol{w}_{0}^{-c_{1}^{\prime}} \boldsymbol{w}_{1}^{c_{0}^{\prime}}\right)^{(N-1) / 2} \frac{\left[\boldsymbol{u}_{0}\right]\left[\boldsymbol{v}_{0}\right]\left[\boldsymbol{w}_{0}\right] g(1)^{3}}{g\left(\boldsymbol{u}_{0}\right) g\left(\boldsymbol{v}_{0}\right) g\left(\boldsymbol{w}_{0}\right)} \\
& \quad \times \sum_{b, c, d=0}^{N-1} \frac{\zeta^{(b+c-d)(c+d)}}{\omega\left(\boldsymbol{u}_{0} / \zeta, \boldsymbol{u}_{1}^{-1} \mid b-d\right) \omega\left(\boldsymbol{v}_{0} / \zeta, \boldsymbol{v}_{1}^{-1} \mid d\right) \omega\left(\boldsymbol{w}_{0} / \zeta, \boldsymbol{w}_{1}^{-1} \mid d-c\right)}
\end{aligned}
$$

The QH state sum $\mathcal{H}_{N}^{\prime}(T, b, c)(\boldsymbol{u}, \boldsymbol{v}, \boldsymbol{w})$ computes the invariant $\mathcal{H}_{N}\left(V, \rho, \kappa, k_{c}, h, h_{c}\right)$ and defines a (nonconstant) rational function on $G_{N}(T, b)$. As for the figure-eight knot complement, and using the same notations, at a point $(\boldsymbol{u}, \boldsymbol{v}, \boldsymbol{w})$ such that $\kappa\left(m^{p} l^{q}\right)=$ $k_{c}\left(m^{p} l^{q}\right)=0, \mathcal{H}_{N}\left(V, \rho, \kappa, k_{c}, h, h_{c}\right)$ coincides with the $N^{\text {th }}$ quantum hyperbolic invariant of the triple $\left(M^{\prime}, L, \rho^{\prime}\right)$ obtained by hyperbolic $(p, q)$-Dehn filling of $M$.

9C3 Some numerical results Keeping the same notations as in Section 9B3, we present below some values of $\left|\mathcal{H}_{15}\left(V, \rho(A, B), \kappa, k_{c}, h, h_{c}\right)\right|$ up to 4 digits, and some values of $G_{121}$. For each of the shape parameter $u_{0}(A, B):=u_{\text {hyp }}+A+$ $i B$ that we consider, with the exception of $u_{0}(0,0)$, there are two distinct points $\left(u_{0}(A, B), v_{0}(A, B), w_{0}(A, B)\right)_{ \pm} \in G(T, b)$ such that both $u_{0}(A, B), v_{0}(A, B)$ and $w_{0}(A, B)$ have negative imaginary parts. We take again $\kappa(m)=k_{c}(m)=0$, but now $k_{c}(l)=0$ and hence $\kappa(l)=k_{f}(l)=L([w ; f])(l)$, where $w:=w_{ \pm}(A, B)$ is determined by $\left(u_{0}(A, B), v_{0}(A, B), w_{0}(A, B)\right)_{ \pm}$, and $f$ is a (compatible) flattening such that, at the complete hyperbolic structure, $k_{f}^{0}:=L([w(0,0) ; f])$ takes the value indicated on the left of each row.

\begin{tabular}{c|rrrrr} 
& \multicolumn{1}{|c}{$(0,0)$} & $(-0.5,0.5)_{+}$ & $(0.5,0.2)_{+}$ & $(0.7,0.3)_{+}$ & $(0.7,0.3)_{-}$ \\
\hline$-6 \pi i$ & 428.2809 & 437.3709 & 474.4262 & 447.6316 & 488.3855 \\
$-4 \pi i$ & 55.2674 & 70.5945 & 65.7471 & 90.4874 & 68.6357 \\
$-2 \pi i$ & 470.6170 & 487.7969 & 518.2639 & 450.2483 & 532.0331 \\
0 & 71.9995 & 74.9737 & 76.7206 & 96.6462 & 77.9037 \\
$2 \pi i$ & 470.6170 & 494.8640 & 515.6044 & 416.9562 & 527.6381 \\
$4 \pi i$ & 55.2674 & 45.3442 & 62.4615 & 109.4872 & 65.4050 \\
$6 \pi i$ & 428.2809 & 456.9464 & 466.5497 & 356.3560 & 476.1700
\end{tabular}

Table 5: Entries accurate to four decimal places

Further numerical computations show that $G_{N}( \pm 2 \pi i)$ converges as $N \gg 1$ to $\operatorname{Vol}(M) \approx 2.8281220883 \ldots$ 


\begin{tabular}{c|ccccc} 
& $-6 \pi i$ & $-4 \pi i$ & $-2 \pi i$ & 0 & $2 \pi i$ \\
\hline$G_{121}$ & 2.85352 & 0.20745 & 2.83431 & 0.15541 & 2.83431
\end{tabular}

Table 6: Entries accurate to five decimal places

\section{D The sister of the figure-eight knot complement}

This cusped manifold ( $m 003$ in SnapPea's census) is obtained by $(5,1)$ Dehn surgery on a component of the Whitehead link; see $[29 ; 36]$. The figure-eight knot complement and its sister $M$ have the smallest volume of any orientable, cusped hyperbolic 3manifold. Hyperbolic Dehn surgery on $M$ induced by $(5,1)$ and $(5,2)$ Dehn surgeries on the Whitehead link yields the Fomenko-Matveev-Weeks manifold, which has the smallest volume of any closed orientable hyperbolic 3-manifold (approximately $0.9427 \ldots)$ [20].

The Epstein-Penner decomposition of $M$ is the ideal triangulation $T$ with two tetrahedra considered in Example 2.12 and 4.11. We have already described a weak branching $\tilde{b}$ on $T$ where both tetrahedra have positive branching orientations, and computed the charges, the flattenings at positive points of the gluing variety $G(T, \tilde{b})$, and the $\mathrm{QH}$ state sums carried by $(T, \widetilde{b})$.

9D1 The $\mathbf{Q H}$ gluing variety The edge relations of $(T, \tilde{b})$ are $u_{0} u_{1}^{2} v_{0} v_{1}^{2}=1$ and $u_{0} u_{2}^{2} v_{0} v_{2}^{2}=1$. By the tetrahedral relations they are equivalent, so the gluing variety $G(T, \widetilde{b})$ is the irreducible plane curve with coordinates $\left(u_{0}, v_{0}\right)$ and defining equation $u_{0} u_{1}^{2} v_{0} v_{1}^{2}=1$. The point $\left(e^{\pi i / 3}, e^{\pi i / 3}\right)$ realizes the complete hyperbolic structure (thus $\Delta^{0}$ and $\Delta^{1}$ are regular ideal tetrahedra, as in the case of the figure-eight knot complement). There is a basis $(l, m)$ of $\pi_{1}(\partial \bar{M})$ where the dilation factors of the holonomy $\rho:=\rho\left(u_{0}, v_{0}\right)$ are given by

$$
\begin{gathered}
\operatorname{hol}_{l}(\rho)=u_{0} u_{1} v_{0}^{-1} v_{1}^{-1}=\frac{u_{0}\left(1-v_{0}\right)}{\left(1-u_{0}\right) v_{0}} \\
\operatorname{hol}_{m}(\rho)=u_{1}^{-2} v_{2}^{2}=\frac{\left(1-u_{0}\right)^{2}\left(v_{0}-1\right)^{2}}{v_{0}^{2}}
\end{gathered}
$$

The tetrahedral and edges relations between q-shape parameters are

$$
\begin{array}{rlrl}
\boldsymbol{u}_{0} \boldsymbol{u}_{1} \boldsymbol{u}_{2} & =e^{-\pi i / N}, & \boldsymbol{v}_{0} \boldsymbol{v}_{1} \boldsymbol{v}_{2} & =e^{-\pi i / N}, \\
\boldsymbol{u}_{0} \boldsymbol{u}_{1}^{2} \boldsymbol{v}_{0} \boldsymbol{v}_{1}^{2} & =e^{-2 \pi i / N}, \quad \boldsymbol{u}_{0} \boldsymbol{u}_{2}^{2} \boldsymbol{v}_{0} \boldsymbol{v}_{2}^{2}=e^{-2 \pi i / N}
\end{array}
$$

Together with the identity $\left(\boldsymbol{u}_{i+1}\right)^{N}=1 /\left(1-\left(\boldsymbol{u}_{i}\right)^{N}\right)$ and the similar one for the $\left(\boldsymbol{v}_{i}\right)^{N}$, these relations define $G_{N}(T, \widetilde{b})$. Using the tetrahedral relations it is easy to see that 
the two edge relations are equivalent. The reduced weights satisfy the compatibility relations

$$
\boldsymbol{u}_{0} \boldsymbol{u}_{1} \boldsymbol{v}_{0}^{-1} \boldsymbol{v}_{1}^{-1}=e^{k(l) / N+\pi i h(l)}, \quad \boldsymbol{u}_{1}^{-2} \boldsymbol{v}_{2}^{2}=e^{k(m) / N+\pi i h(m)} .
$$

Here we note that $H_{1}(M ; \mathbb{Z}) \cong \mathbb{Z} \oplus \mathbb{Z} / 5 \mathbb{Z}$, and that the curve $m$ or $l$ generates $H_{1}(M ; \mathbb{Z} / 2 \mathbb{Z}) \cong \mathbb{Z} / 2 \mathbb{Z}$. So the bulk weight $h$ is determined by $\kappa$, and similarly $h_{c}$ is determined by $k_{c}$. Moreover $h(m)=0 \bmod 2$, so $h$ is nonzero if and only if $h(l)=a_{0}+a_{1}+b_{0}+b_{1}=a_{2}+b_{2}=1 \bmod 2$.

At the complete hyperbolic structure we see that $\kappa(l) \in \pi i \mathbb{Z}$ and $\kappa(m) \in 2 \pi i \mathbb{Z}$, and the tetrahedral relations, the edge relations, and the compatibility relations with $\kappa$ imply the following expressions of the edge colors $a_{k}$ and $b_{k}$ in terms of $a_{1}$ and $\kappa$ :

$$
\begin{array}{rlrl}
a_{0}=\frac{\kappa(l)}{\pi i}-\frac{\kappa(m)}{2 \pi i}-2 a_{1}-2, & b_{0} & =\frac{\kappa(l)}{\pi i}-\frac{3}{2} \frac{\kappa(m)}{\pi i}-2 a_{1}-2, \\
b_{1} & =-\frac{\kappa(l)}{\pi i}+\frac{\kappa(m)}{\pi i}+a_{1}, \\
a_{2} & =a_{1}-\frac{\kappa(l)}{\pi i}+\frac{\kappa(m)}{2 \pi i}, & b_{2} & =\frac{\kappa(m)}{2 \pi i}+a_{1} .
\end{array}
$$

The same expressions are valid if one replaces $\kappa(\cdot) / \pi i$ with $(\kappa(\cdot)-\log ($ hol. $(\rho))) / \pi i$ for points $(\boldsymbol{u}, \boldsymbol{v}) \in G_{N}(T, \tilde{b})$ lying over a small neighborhood of $\left(e^{\pi i / 3}, e^{\pi i / 3}\right) \in$ $G(T, \tilde{b})$.

$9 D 2$ The state sum formulas For any $(\boldsymbol{u}, \boldsymbol{v}) \in G_{N}(T, \tilde{b})$ and any charge $c$ on $T$ we have (again we denote by $\mathcal{R}_{N}(\Delta, \boldsymbol{w})$ the tensor $\mathcal{R}_{N, *_{b}, c}\left(\boldsymbol{w}_{0}, \boldsymbol{w}_{1}\right)$ )

$$
\begin{aligned}
\mathcal{H}_{N}^{\prime}(T, \tilde{b}, c)(\boldsymbol{u}, \boldsymbol{v}) & \\
= & \sum_{i, j, k, l, I, J=0}^{N-1} \mathcal{R}_{N}\left(\Delta^{0}, \boldsymbol{u}\right)_{k, l}^{i, j} \mathcal{R}_{N}\left(\Delta^{1}, \boldsymbol{v}\right)_{j, i}^{I, J}\left(\mathcal{Q}^{2}\right)_{I}^{l} \mathcal{Q}_{J}^{k} \\
= & \left(\boldsymbol{u}_{0}^{-a_{1}^{\prime}} \boldsymbol{u}_{1}^{a_{0}^{\prime}} \boldsymbol{v}_{0}^{-b_{1}^{\prime}} \boldsymbol{v}_{1}^{b_{0}^{\prime}}\right)^{(N-1) / 2} \frac{g\left(\boldsymbol{u}_{0}\right) g\left(\boldsymbol{v}_{0}\right)}{N \phi_{N} g(1)^{2}} \\
& \quad \times \sum_{i, j, k, I=0}^{N-1} \zeta^{(i+k)(j+I)-i k+\left(j^{2}+I^{2}\right) / 2} \omega\left(\boldsymbol{u}_{0}, \boldsymbol{u}_{1}^{-1} \mid i-k\right) \omega\left(\boldsymbol{v}_{0}, \boldsymbol{v}_{1}^{-1} \mid I-j\right) .
\end{aligned}
$$

The QH state sum $\mathcal{H}_{N}^{\prime}(T, \tilde{b}, c)(\boldsymbol{u}, \boldsymbol{v})$ computes the invariant $\mathcal{H}_{N}\left(V, \rho, \kappa, k_{c}, h, h_{c}\right)$ and defines a (nonconstant) rational function on $G_{N}(T, \tilde{b})$. As for the previous examples, at a point $(\boldsymbol{u}, \boldsymbol{v})$ such that $\kappa\left(m^{p} l^{q}\right)=k_{c}\left(m^{p} l^{q}\right)=0, \mathcal{H}_{N}\left(V, \rho, \kappa, k_{c}, h, h_{c}\right)$ coincides with the $N^{\text {th }}$ quantum hyperbolic invariant of the triple $\left(M^{\prime}, L, \rho^{\prime}\right)$ obtained by hyperbolic $(p, q)$-Dehn filling of $M$. 
9D3 Some numerical results We keep the same notations as in Section 9B3 and we take again $\kappa(m)=k_{c}(m)=0$, but now both $u_{0}(A, B)$ and $v_{0}(A, B)$ have positive imaginary part, and the value of $\kappa(l)$ is given by $k_{c}(l)$ (on the left of each row), $w(A, B)$, and a flattening $f$ such that $L([w(0,0) ; f])=0$. The following table presents values of $\left|\mathcal{H}_{15}\left(V, \rho(A, B), \kappa, k_{c}, h, h_{c}\right)\right|$ up to 4 digits.

\begin{tabular}{r|rrrrr} 
& $(0,0)$ & $(1,0)$ & $(-1,0)$ & $(0,1)$ & \multicolumn{1}{c}{$(1,1)$} \\
\hline 2 & 4.7755 & 5.4995 & 4.5346 & 3.1943 & 2.6295 \\
1 & 173.2621 & 182.5736 & 173.3850 & 139.8353 & 126.3516 \\
0 & 0.2500 & 0.6231 & 0.3624 & 0.2367 & 0.1498 \\
-1 & 173.2621 & 114.3211 & 173.3850 & 214.7305 & 236.1129 \\
-2 & 4.7755 & 6.8535 & 4.5346 & 7.3011 & 9.3949
\end{tabular}

Table 7: Entries accurate to four decimal places

\section{References}

[1] H Bai, Quantum Teichmüller spaces and Kashaev's 6j-symbols, Algebr. Geom. Topol. 7 (2007) 1541-1560 MR2366170

[2] H Bai, F Bonahon, X Liu, Local representations of the quantum Teichmüller space (2007) arXiv:math.GT/0707

[3] S Baseilhac, R Benedetti, Quantum hyperbolic invariants of 3-manifolds with PSL $(2, \mathbb{C}$ )-characters, Topology 43 (2004) 1373-1423 MR2081430

[4] S Baseilhac, R Benedetti, Classical and quantum dilogarithmic invariants of flat PSL(2, C)-bundles over 3-manifolds, Geom. Topol. 9 (2005) 493-569 MR2140989

[5] S Baseilhac, R Benedetti, Quantum hyperbolic geometry, Algebr. Geom. Topol. 7 (2007) 845-917 MR2336244

[6] S Baseilhac, R Benedetti, The Kashaev and quantum hyperbolic link invariants, J. Gökova Geom. Topol. 5 (2011) 31-85 MR2872550

[7] R Benedetti, C Petronio, Lectures on hyperbolic geometry, Springer, Berlin (1992) MR1219310

[8] R Benedetti, C Petronio, Branched standard spines of 3-manifolds, Lecture Notes in Mathematics 1653, Springer, Berlin (1997) MR1470454

[9] R Benedetti, C Petronio, Spin structures on 3-manifolds via arbitrary triangulations, Algebr. Geom. Topol. 14 (2014) 1005-1054 MR3180826

[10] S Boyer, X Zhang, On Culler-Shalen seminorms and Dehn filling, Ann. of Math. 148 (1998) 737-801 MR1670053 
[11] D Bullock, Rings of $\mathrm{SL}_{2}(\mathbf{C})$-characters and the Kauffman bracket skein module, Comment. Math. Helv. 72 (1997) 521-542 MR1600138

[12] A A Champanerkar, A-polynomial and Bloch invariants of hyperbolic 3-manifolds, PhD thesis, Columbia University (2003) MR2704573 Available at http:// search.proquest.com/docview/305332823

[13] Y-E Choi, Positively oriented ideal triangulations on hyperbolic three-manifolds, Topology 43 (2004) 1345-1371 MR2081429

[14] D Cooper, M Culler, H Gillet, D D Long, P B Shalen, Plane curves associated to character varieties of 3-manifolds, Invent. Math. 118 (1994) 47-84 MR1288467

[15] F Costantino, A calculus for branched spines of 3-manifolds, Math. Z. 251 (2005) 427-442 MR2191035

[16] M Culler, P B Shalen, Varieties of group representations and splittings of 3-manifolds, Ann. of Math. 117 (1983) 109-146 MR683804

[17] N M Dunfield, Mahler's measure and the dilogarithm, II arXiv:math.NT/0308041

[18] N M Dunfield, Cyclic surgery, degrees of maps of character curves, and volume rigidity for hyperbolic manifolds, Invent. Math. 136 (1999) 623-657 MR1695208

[19] S Francaviglia, Hyperbolic volume of representations of fundamental groups of cusped 3-manifolds, Int. Math. Res. Not. 2004 (2004) 425-459 MR2040346

[20] D Gabai, R Meyerhoff, P Milley, Minimum volume cusped hyperbolic three-manifolds, J. Amer. Math. Soc. 22 (2009) 1157-1215 MR2525782

[21] A Hatcher, Algebraic topology, Cambridge Univ. Press (2002) MR1867354

[22] M Kapovich, Hyperbolic manifolds and discrete groups, Birkhäuser, Boston (2009) MR2553578

[23] R M Kashaev, Quantum dilogarithm as a 6j-symbol, Modern Phys. Lett. A 9 (1994) 3757-3768 MR1317945

[24] R M Kashaev, A link invariant from quantum dilogarithm, Modern Phys. Lett. A 10 (1995) 1409-1418 MR1341338

[25] P Kirk, E Klassen, Chern-Simons invariants of 3-manifolds decomposed along tori and the circle bundle over the representation space of $T^{2}$, Comm. Math. Phys. 153 (1993) 521-557 MR1218931

[26] S Lang, Algebraic number theory, 2nd edition, Graduate Texts in Mathematics 110, Springer, New York (1994) MR1282723

[27] F Luo, S Schleimer, S Tillmann, Geodesic ideal triangulations exist virtually, Proc. Amer. Math. Soc. 136 (2008) 2625-2630 MR2390535

[28] F Luo, S Tillmann, T Yang, Thurston's spinning construction and solutions to the hyperbolic gluing equations for closed hyperbolic 3-manifolds, Proc. Amer. Math. Soc. 141 (2013) 335-350 MR2988735 
[29] S V Matveev, A T Fomenko, Isoenergetic surfaces of Hamiltonian systems, the enumeration of three-dimensional manifolds in order of growth of their complexity, and the calculation of the volumes of closed hyperbolic manifolds, Uspekhi Mat. Nauk 43 (1988) 5-22, 247 MR937017 In Russian; translated in Russian Mathematics Surveys 43 (1988) no. 1, 3-24

[30] H Murakami, J Murakami, The colored Jones polynomials and the simplicial volume of a knot, Acta Math. 186 (2001) 85-104 MR1828373

[31] W D Neumann, Combinatorics of triangulations and the Chern-Simons invariant for hyperbolic 3-manifolds, from: “Topology '90", Ohio State Univ. Math. Res. Inst. Publ. 1, de Gruyter, Berlin (1992) 243-271 MR1184415

[32] W D Neumann, Extended Bloch group and the Cheeger-Chern-Simons class, Geom. Topol. 8 (2004) 413-474 MR2033484

[33] W D Neumann, D Zagier, Volumes of hyperbolic three-manifolds, Topology 24 (1985) 307-332 MR815482

[34] C Petronio, J Porti, Negatively oriented ideal triangulations and a proof of Thurston's hyperbolic Dehn filling theorem, Expo. Math. 18 (2000) 1-35 MR1751141

[35] P B Shalen, Representations of 3-manifold groups, from: "Handbook of geometric topology”, North-Holland, Amsterdam (2002) 955-1044 MR1886685

[36] J R Weeks, Hyperbolic structures on three-manifolds (Dehn surgery, knot, volume), $\mathrm{PhD}$ thesis, Princeton University (1985) MR2634492 Available at http:// search.proquest. com/docview/303379640

[37] T Yoshida, The $\eta$-invariant of hyperbolic 3-manifolds, Invent. Math. 81 (1985) 473514 MR807069

Institut de Mathématiques et de Modélisation, Université de Montpellier Case Courrier 51, 34095 Montpellier, Cedex 5, France

Dipartimento di Matematica, Università di Pisa Largo Bruno Pontecorvo 5, I-56127 Pisa, Italy

stephane.baseilhac@univ-montp2.fr, benedett@dm.unipi.it http://www . math. univ-montp2.fr/ baseilhac, http://www.dm.unipi.it/ benedett

Received: 1 March 2014 Revised: 25 September 2014 
